\title{
Equivariant dendroidal sets
}

\author{
Luís Alexandre Pereira
}

October 25, 2018

\begin{abstract}
We extend the Cisinski-Moerdijk-Weiss theory of $\infty$-operads to the equivariant setting to obtain a notion of $G$ - $\infty$-operads that encode "equivariant operads with norm maps" up to homotopy. At the root of this work is the identification of a suitable category of $G$-trees together with a notion of $G$-inner horns capable of encoding the compositions of norm maps.

Additionally, we follow Blumberg and Hill by constructing suitable variants associated to each of the indexing systems featured in their work.
\end{abstract}

\section{Contents}

1 Introduction 2

2 Main results 4

3 Outline 4

4 An introduction to equivariant trees 5

4.1 Planar trees . . . . . . . . . . . . . . . . . . . . . . 5

4.2 Symmetric trees . . . . . . . . . . . . . . 6

4.3 Equivariant trees . . . . . . . . . . . . . . . 8

5 Categories of trees and forests 11

5.1 Broad posets and the category of trees . . . . . . . . . 12

5.2 Categories of forests . . . . . . . . . . . . . . . . . 15

5.3 The category of equivariant trees $\ldots \ldots \ldots \ldots \ldots \ldots$

5.4 Presheaf categories . . . . . . . . . . . . . . 22

6 Normal monomorphisms and anodyne extensions 25

6.1 Equivariant normal monomorphisms . . . . . . . . . . . . 25

6.2 Equivariant anodyne extensions . . . . . . . . . . . 26

7 Tensor products 29

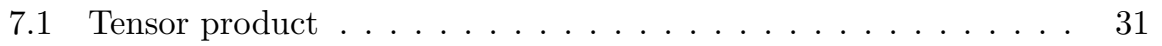

7.2 Subtrees . . . . . . . . . . . . . . . . . 32

7.3 Pushout product filtrations . . . . . . . . . . . . . . . 35

7.4 Dendroidal join . . . . . . . . . . . . . . . . . . . . 41 
8 Model structure on equivariant dendroidal sets

8.1 Existence of the model structure . . . . . . . . . . . . . . 44

8.2 Characterization of fibrant objects . . . . . . . . 46

9 Indexing system analogue results

\section{Introduction}

Operads encode a variety of algebraic structures, such as monoids, commutative monoids or (depending on the ambient category) Lie algebras, $E_{n}$-algebras, etc. Indeed, all such instances can be regarded as categories of algebras for some (fixed) suitable operad. Informally, an operad $\mathcal{O}$ consists of "sets/spaces of $n$-ary operations" $\mathcal{O}(n), n \geq 0$, each of which carries a $\Sigma_{n}$-action encoding "reordering the inputs of the operations", and a suitable notion of "composition of operations".

From the homotopy theory point of view, one of the most important classes of operads is certainly that of the $E_{\infty}$-operads, which are "up to homotopy" replacements of the commutative operad Com. More concretely, while algebras for Com are the usual commutative monoids, the algebras for an $E_{\infty}$-operad are "up to homotopy commutative monoids", where associativity and commutativity are only enforced up to homotopy. Further, $E_{\infty}$-operads $\mathcal{O}$ are characterized by the property that each space $\mathcal{O}(n)$ is a contractible space with a free $\Sigma_{n}$-action.

This work lies at the intersection of operad theory and equivariant homotopy theory. Briefly, in $G$-equivariant homotopy theory a map of $G$-spaces $X \rightarrow$ $Y$ is considered a $G$-weak equivalence only if all the induced fix point maps $X^{H} \rightarrow Y^{H}, H \leq G$ are weak equivalences. Therefore, it is no surprise that the characterization of $G$ - $E_{\infty}$-operads, i.e. $G$-equivariant operads whose algebras are " $G$-equivariant up to homotopy commutative monoids" would need to be modified. Indeed, a naive first guess might be that a $G$-operad $\mathcal{O}$ should be called $G-E_{\infty}$ if (i) each space $\mathcal{O}(n)$ has a free $\Sigma_{n}$-action and (ii) $\mathcal{O}(n)$ is $G$ contractible. Accepting this tentative characterization for the moment, such a $G$-operad is easily produced: simply taking a (non-equivariant) $E_{\infty}$-operad and giving it a trivial $G$-action yields such an example. However, it has long been known 9 that such " $G$-trivial $E_{\infty}$-operads" are not the correct replacement for the commutative operad in the equivariant setting. To see why, we consider the much studied example of $R$ a (strictly) commutative $G$-ring spectrum. For a finite $G$-set $T$ with $n$ elements it is possible to equip $R^{\wedge T} \simeq R^{\wedge n}$ with a mixed $G$-action combining the actions on $R$ and $T$. One often writes $N^{T} R$ for $R^{\wedge T}$ together with this action and calls it the Hill-Hopkins-Ravenel norm. Multiplication then induces norm maps

$$
N^{T} R \rightarrow R
$$

satisfying equivariance and associativity conditions. The flaw of " $G$-trivial $E_{\infty^{-}}$ operads" is then that they lack all norm maps (1.1) with $T$ a non-trivial $G$-set (or, after restriction to $H \leq G, T$ a non-trivial $H$-set).

In understanding this issue, note first that though $\mathcal{O}(n)$ has a $G \times \Sigma_{n}$-action when $\mathcal{O}$ is a $G$-operad, conditions (i) and (ii) above actually fail to determine a unique $G \times \Sigma_{n}$-homotopy type. Indeed, (i) implies that $\mathcal{O}(n)^{\Gamma}=\varnothing$ whenever 
$\Gamma \cap \Sigma_{n} \neq *$ while (ii) implies that $\mathcal{O}(n)^{\Gamma} \sim *$ if $\Gamma \leq G$, but these conditions leave out many subgroups $\Gamma \leq G \times \Sigma_{n}$. Indeed, there are identifications

$$
\Gamma \text { such that } \Gamma \cap \Sigma_{n}=* \leftrightarrow \text { graph of } G \geq H \rightarrow \Sigma_{n} \leftrightarrow H \text {-action on }\{1, \cdots, n\}
$$

and (ii) covers only those $\Gamma$ encoding trivial $H$-actions. The correct characterization of $G$ - $E_{\infty}$-operads is then that: (i) $\mathcal{O}(n)$ is $\Sigma_{n}$-free; (ii') $\mathcal{O}(n)$ is graph-contractible, i.e. $\mathcal{O}(n)^{\Gamma} \sim *$ for any $\Gamma \leq G \times \Sigma_{n}$ such that $\Gamma \cap \Sigma_{n}=*$.

A key observation of Blumberg and Hill in [2] is that the reason a " $G$-trivial $E_{\infty}$-operad" induces only the norm maps for trivial sets is that it satisfies (ii') only for those $\Gamma$ encoding trivial sets. Indeed, their work takes this observation much further. Motivated by the study of equivariant spectra in incomplete universes, they define a whole lattice of types of $G$-operads, which they dub $N_{\infty}$ operads, and which satisfy (ii') only for $\Gamma$ encoding $H$-sets within certain special families. Further, they call such special families indexing systems. " $G$-trivial $E_{\infty} "$ and $G-E_{\infty}$ are then the minimum and maximum types of $N_{\infty}$-operads, with the remaining types interpolating in between.

The motivation for this paper (and the larger project it belongs to) is the observation that the closure conditions for the $H$-sets in an indexing system identified in 2, Def. 3.22] admit a nice diagrammatic interpretation (discussed in 89) and that this suggests the possibility of encoding equivariant operads with norm maps (i.e. with operations in $\mathcal{O}(n)^{\Gamma}$ ) via suitable diagrammatic models.

Indeed, it is well known that composition of operations in an operad can be encoded using tree diagrams and work of Moerdijk and Weiss in [17] and follow up work of Cisinski and Moerdijk in 5 builds a category $\Omega$ of trees and a model structure on the presheaf category dSet $=\operatorname{Set}^{\Omega^{o p}}$ which is shown in the follow up papers [6] and [7] to be Quillen equivalent to the category of colored simplicial operads.

The role of this paper is to provide the equivariant analogue of the work in [17] and [5. We first identify a (non-obvious) category $\Omega_{G}$ of $G$-trees (introduced in $\$ 4.3$ and formally defined in $\$ 5.3$ ) capable of encoding norm maps and their compositions, and then adapt the proofs in $[17$ and $[5$ to prove the existence of a model structure on $\mathrm{dSet}^{G}$ whose fibrant objects, which we call $G$ - $\infty$-operads, are "up to homotopy $G$-operads with norm maps". We note that our results are not formal: indeed, while our proofs closely follow those in [17] and [5] the presence of equivariance often requires significant modifications. Moreover, we note that alternative so called "genuine" model structures on $\mathrm{dSet}^{G}$ built by formal methods (say, by mimicking the definition of the genuine model structure on Top ${ }^{G}$ ) would instead only model $G$-operads without non trivial norm maps.

\section{Acknowledgments}

The current work owes much to Peter Bonventre: the category $\Omega_{G}$ of $G$-trees, which is at the root of this paper, is a joint discovery with him; the author first heard of the notion of broad posets (due to Weiss) from him; and lastly, this work has also greatly benefited from extensive joint conversations.

I would also like to thank the anonymous referee for his or hers helpful suggestions. 


\section{$2 \quad$ Main results}

Our main result follows. It is the equivariant analogue of [5, Thm. 2.4].

Theorem 2.1. There exists a model structure on $\mathrm{dSet}^{G}$ such that

- the cofibrations are the G-normal monomorphisms;

- the fibrant objects are the $G$ - $\infty$-operads;

- the weak equivalences are the smallest class containing the $G$-inner anodyne extensions, the trivial fibrations and closed under "2-out-of-3".

Theorem 2.1 will be proven as the combination of Proposition 8.8, Theorem 8.22 and Corollary 8.23 ,

Further, letting $\mathcal{F}$ denote an indexing system (cf. [2, Def. 3.22], and as reinterpreted in Definition 9.5), we also prove the following more general result.

Theorem 2.2. For $\mathcal{F}$ a weak indexing system there exists a model structure on $\mathrm{dSet}^{G}$ such that

- the cofibrations are the $\mathcal{F}$-normal monomorphisms;

- the fibrant objects are the $\mathcal{F}$ - $\infty$-operads;

- the weak equivalences are the smallest class containing the $\mathcal{F}$-inner anodyne extensions, the $\mathcal{F}$-trivial fibrations and closed under "2-out-of-3".

Theorem 2.2 is proven at the end of $\$ 9$

Remark 2.3. In the special case where $\mathcal{F}$ is the indexing system containing only the trivial $H$-sets, the model structure given by Theorem 2.2 coincides with the "formal genuine model structure" as built using [18, Prop. 2.6] (for the collection of all subgroups $H \leq G$ ). However, this is not the case for either Theorem 2.1 or indeed the vast majority of instances of Theorem 2.2, which are not formal consequences of the existence of the model structure on dSet.

\section{Outline}

After reviewing the familiar types of trees found elsewhere in the literature (e.g. 16, 17, 5, among others), 84 provides an introductory look at the new equivariant trees that motivate this paper, focusing on examples. Most notably, each $G$-tree can be represented by two distinctly shaped tree diagrams, called the expanded and orbital representations, each capturing different key features.

$\$ 5$ lays the necessary framework for our work. Specifically, \$5.1 recalls Weiss' algebraic broad poset model [19] for the category $\Omega$ of trees, which we prefer since planar representations of $G$-trees can easily get prohibitively large. $\$ 5.2$ discusses forests, which play an auxiliary role. $\$ 5.3$ formally introduces the category $\Omega_{G}$ of $G$-trees. Lastly, \&5.4 introduces all the necessary presheaf categories, most notably the category $\mathrm{dSet}^{G}$ featured in Theorems 2.1 and 2.2.

$\$ 6$ discusses the notions of $G$-normal monomorphism and $G$ - $\infty$-operad needed to state Theorem 2.1. The former of these is straightforward, but the latter requires the key (and more subtle) notion of $G$-inner horn (Definition 6.11). 
$\$ 7$ is the technical heart of the paper, extending the key technical results 17 , Prop 9.2] and [5, Thms. 5.2 and 4.2] concerning tensor products of dendroidal sets and the dendroidal join to the equivariant setting (Theorems 7.1, 7.2, 7.4).

$\$ 8$ then finishes the proof of Theorem 2.1 by combining the results of $\$ 7$ with the arguments in the proof of the original non-equivariant result [5, Thm. 2.4].

Finally, 99 proves Theorem 2.2 by straightforward generalizations of our arguments to the framework of general indexing systems.

\section{An introduction to equivariant trees}

\subsection{Planar trees}

Operads are a tool for studying various types of algebraic structures that possess operations of several arities. More concretely, an operad $\mathcal{O}$ consists of a sequence of sets (or, more importantly to us, spaces/simplicial sets) $\mathcal{O}(n), n \geq 0$ which behave as sets (spaces/simplicial sets) of $n$-ary operations. I.e., one should have composition product maps (cf. [15, Def. 1.1] or [10, Def. 1.4])

$$
\begin{gathered}
\mathcal{O}(k) \times \mathcal{O}\left(n_{1}\right) \times \cdots \times \mathcal{O}\left(n_{k}\right) \stackrel{\circ}{\longrightarrow} \mathcal{O}\left(n_{1}+\cdots+n_{k}\right) \\
\left(\varphi, \psi_{1}, \cdots, \psi_{k}\right) \longmapsto \varphi\left(\psi_{1}, \cdots, \psi_{k}\right)
\end{gathered}
$$

and an identity $i d \in \mathcal{O}(1)$ satisfying suitable associativity and unital conditions.

A powerful tool for visualizing operadic compositions and their compatibilities is given by tree diagrams. For instance, the tree

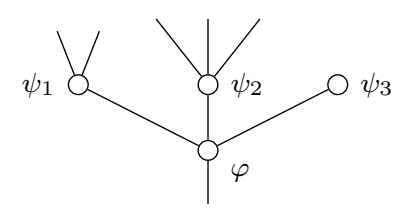

encodes the composition of operations $\varphi \in \mathcal{O}(3), \psi_{1} \in \mathcal{O}(2), \psi_{2} \in \mathcal{O}(3)$ and $\psi_{3} \in \mathcal{O}(0)$, and one has $\varphi\left(\psi_{1}, \psi_{2}, \psi_{3}\right) \in \mathcal{O}(5)$, with arity of the composite given by counting leaves (i.e. the edges at the top of the tree, not capped by a circle).

Alternatively, given the presence of the identity $i d \in \mathcal{O}(1)$, one can instead define operads using so called partial composition products [14, Def. 1.16]

$$
\begin{array}{rl}
\mathcal{O}(k) \times \mathcal{O}(n) \longrightarrow o_{i} & \mathcal{O}(n+k-1) \\
(\varphi, \psi) & \longmapsto \varphi(i d, \cdots, i d, \psi, i d, \cdots, i d)
\end{array}
$$

which are also readily visualized using trees. For example,

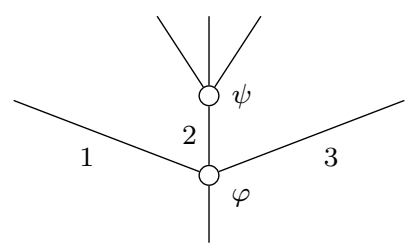


encodes the partial composition $\varphi \circ_{2} \psi=\varphi(i d, \psi, i d)$.

Heuristically, trees encoding iterations of $\circ$ are naturally tiered whereas trees encoding iterations of $\circ_{i}$ operations are not, as exemplified by the following.
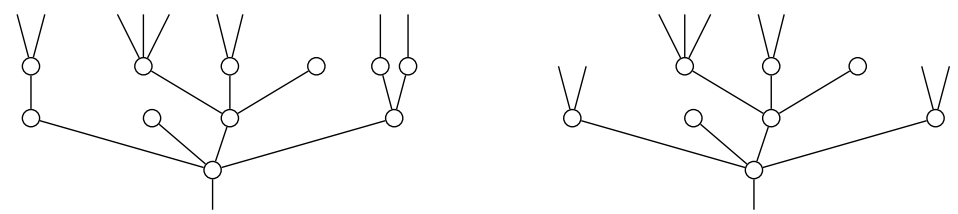

In the leftmost tree, encoding an iterated composition of $\circ$, all leaves appear at the same height and the operations, encoded by nodes (i.e. the circles) are naturally divided into levels. On the other hand, this fails for the rightmost tree, which encodes iterated compositions of $\circ_{i}$. Indeed, while the definition $\varphi \circ_{i} \psi=\varphi(i d, \cdots, i d, \psi, i d, \cdots, i d)$ would allow us to convert the rightmost tree into a tiered tree by inserting nodes labeled by $i d$, there are multiple ways to do so (indeed, the leftmost tree represents one such possibility).

In practice, the second type of trees seems to be the most convenient and we will henceforth work only with such trees.

\subsection{Symmetric trees}

The (planar) tree notation just described is suitable for working with so-called "non- $\Sigma$ operads". In many applications, however, operads possess an additional piece of structure: each set (space/simplicial set) $\mathcal{O}(n)$ has a left action of the symmetric group $\Sigma_{n}$. Heuristically, the role of this action is to "change the order of the inputs of an operation": thinking of $\varphi \in \mathcal{O}(n)$ as an operation $x_{1} \cdots, x_{n} \mapsto \varphi\left(x_{1}, \cdots, x_{n}\right)$ and letting $\sigma \in \Sigma_{n}$, then $\sigma \varphi \in \mathcal{O}(n)$ would correspond to the operation $x_{1}, \cdots, x_{n} \mapsto \varphi\left(x_{\sigma(1)}, \cdots, x_{\sigma(n)}\right)$.

When representing compositions on a (symmetric) operad, it thus becomes convenient to think of the edges above a node, which represent the inputs for the operation labeling the node, as not having a fixed order. One immediate drawback of this perspective, however, is that drawing a planar representation of such a tree on paper necessarily requires choosing an (arbitrary) order for the input edges of every node. Therefore, it is possible for different planar representations to encode the exact same information. For example, the pictures
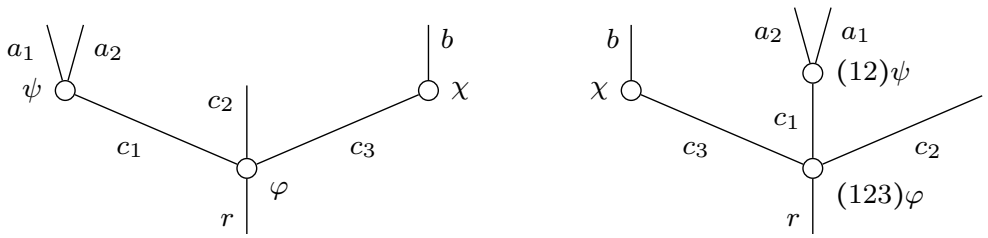

display two planar representations of the same tree that encode the same composition data. To explain why, we first point out that $a_{1}, a_{2}, b, c_{1}, c_{2}, c_{3}$ are simply the names of the edges of the tree (needed so as to distinguish different representations of the tree in the plane), by contrast with $\psi, \chi,(123) \varphi$, etc. which are operations in $\mathcal{O}$. Next, for a finite set $S$ of size $n$, denote 
$\mathcal{O}(S)=(\operatorname{Iso}(\{1, \cdots, n\}, S) \times \mathcal{O}(n))_{\Sigma_{n}}$, where the orbits $(-)_{\Sigma_{n}}$ are defined using the diagonal action, acting on the Iso $(\{1, \cdots, n\}, S)$ component by precomposition with the inverse. Note that though $\mathcal{O}(S)$ is of course isomorphic to $\mathcal{O}(n)$, the isomorphism is not canonical, depending on the choice of an isomorphism $\{1, \cdots, n\} \stackrel{\simeq}{\rightarrow} S$. With this convention, both trees in (4.3) represent the same instance of a composition

$$
\mathcal{O}\left(\left\{c_{1}, c_{2}, c_{3}\right\}\right) \times \mathcal{O}\left(\left\{a_{1}, a_{2}\right\}\right) \times \mathcal{O}(\{b\}) \stackrel{(-) \circ_{c_{1}}(-) \circ_{c_{3}}(-)}{\longrightarrow} \mathcal{O}\left(\left\{a_{1}, a_{2}, c_{2}, b\right\}\right) .
$$

The reason for the differing labels on the nodes of the trees is then that different planar representations correspond to different choices of isomorphisms $\{1, \cdots, n\} \stackrel{\sim}{\rightarrow} S$. For example, the leftmost tree uses the identification $\{1,2,3\} \simeq$ $\left\{c_{1}, c_{2}, c_{3}\right\}$ while the rightmost tree uses $\left.\{1,2,3\} \simeq\left\{c_{3}, c_{1}, c_{2}\right\}\right)$ so that, for example, the classes $\left[\left(\{1,2,3\} \stackrel{\sim}{\rightarrow}\left\{c_{1}, c_{2}, c_{3}\right\}, \varphi\right)\right],\left[\left(\{1,2,3\} \stackrel{\sim}{\rightarrow}\left\{c_{3}, c_{1}, c_{2}\right\},(123) \varphi\right)\right]$ are in fact the same element of $\mathcal{O}\left(c_{1}, c_{2}, c_{3}\right)$.

For reasons to become apparent when we discuss how to encode compositions in equivariant operads using equivariant trees, the fact that the labels in (4.3) change depending on the planar representation is rather inconvenient. Since the source of the problem is the use of non canonical isomorphisms $\mathcal{O}(S) \simeq \mathcal{O}(|S|)$, an easy solution is to use labels in $\mathcal{O}(S)$ instead. Thus, denoting by $[\psi] \epsilon$ $\mathcal{O}\left(\left\{a_{1}, a_{2}\right\}\right),[\chi] \in \mathcal{O}(\{b\}),[\varphi] \in \mathcal{O}\left(\left\{c_{1}, c_{2}, c_{3}\right\}\right)$ the classes of the operations in the leftmost tree in (4.3), the same information could then be instead encoded by labeling both trees with the common labels $[\varphi],[\psi],[\chi]$. In what follows, we refer to labels of the form $\varphi \in \mathcal{O}(n)$ as coordinate dependent labels and to $[\varphi] \in \mathcal{O}(S)$ labels as coordinate free labels.

Additionally, an important feature of symmetric trees not present in planar trees is that they have non trivial "automorphism groups". For example, the tree

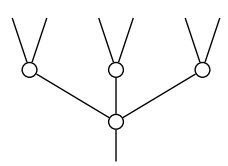

has automorphism group isomorphic to the wreath product $\Sigma_{3} 2 \Sigma_{2}=\Sigma_{3} \ltimes\left(\Sigma_{2}\right)^{\times 3}$.

Remark 4.4. An alternative and more rigorous perspective on (4.3) is provided by [16, §3], where it is explained that any tree $T$ has an associated colored operad $\Omega(T)$. Briefly, colored operads generalize operads much in the way that categories generalize monoids: each colored operad $\mathcal{O}$ has a collection of objects, morphism sets $\mathcal{O}(\underline{b} ; a)$ for an ordered tuple of source objects $\underline{b}=b_{1}, \cdots, b_{n}$ and target object $a$, units $i d_{a} \in \mathcal{O}(a ; a)$, compositions

$$
\mathcal{O}\left(b_{1}, \cdots, b_{n} ; a\right) \times \mathcal{O}\left(\underline{c}_{1} ; b_{1}\right) \times \cdots \times \mathcal{O}\left(\underline{c}_{n} ; b_{n}\right) \stackrel{\circ}{\rightarrow} \mathcal{O}\left(\underline{c}_{1}, \cdots, \underline{c}_{n} ; a\right),
$$

and isomorphisms $\mathcal{O}\left(b_{1}, \cdots, b_{n} ; a\right) \stackrel{\sigma_{*}}{\longrightarrow} \mathcal{O}\left(b_{\sigma^{-1}(1)}, \cdots, b_{\sigma^{-1}(n)} ; a\right)$ for each $\sigma \in \Sigma_{n}$ satisfying natural associativity, unitality and symmetry conditions. Note that regular operads are then "colored operads with a single color".

$\Omega(T)$ is then the colored operad with objects the set of edges of $T$ and freely generated by morphisms associated to each node. Explicitly, for the tree in (4.3) these generators are (unique) morphisms $a_{1} a_{2} \rightarrow c_{1}, b \rightarrow c_{3}$ and 
$c_{1} c_{2} c_{3} \rightarrow d$ if using the leftmost planar representation, or generators $a_{2} a_{1} \rightarrow c_{1}$, $b \rightarrow c_{3}, c_{3} c_{1} c_{2} \rightarrow d$ if using the rightmost planar representation (that the two descriptions coincide follows from the symmetry isomorphisms).

(4.3) is then a diagrammatic representation of a morphism $\Omega(T) \rightarrow \mathcal{O}$, between the colored operad $\Omega(T)$ and the regular operad $\mathcal{O}$, with the node labels being the image of the associated generators of $\Omega(T)$.

\subsection{Equivariant trees}

Throughout the following we fix a finite group $G$ and the term operad will now refer to an operad $\mathcal{O}$ together with a left $G$-action compatible will all the structure. Notably, $\mathcal{O}(n)$ now has a $G \times \Sigma_{n}$-action, so that from an equivariant homotopy theory perspective it is natural to consider fixed points $\mathcal{O}(n)^{\Gamma}$ for $\Gamma \leq G \times \Sigma_{n}$. On the other hand, since operad theory often focuses on $\Sigma$-cofibrant operads (i.e. such that $\mathcal{O}(n)$ is $\Sigma_{n}$-free), it is natural to focus attention on $\Gamma$ such that $\Gamma \cap \Sigma_{n}=*$, since for such $\mathcal{O}$ it is $\mathcal{O}(n)^{\Gamma}=\varnothing$ otherwise. The key identifications

$\Gamma$ such that $\Gamma \cap \Sigma_{n}=* \leftrightarrow$ graph of $G \geq H \rightarrow \Sigma_{n} \leftrightarrow$ action of $H \leq G$ on $\{1, \cdots, n\}$

then hint at a deep connection between $G$-operads and $H$-sets that is at the core of Blumberg and Hill's work in 2]. Briefly, to each $\Sigma$-cofibrant $G$-operad $\mathcal{O}$ they associate the family of those $\Gamma$ such that $\mathcal{O}(n)^{\Gamma} \neq \varnothing$ and, in turn, the family of the corresponding $H$-sets, $H \leq G$ [2, Def. 4.5]. They then show that such families satisfy a number of novel closure conditions [2, Lemmas 4.10, 4.11, 4.12, 4.15], and dub such a family an indexing system [2, Def. 3.22]. Moreover, analyzing their proofs one sees that the key idea is that carefully chosen fixed point conditions on the source of the composition (4.1) induce fixed point conditions on its target (for an explicit example, see (4.11) below).

The discovery of equivariant trees was the result of an attempt to encode the closure conditions of Blumberg and Hill diagrammatically, and we provide more details on how that works in \$9. For now, however, we focus on examples.

As a first guess, one might attempt to define $G$-trees simply as symmetric trees together with a $G$-action (using the automorphisms mentioned in the previous section). As it turns out, such "trees with a $G$-action" are only a part of what is required, though we will choose such trees as our first examples.

Example 4.5. Let $G=\mathbb{Z}_{/ 4}$. The following are two equivalent representations of a symmetric tree $T$ with a $G$-action.
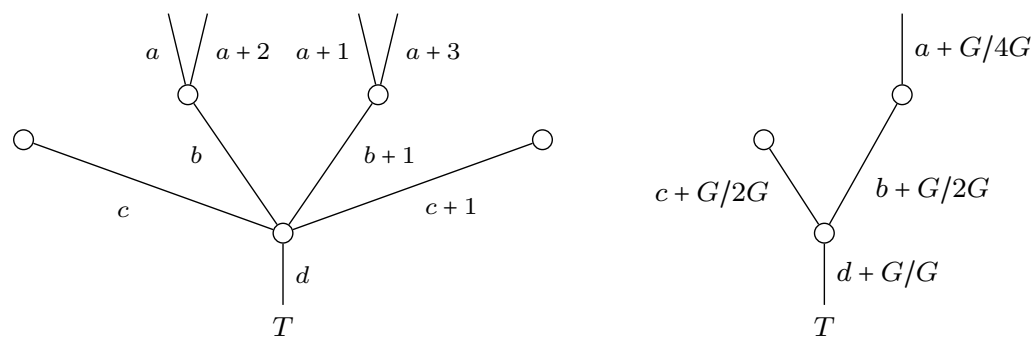

The leftmost representation, which we call the expanded representation, is simply a planar representation of the corresponding equivariant tree, together with a 
naming convention for the edges that reflects the $G$-action. More concretely, $1 \in G$ acts on the tree by sending $a$ to $a+1, a+1$ to $a+2, b$ to $b+1$, etc (note that implicitly $b+2=b, c+2=c, d+1=d)$.

The rightmost representation, which we call the orbital representation, is obtained from the expanded representation by "identifying edges which lie in the same $G$-orbit", and then labeling the corresponding "edge orbit" by the $G$-set of the edges corresponding to it.

Example 4.6. Let $G=D_{6}=\left\{e, r, r^{2}, r^{3}, r^{4}, r^{5}, s, s r, s r^{2}, s r^{3}, s r^{4}, s r^{5}\right\}$ denote the hexagonal dihedral group with generators $r, s$ such that $r^{6}=e, s^{2}=e$, srs $=r^{5}$.

Letting $H_{1} \geq H_{2} \geq H_{3}$ denote the subgroups $H_{1}=\left\langle r^{2}, s\right\rangle, H_{2}=\langle s\rangle, H_{3}=\{e\}$ one has the following representations of a tree $T$ with $G$-action.
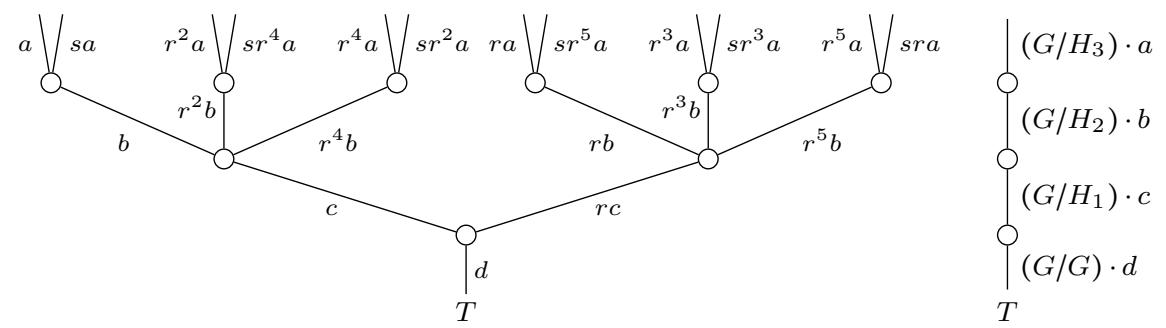

We note that it is implicit in the orbital representation that, for example, the assignment $b \mapsto c$ defines a $G$-set map $\left(G / H_{2}\right) \cdot b \rightarrow\left(G / H_{1}\right) \cdot c$ (i.e. $\left.H_{1} \geq H_{2}\right)$.

We can now ask what the analogue of the node labels in (4.2) are for such $G$-trees. For example, for $G=\mathbb{Z}_{/ 4}$ consider the label $\varphi$ in the leftmost expanded representation of the equivariant corolla (i.e. tree with a single node) below.
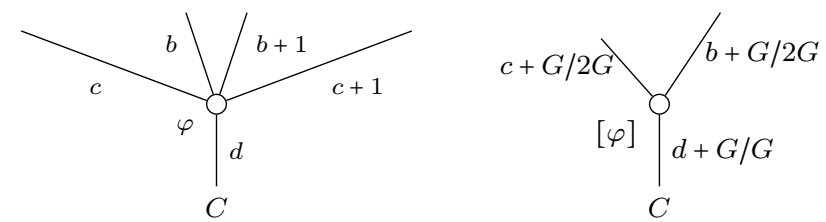

An immediate answer is provided by Remark 4.4 indeed, the corolla $C$ with a $G$-action will generate a colored operad $\Omega(C)$ with a $G$-action, so that (4.7) should encode a $G$-equivariant map $\Omega(C) \rightarrow \mathcal{O}$. Unpacking this observation, one should have $\varphi \in \mathcal{O}(4)$, but an additional equivariance condition is to be expected. To make this explicit, note first that there are left actions of both $G$ and $\Sigma_{4}$ on the set of all morphisms of $\Omega(C)$ and that these actions commute, assembling to an action of $G \times \Sigma_{4}$. As concrete examples, $1 \in \mathbb{Z}_{/ 4}$ sends the morphism $c b(b+1)(c+1) \rightarrow d$ to $(c+1)(b+1) b c \rightarrow d$ while $(124) \in \Sigma_{4}$ sends $c b(b+1)(c+1) \rightarrow d$ to $(c+1) c(b+1) b \rightarrow d$. Further, one can readily check that the $G \times \Sigma_{4}$-isotropy of the morphism $c b(b+1)(c+1) \rightarrow d$ is precisely the subgroup $\Gamma_{\{c, b, b+1, c+1\}}$ given by the graph of the homomorphism $G \rightarrow \Sigma_{4}$ encoding the $G$-set $\{c, b, b+1, c+1\}$. And since $\varphi$ is the image of that morphism, we get the sought condition $\varphi \in \mathcal{O}(4)^{\Gamma_{\{c, b, b+1, c+1\}}}$.

We now turn our attention to the orbital representation of $C$ on the right side of (4.7), which is often preferable both for conceptual reasons and compactness. 
Writing $S=\{b, c, b+1, c+1\}$, our node label is now the coordinate free label $[\varphi] \in \mathcal{O}(S)$ (indeed, a label in $\mathcal{O}(4)$ can not be used since the orbital notation provides no ordering of $S$ ). Further, $\mathcal{O}(S)$ now has two commuting $G$-actions, one induced by the structural $G$-action on $\mathcal{O}$ and one induced by the $G$-action on $S$. Referring to the combined diagonal $G$-action as the canonical $G$-action, the equivariance condition is now straightforward: it is simply $[\varphi] \in \mathcal{O}(S)^{G}$.

It seems helpful to make the equivalence between the previous two paragraphs explicit. The homomorphism $\rho: G \rightarrow \Sigma_{S}$ encoding $S$ induces a semi-direct product $G \ltimes \Sigma_{S}$ with multiplication $(g, \sigma)(\bar{g}, \bar{\sigma})=\left(g \bar{g}, \sigma \rho(g) \bar{\sigma} \rho(g)^{-1}\right) . G \ltimes \Sigma_{S}$ naturally acts on $S$ with the action of $(g, \sigma)$ given by $\sigma \rho(g)$ and on $\mathcal{O}(S)$ with action given by $\sigma g$ (where $\sigma \in \Sigma_{S}$ acts via symmetries and $g \in G$ acts via the canonical action). The isomorphism $\tau:\{c, b, b+1, c+1\} \stackrel{\simeq}{\rightarrow}\{1,2,3,4\}$ then induces an isomorphism $\tau_{\star}: G \times \Sigma_{S} \stackrel{\sim}{\rightarrow} G \times \Sigma_{4}$ via $\tau_{*}(g, \sigma)=\left(g, \tau \sigma \rho(g) \tau^{-1}\right)$. That the previous two paragraphs are equivalent is then simply the observation that $\tau_{*}$ sends the subgroup $G \leq G \ltimes \Sigma_{S}$ to $\Gamma_{\{c, b, b+1, c+1\}} \leq G \times \Sigma_{4}$, i.e. that the graph subgroup encodes the canonical action when in coordinate dependent notation.

Now that we know that corollas with $G$-actions encode operations fixed by graphs of full homomorphisms $G \rightarrow \Sigma_{n}$, we turn to the question of how to encode operations fixed by graphs of partial homomorphisms $G \geq H \rightarrow \Sigma_{n}$. A natural first guess might be that this role is played by corollas with a $\mathrm{H}$ action. However, due to the lack of full $G$-actions this would not quite provide the necessary maps for the category $\Omega_{G}$ of $G$-trees that we introduce in $\$ 5.3$. The solution is both simple and surprising: one simply "induces a tree with a $H$-action into a $G$-object". We start with an example where $H=*$.

Example 4.8. Let $G=\mathbb{Z}_{/ 3}$. The equivariant corolla $C$ with orbital representation given on the right
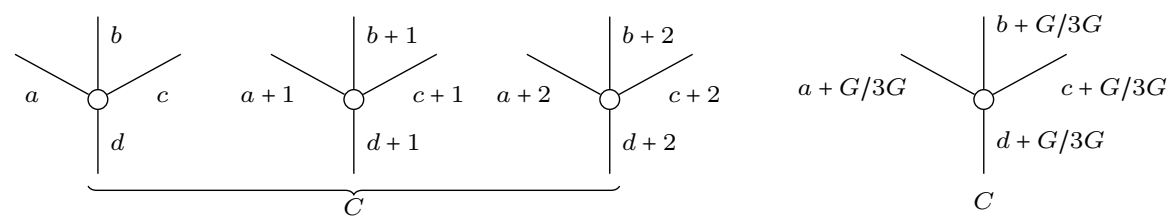

has expanded representation given by the union of the three (non-equivariant) corollas on the left. For clarity, we stress that we refer to the three trees on the left together as a forming a single $\mathbb{Z}_{/ 3}$-tree. The legitimacy of this nomenclature is born out of the role such $G$-trees play in the theory, though for now we simply point out that at least the orbital representation is a "honest" tree (for further discussion, see $\$ 5.3)$.

A map $\Omega(C) \rightarrow \mathcal{O}$ is then determined by the image of the morphism $a b c \rightarrow d$, and hence by an arbitrary operation $[\varphi] \in \mathcal{O}(\{a, b, c\}) \simeq \mathcal{O}(3)$, which determines operations $[\varphi]+1 \in \mathcal{O}(\{a+1, b+1, c+1\}),[\varphi]+2 \in \mathcal{O}(\{a+2, b+2, c+2\})$.

Example 4.9. Keeping $G=D_{6}$ and $H_{1}, H_{2}, H_{3}$ as in Example 4.6, removing 
the root orbit (i.e bottom orbit) from the $G$-tree $T$ therein yields the $D_{6}$-tree

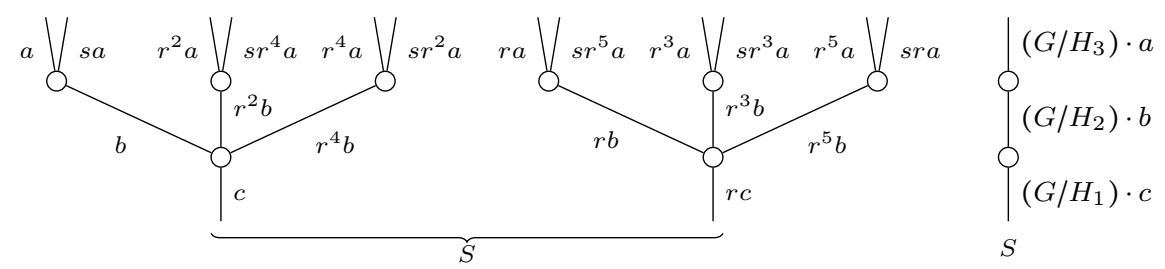

We end this introduction by illustrating the kind of compositions that $G$ trees encode. Taking the $D_{6}$-tree $S$ from Example 4.9, a map $\Omega(S) \rightarrow \mathcal{O}$ leads to node labels

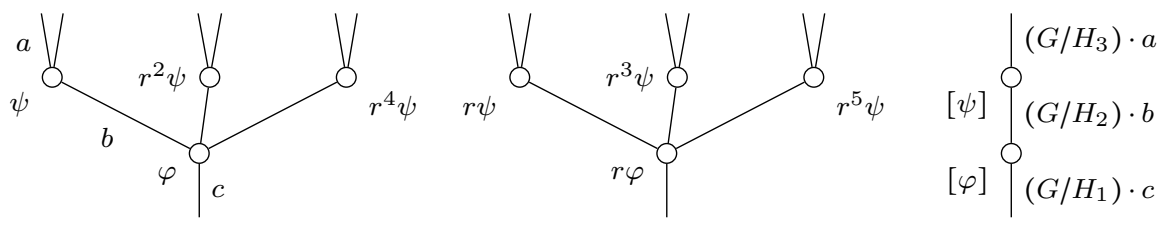

where $[\psi] \in \mathcal{O}\left(\left(H_{2} / H_{3}\right) \cdot a\right)^{H_{2}}$ and $[\varphi] \in \mathcal{O}\left(\left(H_{1} / H_{2}\right) \cdot b\right)^{H_{1}}$. We note that in particular $r[\varphi] \in \mathcal{O}\left(r\left(H_{1} / H_{2}\right) r^{-1} \cdot r b\right)^{r H_{1} r^{-1}}=\mathcal{O}\left(\left(H_{1}^{r} / H_{2}^{r}\right) \cdot r b\right)^{H_{1}^{r}}$ and likewise for $r \psi, r^{2} \psi, \cdots, r^{5} \psi$, so that we are adopting the convention that labels in the orbital notation are chosen according to the edge orbit generators $a, b, c$.

Further unpacking the map $\Omega(S) \rightarrow \mathcal{O}, S$ encodes the fact that the composition product

$$
\mathcal{O}\left(H_{1} / H_{2}\right) \times \prod_{[h] \in H_{1} / H_{2}} \mathcal{O}\left(H_{2}^{h} / H_{3}^{h}\right) \rightarrow \mathcal{O}\left(H_{1} / H_{3}\right)
$$

restricts to

$$
\mathcal{O}\left(H_{1} / H_{2}\right)^{H_{1}} \times\left(\prod_{[h] \in H_{1} / H_{2}} \mathcal{O}\left(H_{2}^{h} / H_{3}^{h}\right)^{H_{2}^{h}}\right)^{H_{1}} \rightarrow \mathcal{O}\left(H_{1} / H_{3}\right)^{H_{1}}
$$

or, using that $\left(\prod_{[h] \in H_{1} / H_{2}} \mathcal{O}\left(H_{2}^{h} / H_{3}^{h}\right)^{H_{2}^{h}}\right)^{H_{1}} \simeq \mathcal{O}\left(H_{2} / H_{3}\right)^{H_{2}}$, simply

$$
\mathcal{O}\left(H_{1} / H_{2}\right)^{H_{1}} \times \mathcal{O}\left(H_{2} / H_{3}\right)^{H_{2}} \rightarrow \mathcal{O}\left(H_{1} / H_{3}\right)^{H_{1}} .
$$

\section{$5 \quad$ Categories of trees and forests}

In this section we introduce the several categories of trees, forests and presheaves we will be working with. We will make heavy use of the broad poset framework introduced by Weiss in 19, which provides an algebraically flavored model for the category $\Omega$ of trees (cf. 16, 17, 5, 11, among others). We will find this particularly convenient since, when using tree diagrams as in $\$ 4.3$, representative examples of equivariant trees are typically quite large. 


\subsection{Broad posets and the category of trees}

We start by recalling the key notions in [19] and establishing some basic results.

Given a set $T$ we denote by $T^{+}$the free abelian monoid generated by $T$. Elements of $T^{+}$will be written in tuple notation, such as $\underline{e}=e_{1} e_{3} e_{1} e_{2}=e_{1} e_{1} e_{2} e_{3} \epsilon$ $T^{+}$for $e_{1}, e_{2}, e_{3} \in T$. We will also write $e_{i} \in \underline{e}$ whenever $e_{i}$ is a "letter" appearing in $\underline{e}, \underline{f} \subset \underline{e}$ if $\underline{f g}=\underline{e}$ for some $\underline{g} \in T^{+}$, and denote the "empty tuple" of $T^{+}$by $\epsilon$.

Definition 5.1. A (commutative) broad poset structure [19, Def. 3.2] on $T$ is a relation $\leq$ on $T^{+}, T$ (i.e. a subset of $T^{+} \times T$ ) satisfying

- Reflexivity: $e \leq e($ for $e \in T)$;

- Antisymmetry: if $e \leq f$ and $f \leq e$ then $e=f($ for $e, f \in T)$;

- Broad transitivity: if $f_{1} f_{2} \cdots f_{n}=\underline{f} \leq e$ and $\underline{g}_{i} \leq f_{i}$, then $\underline{g}_{1} \cdots \underline{g}_{n} \leq e$ (for $\left.e, f_{i} \in T, \underline{f}, \underline{g}_{i} \in T^{+}\right)$.

Remark 5.2. Omitting antisymmetry yields the notion of a pre-broad poset.

Since the main examples of broad posets are induced by constructions involving trees, we will refer to the elements of a broad poset as its edges.

Definition 5.3. A broad poset $P$ is called simple if for any broad relation $e_{1} \cdots e_{n} \leq e$ one has $e_{i}=e_{j}$ only if $i=j$.

Notation 5.4. A broad poset structure $\leq$ on $T$ naturally induces the following preorder relations on $T$ and $T^{+}$:

- for $f, e \in T$ we say that $f$ is a descendant of $e$, written $f \leq_{d} e$, if there exists a broad relation $\underline{f} \leq e$ such that $f \in \underline{f}$;

- for $\underline{f}, \underline{e} \in T^{+}$, we write $\underline{f} \leq \underline{e}$ if it is possible to write $\underline{f}=\underline{f}_{1} \cdots \underline{f} k \underline{e}=e_{1} \cdots e_{k}$ such that $\underline{f}_{i} \leq e_{i}$ for $i=1, \cdots, k$.

Remark 5.5. Generally, these preorders can be fairly counter-intuitive. For example, it is possible to have $a b \leq a$, or even both $a a \leq a$ and $a \leq a a$ simultaneously. The case of simple broad posets, however, is much simpler.

Proposition 5.6. Let $T$ be a simple broad poset. Then $\leq_{d}$ (resp. $\leq$ ) is an order relation on $T$ (resp. on $\left.T^{+}\right)$. Further, if $f_{1} \cdots f_{k} \leq e$ then the $f_{i}$ are $\leq_{d^{-}}$ incomparable (in particular, e $\underline{f} \leq e$ only if $\underline{f}=\epsilon$ ).

Proof. The "further" part is immediate: if two $f_{i}$ were $\leq_{d}$-comparable then broad transitivity would produce a non simple broad relation.

To see that $\leq_{d}$ satisfies antisymmetry, note that if $e^{\prime} f \leq e$ and $e \underline{g} \leq e^{\prime}$ then e $\underline{g f} \leq e$ so that it must be $\underline{g}=\underline{f}=\epsilon$ and the antisymmetry of $\leq$ on $T$ implies $\bar{e}=e^{\prime}$.

Finally, we show antisymmetry of $\leq$ on $T^{+}$by induction on the size of the tuple $\underline{e}$ in a pair of relations $f \leq \underline{e}$ and $\underline{e} \leq \underline{f}$. The $\underline{e}=\epsilon$ case is immediate. Otherwise let $e \in \underline{e}$ be $\leq_{d}$-maximal and choose $e \underline{g} \subset \underline{e}, f \in \underline{f}$ such that $e \underline{g} \leq f$ and choose $\underline{h} f \subset \underline{f}$ and $e^{\prime} \in \underline{e}$ such that $\underline{h} f \leq e^{\bar{T}}$. Then $e \underline{\leq}_{d} f \leq_{d} e^{\prime}$ and by $\leq_{d}$-maximality of $\bar{e}$ it must be $e=f=e^{\prime}$ and hence, by the "further" claim, also $\underline{g}=\epsilon=\underline{h}$. And since this must hold regardless of how $f, e^{\prime}, \underline{g}, \underline{h}$ are chosen, one concludes that, writing $\underline{e}=e \underline{e}^{\prime}, \underline{f}=e \underline{f}^{\prime}$ it must in fact also be $\underline{e}^{\prime} \leq \underline{f}^{\prime}$ and $\underline{f}^{\prime} \leq \underline{e}^{\prime}$, so that the induction hypothesis applies. 
Definition 5.7. An edge $e \in T$ is called

- a leaf if there are no $\underline{f} \in T^{+}$such that $\underline{f}<e$ (i.e. $\underline{f} \leq e$ and $\underline{f} \neq e$ );

- a node if there is a non empty maximum $\underline{f} \neq \epsilon$ such that $\underline{f}<e$;

- a stump if $\underline{f}=\epsilon$ is the maximum (in fact, only) $\underline{f}$ such that $\underline{f}<e$.

Further, in either the node or stump case the maximum such $\underline{f}$ is denoted $e^{\uparrow}$.

Remark 5.8. While it is customary to regard stumps simply as a type of node, we will find it convenient, in lieu of Proposition 7.15] and Lemma 7.22, to separate the two cases.

The following definition is the key purpose of [19].

Definition 5.9. A dendroidally ordered set is a finite simple broad poset $T$ satisfying the following additional conditions

- Nodal: each edge $e \in T$ is either a leaf, a node or a stump;

- Root: there is a maximum $r_{T} \in T$ for $\leq_{d}$, called the root of $T$.

Weiss proves in 19 that the category of dendroidally ordered sets (together with the obvious notion of monotonous function) is equivalent to the category $\Omega$ of trees (cf. [16, [17, [5, [11, etc). As such, we will henceforth refer to dendroidally ordered sets simply as trees and use them as our model for $\Omega$.

Example 5.10. The tree diagram

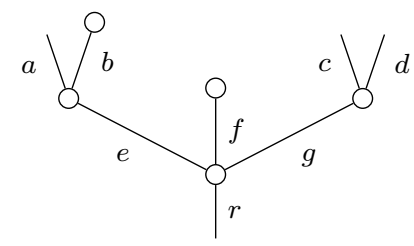

represents a broad poset structure on $\{a, b, c, d, e, f, g, r\}$. The nodes represent generating broad relations $\epsilon \leq b, a b \leq e, \epsilon \leq f, c d \leq g$, efg $\leq r$ with the other broad relations, such as afcd $\leq r$, obtained by "composition" (i.e. using broad transitivity). Note that, alternatively, one can also write $b^{\uparrow}=\epsilon, e^{\uparrow}=a b, f^{\uparrow}=\epsilon$, $g^{\uparrow}=c d, r^{\uparrow}=e f g$ to denote the generating broad relations.

We will make use of the following basic results.

Proposition 5.11. Let $T$ be a tree and $A$ any broad poset. A set map $\varphi: T \rightarrow A$ is a broad poset map if and only if $\varphi\left(e^{\uparrow}\right) \leq \varphi(e)$ for each node/stump $e \in T$.

In particular, the broad relations of $T$ are generated by the $e^{\uparrow} \leq e$ relations.

Proof. Since for any non-identity relation $\underline{f}<e$ one has $\underline{f} \leq e^{\uparrow}<e$ one can write $e^{\uparrow}=e_{1} \cdots e_{k}, \underline{f}=\underline{f}_{1} \cdots \underline{f}_{k}$ so that $\underline{f}_{i} \leq e_{i}\left(k=0\right.$ is allowed, in which case $\left.e^{\uparrow}=\underline{f}=\epsilon\right)$, so the result follows by $\leq_{d}$ induction on $e$.

The "in particular" claim follows from the identity map $i d_{T}: T \rightarrow \tilde{T}$, where $\tilde{T}$ has the broad poset structure generated by the $e^{\uparrow} \leq e$. 
Lemma 5.12. Let $T$ be a tree. For any $e \in T$ there exists a minimum $e^{\lambda} \in T^{+}$ such that $e^{\lambda} \leq e$. In fact, $e^{\lambda}=l_{1} \cdots l_{k}$ consists of those leaves $l_{i}$ such that $l_{i} \leq_{d} e$.

Further, a broad relation $f=f_{1} \cdots f_{n} \leq e$ holds if and only if $f_{i} \leq_{d} e$, the $f_{i}$ are $\leq_{d}$-incomparable and $f_{1}^{\lambda} \cdots f_{n}^{\lambda}=e^{\lambda}$.

Proof. The proof is by $\leq_{d}$ induction on $e$. The leaf case is obvious. Otherwise, let $\underline{f} \leq e^{\uparrow}<e$ be any non identity relation and write $e^{\uparrow}=e_{1} \cdots e_{k}$ and $\underline{f}=\underline{f}_{1} \cdots \underline{f}$ so that $\underline{f}_{i} \leq e_{i}$ ( $k=0$ is allowed). By induction, $e_{i}^{\lambda} \leq \underline{f}_{i} \leq e_{i}$ where $e_{i}^{\lambda}$ consists of the leaves $l$ such that $l \leq_{d} e_{i}$ and hence indeed $e^{\lambda}=e_{1}^{\lambda} \ldots e_{k}^{\lambda} \leq f$.

Only the "if" half of the "further" statement needs proof. We use the same induction argument: incomparability yields $e \notin f$ provided $f \neq e$ and, writing $e^{\uparrow}=e_{1} \cdots e_{k}$ and $f=f_{1} \cdots f_{k}$ so that $s \in \underline{f}_{i}$ if and only if $s \leq_{d} e_{i}$, the induction hypothesis applies.

Example 5.13. In Example $5.10 e^{\lambda}=a, g^{\lambda}=c d, f^{\lambda}=\epsilon, r^{\lambda}=a c d$.

We now discuss a key operation on trees: grafting. We recall that $\eta$ denotes the tree with a single edge, also denoted $\eta$, and only the identity relation $\eta \leq \eta$.

Definition 5.14. Let $T, U \in \Omega$ be trees and let $v$ denote both a leaf $v: \eta \rightarrow T$ and the root $v: \eta \rightarrow U$. The grafting $T \sqcup_{v} U$ is the pushout (of pre-broad posets)

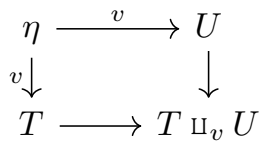

Proposition 5.15. $T \amalg_{v} U$ is a tree.

Proof. The underlying set of $T \sqcup_{v} U$ is the underlying coproduct and the broad relations are easily seen to come in three types: (i) $\underline{t} \leq t$ a relation in $T$; (ii) $\underline{u} \leq u$ a relation in $U$; (iii) $\underline{t u} \leq t$ whenever both $\underline{t} v \leq t$ in $T$ and $\underline{u} \leq v$ in $U$. The antisymmetry, simple, nodal and root conditions are straightforward.

Remark 5.16. More generally, letting $\underline{v}=v_{1} \cdots v_{n}$ denote both a tuple of leaves of $T$ and the roots $v_{i} \in U_{i}$ we similarly define a grafted tree $T \amalg_{\underline{v}}\left(U_{1} \sqcup \cdots \sqcup U_{n}\right)$. Explicitly, its broad relations have the form (i) $\underline{t} \leq t$ a relation in $T$; (ii) $\underline{u} \leq u$ a relation in some $U_{i}$; (iii) $\underline{t u}_{k_{1}} \cdots \underline{u}_{k_{p}} \leq t$ whenever $\left\{k_{i}\right\} \subset\{1, \cdots, n\}$ and both $\underline{t} v_{k_{1}} \cdots v_{k_{p}} \leq t$ in $T$ and $\underline{u}_{k_{i}} \leq v_{k_{i}}$ in $\bar{U}_{i}$.

Example 5.17. A tree representation of the grafting procedure follows.
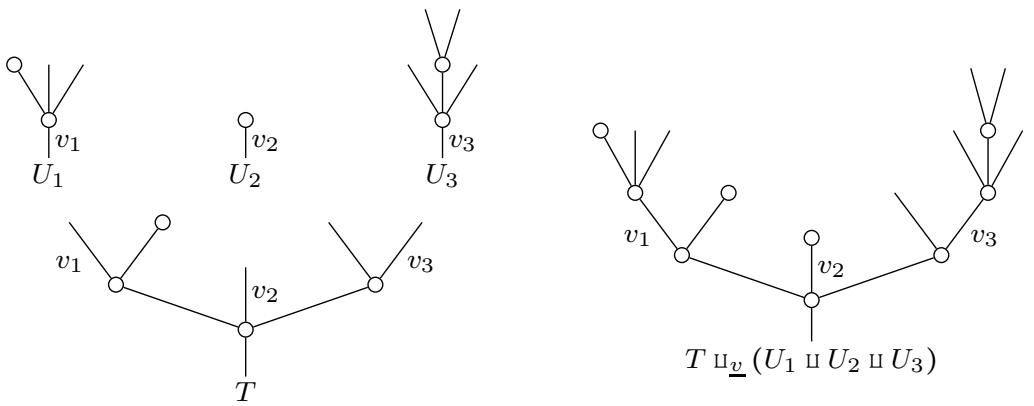
We will also find it useful to be able to reverse the grafting procedure.

For any tree $T$ and edge $e \in T$ we will let $T^{\leq e}$ denote the pre-broad poset with set $\left\{f \in T \mid f \leq_{d} e\right\}$ and the generating relations $f^{\uparrow} \leq f$ for $f \leq_{d} e$.

Similarly, for a $\leq_{d}$-incomparable tuple $\underline{e}=e_{1} \cdots e_{n}$ we will let $T_{\nless \underline{e}}$ denote the pre-broad poset with set $\left\{f \in T \mid \forall_{i} f \nless_{d} e_{i}\right\}$ and the generating relations $f^{\uparrow} \leq f$ such that $\forall_{i} f \nless_{d} e_{i}$ and $f \neq e_{i}$. Note that by incomparability it is $e_{i} \in T_{\nless \underline{e}}$.

Proposition 5.19. $T^{\leq e}$ and $T_{\nless \underline{e}}$ are trees. Hence, for any $\leq_{d}$-incomparable tuple $\underline{e}=e_{1} \cdots e_{n}$, one has

$$
T \simeq T_{\nless \underline{e} \underline{\underline{e}}} \amalg_{\underline{e}}\left(T^{\leq e_{1}} \amalg \cdots \amalg T^{\leq e_{n}}\right) .
$$

Proof. In both cases antisymmetry and simplicity are inherited from $T$. Further, since any string $f_{1} \leq_{d} f_{2} \leq_{d} \cdots \leq_{d} f_{n}$ in $T$ (note that we can assume the $\leq_{d}$ are induced by generating relations) where $f_{1}, f_{n} \in T^{\leq e}$ (resp. $f_{1}, f_{n} \in T_{\nless \underline{e}}$ ) is a string in $T^{\leq e}$ (resp. $T_{\nless \underline{e}}$ ), the nodal and root conditions also follow.

(5.20) follows from the "in particular" claim in Proposition 5.11,

Example 5.21. Denoting by $V$ the rightmost tree in (5.18), one has $U_{i}=V^{\leq v_{i}}$, $T=V_{\nless \underline{v}}$. We note here a pictorial mnemonic for our index/exponent notation: $V^{\leq v_{i}}$ denotes an "upper subtree" of $V$ while $V_{\nless \underline{v}}$ denotes a "lower subtree".

Remark 5.22. The leaves of $T_{\not \underline{e}}$ consist of the edges $e_{i} \in \underline{e}$ together with the leaves of $T$ not in $\underline{e}^{\lambda}$, i.e., those leaves $\leq_{d}$-incomparable with the $e_{i} \in \underline{e}$.

Corollary 5.23. Let $U \stackrel{\varphi}{\rightarrow} T$ be a map in $\Omega$ and let $r, \underline{l}$ be the root and leaf tuple of $U$. Then $\varphi$ naturally factors as $U \rightarrow T_{\nless \varphi(\underline{l})}^{\leq \varphi(r)} \leftrightarrow T$.

Proof. By the "in particular" claim in Proposition [5.11]it suffices to check that any relation $\underline{e} \leq^{T} e$ in $T$ is a relation in $T_{\nless e}^{\leq e}$. Since the root of $T_{\Varangle e}^{\leq e}$ is $e$ and the leaf tuple is $\underline{e}$, this follows by Lemma [5.12].

Lemma 5.24. Let $T$ be a tree and $\underline{e}$ a tuple of $\leq_{d}$-incomparable edges of $T$. Then, letting $r$ be the root of $T$, there exists a broad relation of the form $\underline{f} \leq r$.

Proof. The proof is by induction on the sum of the distances (measured in $\leq_{d}$ inequality chains) from the edges in $\underline{e}$ to the root. Clearly $r \in \underline{e}$ only if $r=\underline{e}$. Otherwise one can write $r^{\uparrow}=r_{1} \cdots r_{k}, \underline{e}=\underline{e}_{1} \cdots \underline{e}_{k}$ so that $s \in \underline{e}_{i}$ if and only if $s \leq_{d} r_{i}$. The induction hypothesis applies to $\underline{e}_{i}$ and the subtrees $T^{\leq r_{i}}$.

Since the converse of the previous lemma holds by Proposition 5.6. we obtain the following.

Corollary 5.25. If $e, f \in T$ are $\leq_{d}$-incomparable and $e^{\prime} \leq_{d} e$ then $e^{\prime}, f$ are $\leq_{d}$-incomparable.

\subsection{Categories of forests}

We will also need to discuss forests, i.e. formal coproducts of trees. We start by generalizing Definition 5.9 .

Definition 5.26. A forestially ordered set is a finite simple broad poset $F$ satisfying the following additional conditions: 
- Nodal: each edge $e \in F$ is either a leaf, a node or a stump;

- Root tuple: for each edge $e$ there is a unique $\leq_{d}$-maximal element $r \in T$ such that $e \leq_{d} r$. Further, we denote by $\underline{r}_{F}$ the tuple of $\leq_{d}$-maximal elements of $F$ and refer to it as the root tuple.

The last condition guarantees that any forestially ordered set decomposes as a disjoint union of dendroidally ordered sets, i.e. trees. We shall hence refer to these simply as forests and denote by $\Phi$ the category formed by them.

Definition 5.27. A map of forests $F \stackrel{\varphi}{\rightarrow} F^{\prime}$ is called

- wide if $\varphi\left(\underline{r}_{F}\right) \leq \underline{r}_{F}$;

- independent if there exists a tuple $\underline{e} \in F^{+}$such that $\varphi\left(\underline{r}_{F}\right) \underline{e} \leq \underline{r}_{F^{\prime}}$.

The subcategory of forests and independent (resp. wide) maps is denoted $\Phi_{w}$ (resp. $\left.\Phi_{i}\right)$. Note that there are inclusions $\Phi_{w} \hookrightarrow \Phi_{i} \hookrightarrow \Phi$.

Remark 5.28. The category $\Phi_{i}$ nearly coincides with the category of forests discussed in [11, §3.1], the only difference being that here we include the empty forest $\varnothing$.

Example 5.29. Consider the following tree $T$ and subtrees (all labels are on edges).
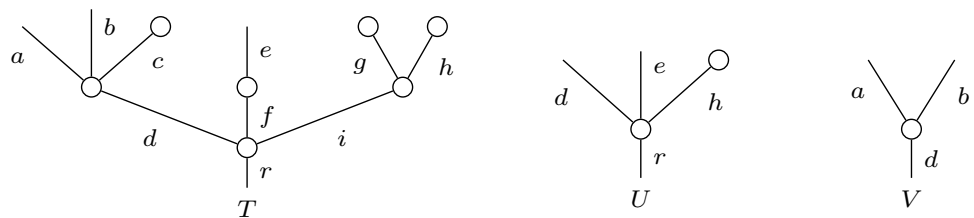

Further denoting by $\eta_{a}, \eta_{b}, \cdots$ the single edge subtrees corresponding to each edge, some examples of wide morphisms are given by the inclusions

$$
\begin{gathered}
U \hookrightarrow T, \quad V \amalg \eta_{f} \hookrightarrow T, \quad V \amalg \eta_{e} \hookrightarrow T \\
V \amalg \eta_{f} \amalg \eta_{i} \hookrightarrow T, \quad V \amalg \eta_{f} \amalg \eta_{g} \hookrightarrow T, \quad \eta_{a} \amalg \eta_{b} \amalg \eta_{f} \hookrightarrow T,
\end{gathered}
$$

some examples of non-wide independent maps are given by

$$
V \amalg \eta_{g} \hookrightarrow T, \quad \eta_{f} \amalg \eta_{i} \hookrightarrow T, \quad \eta_{g} \sqcup \eta_{h} \hookrightarrow T, \quad \varnothing \hookrightarrow T
$$

and examples of non-independent maps are given by

$$
V \amalg \eta_{r} \rightarrow T, \quad V \amalg \eta_{f} \amalg \eta_{i} \amalg \eta_{g} \rightarrow T, \quad \eta_{i} \amalg \eta_{i} \rightarrow T .
$$

The following is a useful forestial strengthening of Proposition [5.6, which follows by combining that result with Lemma 5.24.

Proposition 5.30. Let $F$ be a forest. Then, if $f \underline{f} \underline{e}=e_{1} \cdots e_{k}$ with $\leq_{d}$-incomparable $e_{i}$, the decomposition $\underline{f}=\underline{f}_{1} \cdots \underline{f}_{k}$ with $\underline{f}_{i} \leq e_{i}$ is unique. In fact, for $s \in \underline{f}$ one has $s \in \underline{f}_{i}$ iff $s \leq_{d} e_{i}$. 
Proof. If $s \leq_{d} e_{i}, s \leq_{d} e_{j}$ with $i \neq j$ the unique root $r$ such that $s \leq_{d} r$ must also satisfy $e_{i} \leq_{d} r, e_{j} \leq_{d} r$ and Lemma 5.24 can be used to produce a non simple relation. Thus such $e_{i}$ is unique and the result follows.

We now turn to describing the degeneracy-face decomposition of maps in the broad poset setting, which we obtain as Proposition 5.37 below.

Definition 5.31. A map $F \stackrel{\varphi}{\rightarrow} F^{\prime}$ in $\Phi_{i}$ is called

- a face map if the underlying set map is injective;

- a degeneracy if the underlying set map is surjective and for each leaf $l \in F$ it is $\epsilon \Varangle \varphi(l)$.

Lemma 5.32. For any map $F \stackrel{\varphi}{\rightarrow} F^{\prime}$ in $\Phi_{i}, e, \bar{e}$ are $\leq_{d}$-comparable iff $\varphi(e), \varphi(\bar{e})$ are $\leq_{d}$-comparable.

Further, if $\varphi(e)=\varphi(\bar{e})$ then $\bar{e} \underline{f} \leq e$ can hold only if $\underline{f}=\epsilon$ and thus either $e \leq \bar{e}$ or $\bar{e} \leq e$.

Proof. If $e, \bar{e}$ are not $\leq_{d}$-comparable, Lemma 5.24 ensures that there exists $\underline{f}$ such that $e \bar{e} f \leq \underline{r}_{F}$. Thus, by definition of $\Phi_{i}$, there exists $g$ such that $\bar{\varphi}(e) \varphi(\bar{e}) \varphi(f) g \underline{\leq} \varphi\left(\underline{r}_{F}\right) \underline{g} \leq \underline{r}_{F^{\prime}}$, hence $\varphi(e), \varphi(\bar{e})$ are $\leq_{d}$-incomparable.

The "further" claim is immediate.

Lemma 5.33. If $F \stackrel{\varphi}{\rightarrow} F^{\prime}$ is both a face map and a degeneracy then $\varphi$ is an isomorphism.

Proof. Bijectiveness allows us to assume that the underlying sets are the same and it thus follows from Lemma 5.32 that the relations $\leq_{d}^{F}$ and $\leq_{d}^{F^{\prime}}$ coincide. Hence, both forest structures have the same roots (i.e. $\leq_{d}$-maximal edges) and one needs only show that broad relations coincide in each of the constituent trees. But noting that a leaf $l$ is precisely a $\leq_{d}$-minimal edge such that $\epsilon \nless l$, the definition of degeneracy implies that $F, F^{\prime}$ have the same leaves and the result follows by the criterion in the "further" part of Lemma 5.12

Lemma 5.34. Let $F \stackrel{\varphi}{\rightarrow} F^{\prime}$ be a map in $\Phi_{i}$ and $f \leq e$ be a broad relation in $F$ such that $\varphi(\underline{f}) \neq \varphi(e)$. Then for any $\underline{f}^{\prime}, e^{\prime}$ such that $\varphi\left(\underline{f}^{\prime}\right)=\varphi(\underline{f}), \varphi\left(e^{\prime}\right)=\varphi(e)$ it is also $\underline{f}^{\prime} \leq e^{\prime}$.

Proof. Writing $\underline{f}=f_{1} \cdots f_{n}, \underline{f}^{\prime}=f_{1}^{\prime} \cdots f_{n}^{\prime}$, the condition $\varphi(\underline{f}) \neq \varphi(e)$ ensures $f_{i}<_{d}$ $e$, and thus Lemma 5.32 implies it is also $f_{i}^{\prime}<_{d} e^{\prime}$. Using the characterization in Lemma 5.12, the desired relation $\underline{f}^{\prime} \leq e^{\prime}$ will hold provided we show that $a^{\lambda}=\bar{a}^{\lambda}$ whenever $\varphi(a)=\varphi(\bar{a})$. But since leaves are $\leq_{d}$-minimal, this too follows from Lemma 5.32

Remark 5.35. It follows from the "further" part in Lemma 5.32 together with antisymmetry that the pre-image of any edge by a map $\varphi$ always consists of a linearly ordered subset of edges. As such, degeneracies are necessarily maps that "collapse linear sections of a tree", and indeed that was their original description 
in 19. A typical degeneracy (sending edges labeled $a_{i}, b_{i}, c_{i}, d_{i}$ to respective edges $a, b, c, d)$ is pictured below.
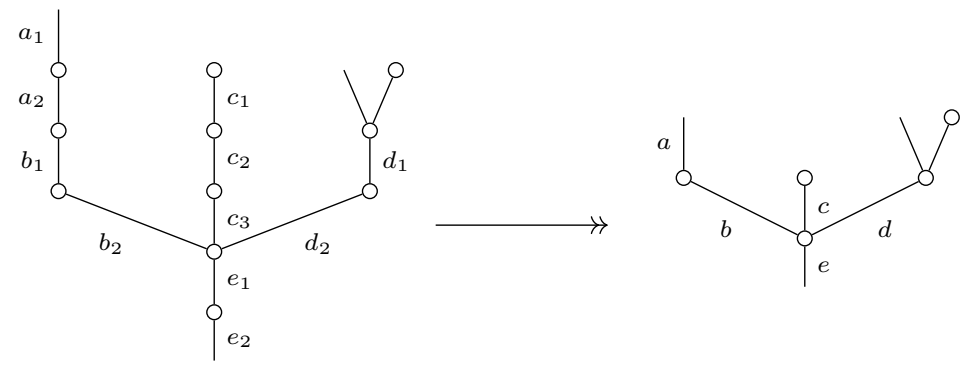

Lemma 5.36. Let $\varphi: F \rightarrow F^{\prime}$ be a map in $\Phi_{i}$ and let $U \subset F$ be any subbroad poset consisting of exactly one edge in each pre-image of $\varphi$ and the broad relations of $F$ between them. Then $U$ is a forest.

Proof. Simplicity of $U$ is inherited from $F$. Given $u \in U$, let $\bar{u} \in F$ be the $\leq_{d^{-}}$ minimal edge such that $\varphi(\bar{u})=\varphi(u)$. Lemma 5.34 implies that $u$ is a leaf in $U$ if $\bar{u}$ is a leaf in $F$. Otherwise, $\bar{u}^{\uparrow}<\bar{u}$ (in $F$ ) and again by Lemma 5.34 the unique tuple $\underline{v}$ of $U$ such that $\varphi(\underline{v})=\varphi\left(\bar{u}^{\uparrow}\right)$ provides the desired node tuple $u^{\uparrow}=\underline{v}$ for $U$. Lastly, by Lemma 5.34 $s$ will be a root of $U$ iff $\varphi(s)=\varphi(r)$ for $r$ a root of F.

We now prove the following factorization result, which in the $\Omega$ case first appeared as [16, Lemma 3.1] and in the $\Phi_{i}$ case was proven in [11, Lemma 3.1.3].

Proposition 5.37. Each map $\varphi$ of $\Phi_{i}$ has a factorization $\varphi=\varphi^{+} \circ \varphi^{-}$as a degeneracy followed by a face. Further, this decomposition is unique up to unique isomorphism.

Finally, the decomposition restricts to the subcategories $\Omega$ and $\Phi_{w}$.

Proof. Given a map $F \stackrel{\varphi}{\rightarrow} F^{\prime}$ and picking any $U$ as in Lemma 5.36, the isomorphism $U \simeq \varphi(F)$ allows us to equip $\varphi(F)$ with a forest structure. Moreover, by Lemma 5.34 the broad relations of $\varphi(F)$ are exactly the image of those in $F$, and thus independent of the chosen $U$. The existence of a factorization follows.

The uniqueness of the $F \stackrel{\varphi^{-}}{\rightarrow} G \stackrel{\varphi^{+}}{\rightarrow} F^{\prime}$ factorization follows since by the description of the broad structure on $\varphi(F)$ there is clearly a broad poset map $\varphi(F) \rightarrow G$, which by Lemma 5.33 is an isomorphism.

Further, the decomposition restricts to $\Omega$ since the image by a degeneracy map of a tree is necessarily a tree and restricts to $\Phi_{w}$ since any edge surjective map $F \stackrel{\varphi^{-}}{\rightarrow} G$ must map roots to roots and hence it must be $\varphi^{-}\left(\underline{r}_{F}\right)=\underline{r}_{G}$.

Corollary 5.38. If $F \stackrel{\rho}{\rightarrow} F^{\prime}$ is a degeneracy in any of $\Omega, \Phi_{i}, \Phi_{w}$ then the broad relations in $F^{\prime}$ are precisely the image of the broad relations of $F$. Further, any section $F^{\prime} \stackrel{s}{\rightarrow} F$ of the underlying set map is a section in $\Omega, \Phi_{i}, \Phi_{w}$.

The following will be needed in $\$ 5.4$ when discussing dendroidal boundaries. 
Corollary 5.39. In any of $\Omega, \Phi_{i}, \Phi_{w}$, pairs of degeneracies with common domain have absolute pushout 1 .

Proof. We will throughout write $\Xi$ for any of $\Omega, \Phi_{i}, \Phi_{w}$ and $\Xi[F] \in$ Set $^{\Xi^{o p}}$ for the presheaf represented by $F \in \Xi$. Given a diagram of degeneracies $E \stackrel{\bar{\rho}}{\longleftarrow} F \stackrel{\rho}{\rightarrow} E_{0}$ we first inductively extend it to a diagram of degeneracies as on the left below
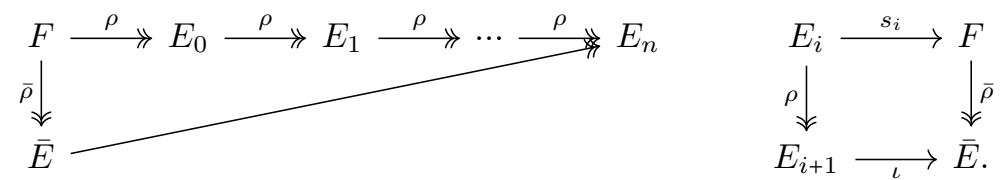

as follows: assuming $E_{0} \rightarrow \cdots \rightarrow E_{i}$ have been built, Corollary 5.38 implies that a (necessarily unique) compatible degeneracy $\bar{E} \rightarrow E_{i}$ exists iff it exists at the level of the underlying sets; otherwise, there must exist edges $e_{1}, e_{2} \in E_{i}$ and lifts $f_{1}, f_{2} \in F$ such that $\rho^{\circ i+1}\left(f_{k}\right)=e_{k}$ and $\bar{\rho}\left(f_{1}\right)=\bar{\rho}\left(f_{2}\right)$, and choosing $s_{i}$ to be a section of $\rho^{\circ i+1}$ such that $s_{i}\left(e_{k}\right)=f_{k}$, one defines $\rho: E_{i} \rightarrow E_{i+1}$ via the degeneracy-face factorization of $\bar{\rho} s_{i}$, as in the right square in (5.40). The condition on $s_{i}$ then guarantees that $\rho: E_{i} \rightarrow E_{i+1}$ is never an isomorphism, so that this procedure always terminates.

It remains to show that $\Xi[\bar{E}] \amalg_{\Xi[F]} \Xi\left[E_{0}\right] \rightarrow \Xi\left[E_{n}\right]$ is an isomorphism. Surjectivity is immediate from the existence of a section $s: E_{n} \rightarrow E_{0}$ of $\rho^{\circ n}$. For injectivity, the existence of a section $\bar{s}: \bar{E} \rightarrow F$ of $\bar{\rho}$ implies that one can represent any element of $\Xi[\bar{E}] \amalg_{\Xi[F]} \Xi\left[E_{0}\right]$ by an element in $\Xi\left[E_{0}\right]$, i.e. as an equivalence class $[\varphi]$ for a map $\varphi: T \rightarrow E_{0}$. It now suffices to show by induction on $i$ that if $\rho^{\circ i} \varphi=\rho^{\circ i} \psi$ then it is $[\varphi]=[\psi]$ or, equivalently, that it is $[\varphi]=\left[\rho s_{i} \rho^{\circ i} \varphi\right]$. The case $i=0$ is trivial. Otherwise, we claim that

$$
[\varphi]=\left[\rho s_{i-1} \rho^{\circ i-1} \varphi\right]=\left[\rho s_{i-1} \rho^{\circ i} s_{i} \rho^{\circ i} \varphi\right]=\left[\rho s_{i} \rho^{\circ i} \varphi\right]
$$

The leftmost and rightmost identities follow from the induction hypothesis for $i-1$ and the functions $\varphi, \rho s_{i} \rho^{\circ i} \varphi$. For the inner identity, one considers the lifts $s_{i-1} \rho^{\circ i-1} \varphi: T \rightarrow F, s_{i-1} \rho^{\circ i} s_{i} \rho^{\circ i} \varphi: T \rightarrow F$ and notes that postcomposition with $\bar{\rho}$ yields $\bar{\rho} s_{i-1} \rho^{\circ i-1} \varphi=\iota \rho^{\circ i} \varphi$ and $\bar{\rho} s_{i-1} \rho^{\circ i} s_{i} \rho^{\circ i} \varphi=\iota \rho^{\circ i+1} s_{i} \rho^{\circ i} \varphi=\iota \rho^{\circ i} \varphi$. Injectivity now follows, finishing the proof.

We now recall the usual $([19,[17,[5],[11)$ description of faces as composites of maximal "codimension 1 " faces. We first discuss some terminology.

Firstly, we will regard a face $F^{\prime}$ of $F$ as a subset of $F$ together with a subset of the broad relations of $F$, and write $F^{\prime} \hookrightarrow F$ to indicate this. Further, if the broad relations between edges of $F^{\prime}$ in the broad posets $F^{\prime}$ and $F$ coincide, then we will call $F^{\prime}$ a full face of $F$ and write $F^{\prime} \subset F$ instead.

Secondly, an edge $e \in F$ is called external if $e$ is either a leaf or a root and internal otherwise.

Finally, we denote a generating broad relation of $F$ by $v_{e}=\left(e^{\uparrow} \leq e\right)$ and refer to it as the vertex at $e$.

Notation 5.41. The maximal faces of $F$ in $\Omega, \Phi_{i}, \Phi_{w}$ have the following types.

\footnotetext{
${ }^{1}$ Recall that an absolute colimit can be described as either a colimit that is preserved by the Yoneda embedding or (equivalently) a colimit that is preserved by any functor.
} 
- The inner face (valid for any of $\Omega, \Phi_{i}, \Phi_{w}$ ) associated to an inner edge $e$ is the full face $F-e \subset F$ obtained by removing $e$;

- The leaf vertex outer face (valid for any of $\Omega, \Phi_{i}, \Phi_{w}$ ) associated to a vertex $v_{e}$ such that $e^{\uparrow}$ consists of leaves is the full face $F_{\nless e} \subset F$ obtained by removing the leafs in $e^{\uparrow}$;

- The stump outer face (valid for any of $\Omega, \Phi_{i}, \Phi_{w}$ ) associated to a stump vertex $v_{e}=\left(\epsilon=e^{\uparrow} \leq e\right)$ is the face $F_{\nless e} \hookrightarrow F$ with the same edges as $F$ but removing $\epsilon \leq e$ as a generating broad relation (note that this also removes some composite relations);

- The root vertex outer face (valid only for $\Omega$ ) for an edge $e \in r^{\uparrow}$ such that the edges of $r^{\uparrow}$ other than $e$ are leaves is the full face $T^{\leq e} \subset T$ consisting of those edges $\bar{e}$ such that $\bar{e} \leq_{d} e$;

- The root face (valid only for $\Phi_{i}, \Phi_{w}$ ) associated to a root $r_{i} \in \underline{r}_{F}$ which is not also a leaf is the full face $F-r_{i} \subset F$ obtained by removing $r_{i}$;

- The stick component face (valid only for $\Phi_{i}$ ) associated to a stick $\eta \in F$ (i.e. an edge that is simultaneously a root and a leaf) is the full face $F-\eta \subset F$ obtained by removing $\eta$.

Remark 5.42. The implicit claim that an inner face $F-e$ is itself a forest can easily be checked using Lemma 5.12, which shows that $f^{\uparrow, F-e}$ can be defined to consist of the $\leq_{d}$-maximal edges $f_{i} \neq e$ such that $f_{i} \leq_{d} f$.

Similarly, Lemma 5.12 shows that a broad relation $f_{1} \cdots f_{n} \leq f$ in $F$ holds in a stump outer face $F_{\nless e}$ if the condition $e \leq_{d} f$ implies that $e \leq_{d} f_{i}$ for some $i$.

Example 5.43. Consider the trees in Example 5.29, One can write

$$
U=\left(\left(\left(\left(\left(T_{\nless c}\right)_{\nless d}\right)-f\right)-i\right)-g\right),
$$

where the intermediate steps (from the inside out) are a stump face, a leaf vertex face and three inner faces. Further, both $\eta_{a}=V^{\leq a}$ and $\eta_{b}=V^{\leq b}$ are root vertex outer faces of $V$ when viewing $V$ as a tree and $\eta_{a} \amalg \eta_{b}=V-d$ is a root face of $V$ when viewing $V$ as a forest.

\subsection{The category of equivariant trees}

Let $G$ be a finite group. We will denote by $\Phi^{G}$ the category of $G$-forests, i.e. forests equipped with a $G$-action.

Definition 5.44. The category of $G$-trees, denoted $\Omega_{G}$, is the full subcategory $\Omega_{G} \subset \Phi^{G}$ of $G$-forests $F$ such that the root tuple $\underline{r}_{F}$ consists of a single $G$-orbit.

Remark 5.45. The relationship between $\Phi^{G}$ and $\Omega_{G}$ is similar to the relationship between the category $\mathrm{Fin}^{G}$ of finite $G$-sets and the orbital category $\mathrm{O}_{G}$ consisting of the orbital $G$-sets $G / H$.

Examples of equivariant trees can be found throughout 4.3 , The author is aware that the fact that $G$-trees often "look life forests" is likely counterintuitive at first (indeed, that was a major hurdle in the development of the theory presented in this paper). However, the following two facts may assuage 
such concerns: (i) similarly to how a non-equivariant tree is a forest that can not be decomposed as a coproduct of forests, so too a $G$-tree can not be equivariantly decomposed as a coproduct of $G$-forests; (ii) the orbital representation of a $G$ tree (cf. \$4.3) always does "look like a tree".

Remark 5.46. Note that $\Omega_{G}$, the category of $G$-trees, is rather different from $\Omega^{G}$, the category of trees with a $G$-action. In fact, each $G$-tree is (non-canonically) isomorphic to a forest of the form $G \cdot{ }_{H} T$ for some $H \leq G$ and $T \in \Omega^{H}$. More precisely, one has the following elementary proposition.

Proposition 5.47. $\Omega_{G}$ is equivalent to the Grothendieck construction for the functor (where $\mathrm{O}_{G}$ is the orbit category; see Remark 5.45)

$$
\begin{aligned}
& \mathrm{O}_{G}^{o p} \longrightarrow \text { Cat } \\
& G / H \longmapsto \Omega \underline{G / H}
\end{aligned}
$$

where $G / H$ denotes the groupoid with objects the cosets $g H$ and arrows $g H \stackrel{\bar{g}}{\rightarrow}$ $\bar{g} g H$.

Remark 5.48. There is a natural inclusion $G \times \Omega \hookrightarrow \Omega_{G}$ given by regarding each object $(*, T) \in G \times \Omega$ as the $G$-tree given by the $G$-free forest $G \cdot T$.

Remark 5.49. While maps in $\Omega$ can be built out of two types of maps, faces and degeneracies (Proposition 5.37), in $\Omega_{G}$ we need a third type of map: quotients.

To see this, note that by Proposition 5.47, each $G$-tree $T$ sits (up to equivalence) inside one of the subcategories $\Omega \frac{G / H}{}$, and that $\Omega^{H}$ is equivalent to the latter. Since it is immediate by (the proof of) Proposition 5.37 that the degeneracy-face decomposition extends to $\Omega^{H}$, Proposition 5.47 implies that any map in $\Omega_{G}$ factors as a degeneracy followed by a face (both inside one of

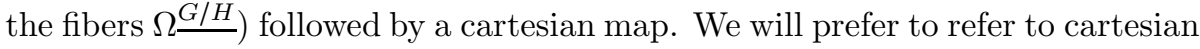
maps as quotients.

For a representative example, let $G=\mathbb{Z}_{/ 8}$ and consider the map below (represented in orbital notation, cf. \$4.3), where we follow the following conventions: (i) edges in different trees with the same label are mapped to each other; (ii) if an edge is denoted $e^{+i}$, we assume that its orbit is disjoint from that of $e$ and that, if no edge labeled $e^{+i}$ appears in the target tree, then $\varphi\left(e^{+i}\right)=\varphi(e)+i$.

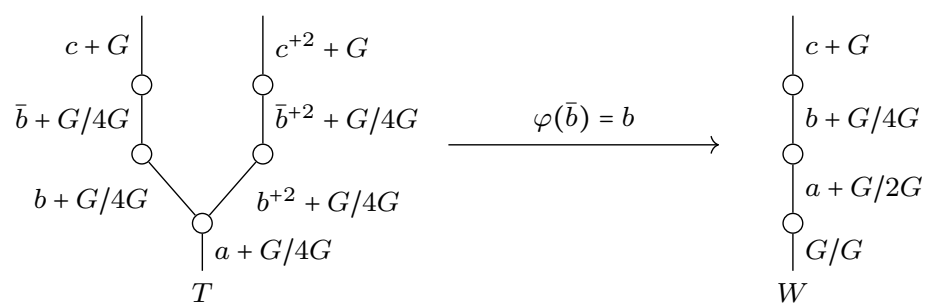

This map can be factored as (where, for the sake of brevity, we write $a+G / 4 G$ as $a / 4$, etc.) 


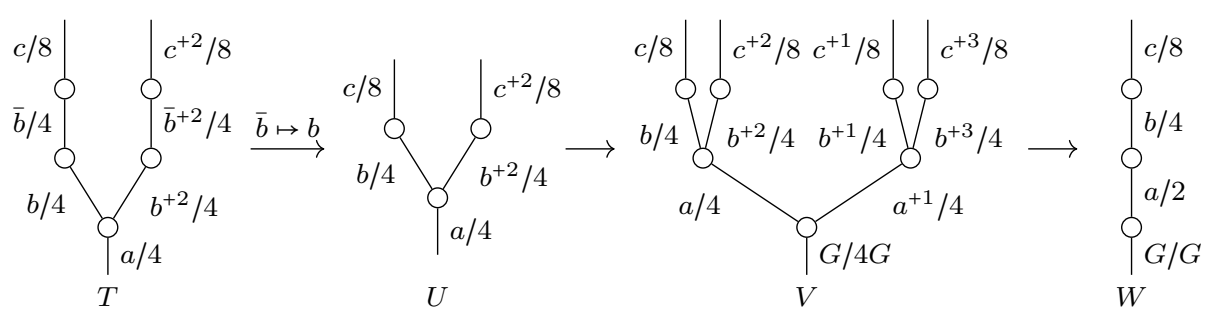

It is perhaps worthwhile to unpack the last map in (5.50), which is an example of a quotient. The $G$-tree labeled $V$ can be written as $V \simeq G \cdot{ }_{4 G} \bar{V}$ where $\bar{V} \in \Omega^{4 G}$ is the tree with a $4 G$-action pictured below.

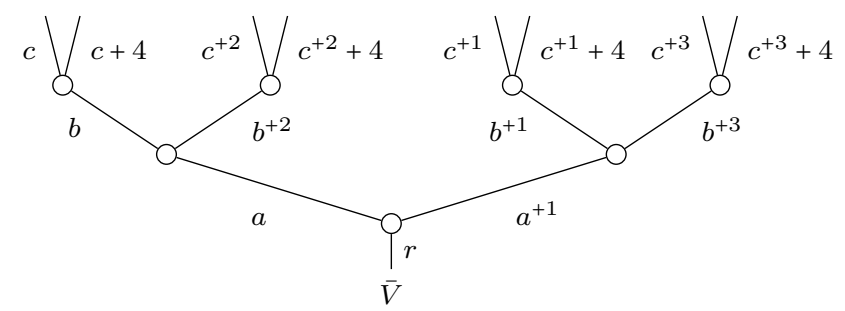

In words, $V$ consists (non-equivariantly) of four trees identical to $\bar{V}$ which are interchanged by the action of elements of $G$ other than 0,4 . W, on the other hand, consists of a single (non-equivariant) tree, also shaped like $\bar{V}$, and can be thought of as the quotient of $V$ obtained by gluing the four trees so that the edges $e^{+i}+j$ and $e+i+j$ are identified.

Remark 5.51. One particularly convenient property of $\Omega$ is that $\Omega^{o p}$ is a generalized Reedy category, in the sense of [1]. In fact, $\Omega$ is a dualizable generalized Reedy category, so that both $\Omega$ and $\Omega^{o p}$ are generalized Reedy.

Unfortunately, this is not the case for $\Omega_{G}$ : while indeed $\Omega_{G}$ itself can be shown to be generalized Reedy, the opposite category $\Omega_{G}^{o p}$ is not. The problem is readily apparent in the factorization in (5.50). Indeed, for the Reedy factorizations to hold (cf. [1, Defn. 1.1(iii)]), quotient maps would need to be considered the same type of maps as face maps, i.e. degree raising maps of $\Omega_{G}$. However, the quotient map in (5.50) fails [1, Defn. 1.1(iv')], since there is an automorphism of $V$ (given by $e^{+i} \mapsto e^{+i+1}-1$, where $e^{+0}$ is interpreted as $e$, and yet undefined $e^{+i}$ labels are interpreted by regarding $i \in \mathbb{Z}_{/ k}$ as needed) compatible with the quotient map to $W$.

\subsection{Presheaf categories}

We now establish some key terminology and notation concerning the presheaf categories we will use. Recall that the category of dendroidal sets is the presheaf category dSet $=\operatorname{Set}^{\Omega^{o p}}$.

Definition 5.52. The category of G-equivariant dendroidal sets is the category

$$
\mathrm{dSet}^{G}=\operatorname{Set}^{\Omega^{o p} \times G} .
$$


The category of genuine G-equivariant dendroidal sets is the category

$$
\mathrm{dSet}_{G}=\operatorname{Set}^{\Omega_{G}^{o p}} .
$$

Twisting the inclusion in Remark 5.48 by the inverse map $G^{o p} \stackrel{(-)^{-1}}{\longrightarrow} G$ yields an inclusion $u: \Omega^{o p} \times G \hookrightarrow \Omega_{G}$.

Proposition 5.53. The adjunction

$$
u^{*}: \mathrm{dSet}_{G} \rightleftarrows \mathrm{dSet}^{G}: u_{*}
$$

identifies $\mathrm{dSet}^{G}$ as a reflexive subcategory of $\mathrm{dSet}_{G}$.

Remark 5.55. Since Theorem 2.1 concerns $\mathrm{dSet}^{G}$, that category will be our main focus throughout the present paper, although $\mathrm{dSet}_{G}$ also plays a role in its proof (cf. 88.2).

Nonetheless, $\mathrm{dSet}_{G}$ is arguably the most interesting category. Indeed, the adjunction (5.54) bears many similarities to the adjunction sSet ${ }_{G}^{\mathrm{O}_{G}} \rightleftarrows \mathrm{sSet}^{G^{o p}}$ and, as will be shown in upcoming work, the full structure of the "homotopy operad" of a $G$ - $\infty$-operad is described as an object in $\operatorname{dSet}_{G}$ rather than in $\mathrm{dSet}^{G}$ (more precisely, the claim is that the homotopy operad of a $G$ - $\infty$-operad forms a "colored genuine equivariant operad"; (single colored) genuine equivariant operads have been recently formalized by the author and Peter Bonventre in [3). We note that this is similar to how $\pi_{n}$ of a $G$-space forms a $G$-coefficient system rather than just a $G$-set. We conjecture that a model structure on $\operatorname{dSet}_{G}$ making (5.54) into a Quillen equivalence exists, and that too is the subject of current work. The presence of extra technical difficulties when dealing with $\Omega_{G}$ (cf. Remark 5.51), however, make it preferable to address the $\mathrm{dSet}^{G}$ case first.

Notation 5.56. Recall the usual notation

$$
\Omega \stackrel{T \mapsto \Omega[T]}{\longrightarrow} \mathrm{dSet}
$$

for the Yoneda embedding.

One can naturally extend this notation to the category $\Phi$ of forests: given $F=\amalg_{i} T_{i}$, set $\Omega[F]=\amalg_{i} \Omega\left[T_{i}\right]$. Passing to the $G$-equivariant object categories and using the inclusion $\Omega_{G} \rightarrow \Phi^{G}$ we will slightly abuse notation and write

$$
\Omega_{G} \stackrel{T \mapsto \Omega[T]}{\longrightarrow} \mathrm{dSet}^{G} .
$$

More explicitly, if $T \simeq G \cdot{ }_{H} T_{e}$ for some $T_{e} \in \Omega^{H}$, then $\Omega[T] \simeq G \cdot{ }_{H} \Omega\left[T_{e}\right]$, where $\Omega\left[T_{e}\right]$ is just the Yoneda embedding of (5.57) together with the resulting $H$-action.

Remark 5.59. Note that while (5.58) defines "representable functors" for each $T \in \Omega_{G}$, given a presheaf $X \in \mathrm{dSet}^{G}$ the evaluations $X(U)$ are defined only for $U \in \Omega$, i.e., for $U$ a non-equivariant tree.

This is in contrast with $\mathrm{dSet}_{G}$, where both representables and evaluations are defined in terms of $\Omega_{G}$. We note that to reconcile this observation with the inclusion $u_{*}$ of (5.54) the non-equivariant tree $U \in \Omega$ should be reinterpreted as the free $G$-tree $G \cdot U \in \Omega_{G}(\mathrm{cf}$. Remark [5.48). 
We end this section by discussing a category of "forestial sets" which, while secondary for our purposes, will greatly streamline our discussion of the dendroidal join in $\$ 7.4$

Definition 5.60. The category of wide forestial sets is the category

$$
\text { fSet }_{w}=\operatorname{Set}^{\Phi_{w}^{o p}} .
$$

Remark 5.61. The category $\mathrm{fSet}_{i}=\operatorname{Set}^{\Phi_{i}^{o p}}$ of what we might call "independent forestial sets" was one of the main objects of study in [11, where they are called simply "forest sets".

Mimicking (5.57) by writing $\Phi_{i}[F] \in \mathrm{fSet}_{i}$ for the representable functor of $F \in \Phi_{i}$, it is shown in [1] that one can define a formal boundary $\partial \Phi_{i}[F]$ possessing the usual properties one might expect.

We will find it desirable to be able to use the analogous construction for the representable $\Phi_{w}[F] \in \mathrm{fSet}_{w}$, but this does not quite follow from the result in [11, since while $\Phi_{i}[F] \in \mathrm{fSet}_{i}$ can be forgotten to a presheaf $u^{*} \Phi_{i}[F] \in \mathrm{fSet}_{w}$, one typically has a proper inclusion $\Phi_{w}[F] \hookrightarrow u^{*} \Phi_{i}[F]$.

We thus instead mimic the discussion in [1], making use of the key technical results established in 5.2

Letting $\Xi$ denote any of $\Omega, \Phi_{i}, \Phi_{w}$ and setting

$$
|F|=\#\{\text { edges of } F\}+\#\{\text { stumps of } F\},
$$

then Lemma 5.33 and Proposition 5.37 say that $\Xi$ is a dualizable generalized Reedy category (cf. 1, Defn. 1.1]). As in [1, §6], call an element $x: \Xi[F] \rightarrow X$ of a presheaf $X \in \mathrm{Set}^{\Xi^{o p}}$ degenerate if it factors through a non invertible degeneracy operator and non-degenerate otherwise. Corollary 5.39 then allows us to adapt the proof of [1, Prop. 6.9] to obtain the following.

Proposition 5.62. Let $X \in \operatorname{Set}^{\Xi^{o p}}$ for $\Xi$ any of $\Omega, \Phi_{i}, \Phi_{w}$. Then any element $x: \Xi[F] \rightarrow X$ has a factorization, unique up to unique isomorphism,

$$
\Xi[F] \stackrel{\rho_{x}}{\longrightarrow} \Xi[G] \stackrel{\bar{x}}{\rightarrow} X
$$

as a degeneracy operator $\rho_{x}$ followed by a non degenerate element $\bar{x}$.

Defining skeleta as in [1, §6] the proof of [1, Cor. 6.8] yields the following.

Corollary 5.63. Let $\Xi$ be any of $\Omega, \Phi_{i}, \Phi_{w}$. The counit $s k_{n} X \rightarrow X$ for $X \in$ Set $^{\Xi^{o p}}$ is a monomorphism whose image consists of those elements of $X$ that factor through some $\Xi[F] \rightarrow X$ for $|F| \leq n$.

Definition 5.64. Let $\Xi$ be any of $\Omega, \Phi_{i}, \Phi_{w}$. The formal boundary

$$
\partial \Xi[F] \hookrightarrow \Xi[F]
$$

is the subobject formed by those maps that factor through a non invertible map in $\Xi^{+}$, i.e. through a non invertible face map.

Note that by combining the Reedy axioms [1, Defn. 1.1] with Corollary [5.63 one has

$$
\partial \Xi[F] \simeq s k_{|F|-1} \Xi[F] .
$$




\section{Normal monomorphisms and anodyne exten- sions}

\subsection{Equivariant normal monomorphisms}

Recalling that the cofibrations in dSet are not the full class of monomorphisms [5. Prop. 1.5], but rather the subclass of so called normal monomorphisms, one should expect a similar phenomenon to take place in $\mathrm{dSet}^{G}$.

We start by noting that for $X \in \mathrm{dSet}^{G}$ and $U \in \Omega$, the set $X_{U}=X(U)$ is acted on by the group $G \times \Sigma_{U}$, where $\Sigma_{U}$ denotes the automorphism group of $U$.

Definition 6.1. A subgroup $N \leq G \times \Sigma_{U}$ is called a $G$-graph subgroup if $N \cap \Sigma_{U}=$ *.

It is straightforward to check that a $G$-graph subgroup $N$ can equivalently be described by a partial homomorphism $G \geq H \stackrel{\rho}{\rightarrow} \Sigma_{U}$. Further, since such a $\rho$ allows us to write $U \in \Omega^{H}$, one has that each $G$-graph subgroup $N$ has an associated $G$-tree $G \cdot{ }_{H} U$. More precisely, one has the following result.

Proposition 6.2. The functor $\Omega[-]: \Omega_{G} \rightarrow \mathrm{dSet}^{G}$ induces an equivalence between $\Omega_{G}$ and the full subcategory of quotients of the form $(G \cdot \Omega[U]) / N$ for $U \in \Omega$ and $N \leq G \times \Sigma_{U}$ a $G$-graph subgroup.

Recalling the discussion following (5.57) one can, for a forest $F \simeq \amalg_{i} T_{i}$ in $\Phi$, define $\partial \Omega[F]=\sqcup_{i} \partial \Omega\left[T_{i}\right]$. Carrying this discussion through to $G$-objects leads to the following definition.

Definition 6.3. The boundary inclusions of $\mathrm{dSet}^{G}$ are the maps of the form

$$
\partial \Omega[T] \hookrightarrow \Omega[T]
$$

for $T \in \Omega_{G}$.

More explicitly, if $T \simeq G \cdot H T_{e}$ for some $T_{e} \in \Omega^{H}$, then the (non-equivariant) presheaf $\Omega\left[T_{e}\right]$ inherits a $H$-action and (6.4) is isomorphic to the map

$$
G \cdot{ }_{H}\left(\partial \Omega\left[T_{e}\right] \hookrightarrow \Omega\left[T_{e}\right]\right)
$$

or, letting $N \leq G \times \Sigma_{T_{e}}$ denote the $G$-graph subgroup associated to $T_{e}$,

$$
\left(G \cdot\left(\partial \Omega\left[T_{e}\right] \hookrightarrow \Omega\left[T_{e}\right]\right)\right) / N .
$$

The following is an immediate generalization of [1, Prop. 7.2].

Proposition 6.5. Let $\phi: X \rightarrow Y$ be a map in $\mathrm{dSet}^{G}$. Then the following are equivalent.

(i) for each tree $U \in \Omega$, the relative latching map $l_{U}(\phi): X_{U} \underset{L_{U} X}{\amalg} L_{U} Y \rightarrow Y_{U}$ is a $\Sigma_{U}$-free extension;

(ii) $\phi$ is a monomorphism and, for each $U \in \Omega$ and non degenerate $y \in Y_{U}-$ $\phi(X)_{U}$, the isotropy group $\left\{g \in G \times \Sigma_{U} \mid g y=y\right\}$ is a $G$-graph subgroup; 
(iii) for each $n \geq 0$, the relative $n$-skeleton $s k_{n}(\phi)=X \underset{s k_{n} X}{\amalg} s k_{n} Y$ is obtained from the relative $(n-1)$-skeleton by attaching boundary inclusions.

Definition 6.6. A monomorphism satisfying any of the equivalent conditions in Proposition 6.5 will be called a G-normal monomorphism.

Remark 6.7. Note that by Proposition 6.5(i) a monomorphism in $\mathrm{dSet}^{G}$ is $G$-normal iff it is normal as a map in dSet [1, Prop. 7.2]. As such, we often drop $G$ from the terminology. Moreover, all monomorphisms over a $G$-normal dendroidal set are hence $G$-normal monomorphisms [5, Corollaries 1.7 and 1.8].

\subsection{Equivariant anodyne extensions}

The key to the preceding section was the observation that if $T \in \Omega^{H}$ then the usual boundary $\partial \Omega[T]$ inherits a $H$-action. However, such is not the case for inner horns: if $e \in T$ is an inner edge, then $\Lambda^{e}[T]$ (cf. [17, §5]) will inherit a $H$-action iff $e$ is a $H$-fixed edge.

Therefore, to define $G$-inner horns, one must treat all inner edges in an inner edge orbit in an uniform way. To do so, we first recall the notion of generalized inner horns (cf. [17, Lemma 5.1]).

Definition 6.8. Let $E \subset \operatorname{Inn}(T)$ be a subset of the inner edges of $T \in \Omega$. We define

$$
\Lambda^{E}[T] \hookrightarrow \partial \Omega[T] \hookrightarrow \Omega[T]
$$

to be the subpresheaf formed by the union of those faces other than the inner faces of the form $T-E^{\prime}$ for $E^{\prime} \subset E$.

More generally, given a forest $F=\amalg_{i} T_{i}$ and $E=\amalg_{i} E_{i}$ with $E_{i} \subset \operatorname{Inn}\left(T_{i}\right)$ we set

$$
\Lambda^{E}[F]=\amalg_{i} \Lambda^{E_{i}}\left[T_{i}\right] .
$$

Remark 6.10. The reader of [11 may note that (6.9) clashes with [11, §3.6]. This is because in [11] the presheaf being defined lives in $\mathrm{fSet}_{i}$ rather than in dSet.

Definition 6.11. The generating G-inner horn inclusions are the maps in $\mathrm{dSet}^{G}$ of the form

$$
\Lambda^{G e}[T] \rightarrow \Omega[T]
$$

where $T \in \Omega_{G}$ is a $G$-tree and $G e$ is the $G$-orbit of an inner edge $e$.

Definition 6.12. A $G$-dendroidal set $X$ is called a $G$ - $\infty$-operad if $X$ has the right lifting property with respect to all generating $G$-inner horn inclusions.

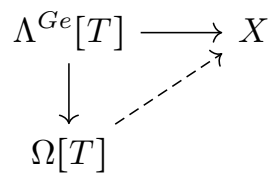

Further, $A \rightarrow B$ is called a $G$-inner anodyne extension if it is in the saturation of the generating $G$-inner horn inclusions under pushouts, transfinite compositions and retracts. 
Example 6.13. If one considers the $G=\mathbb{Z}_{/ 4}$-tree $T$ in Example 4.5 , one possible inner orbit edge is $G b=\{b, b+1\}$. The following are the (inner) faces of $T$ not included in $\Lambda^{G e}[T]$.

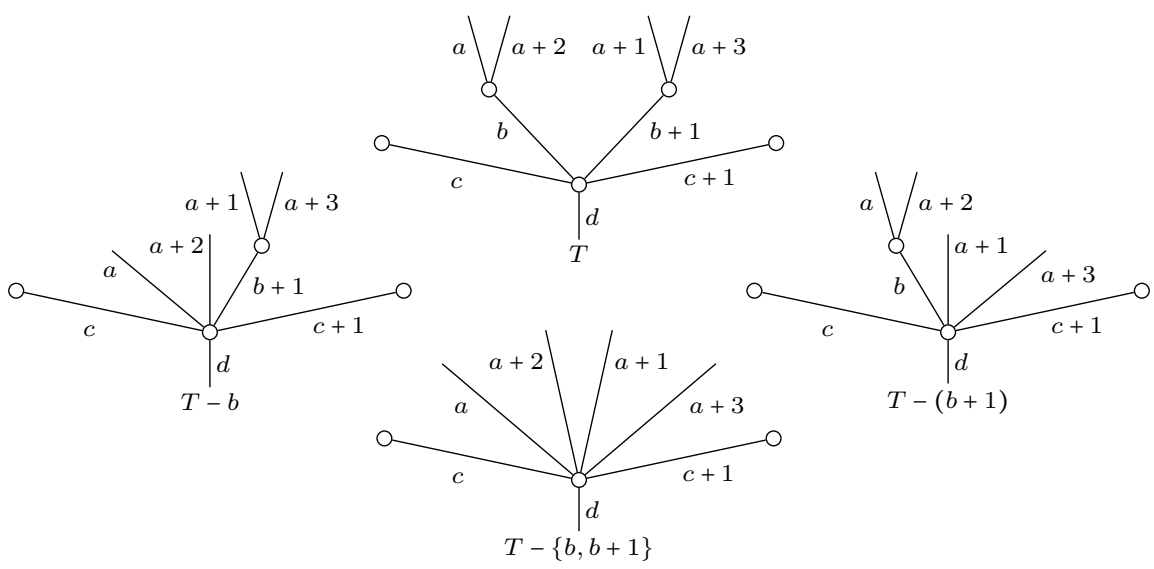

We recall (cf. Remark 5.59) that since presheaves $X \in \mathrm{dSet}^{G}$ are only evaluated on non-equivariant trees $U \in \Omega$, the faces above are merely non-equivariant faces of the equivariant tree $T$ : indeed, $T-b$ and $T-(b+1)$ do not admit a full compatible $G$-action. Rather, $G$ acts instead on the set of such faces and since $T-b$ admits a compatible $H=2 G$-action and one can think of the disjoint union $(T-b) \amalg(T-(b+1))$ as the $G$-tree $G \cdot H(T-b)$.

Remark 6.14. An eventual goal of the project this work belongs to is to show that there is a Quillen equivalence

$$
W_{!}: \mathrm{dSet}^{G} \rightleftarrows \mathrm{sOp}^{G}: h c N_{d},
$$

generalizing [7, Thm. 8.15] (where sOp ${ }^{G}$ is the category of $G$-equivariant colored simplicial operads and $h c N_{d}$ is the dendroidal homotopy coherent nerve).

While the proof of such a result is work in progress, and some of the best evidence in that direction is the subject of a current parallel write-up making Remark 5.55 precise, we provide here a first piece of evidence by generalizing [17, Thm. 7.1].

Proposition 6.15. Suppose that $\mathcal{O} \in \mathrm{sOp}^{G}$ is locally $G$-graph fibrant, i.e., that $\mathcal{O}(\underline{a} ; b)^{\Gamma}$ is fibrant whenever $\Gamma \leq G \times \Sigma_{a}$ stabilizes $\underline{a}, b$ and satisfies $\Gamma \cap \Sigma_{a}=*$.

Then $h c N_{d}(\mathcal{O})$ is a $G$ - $\infty$-operad.

Proof. Since any $G$-tree has the form $G \cdot{ }_{H} T$ for some $T \in \Omega^{H}$, it suffices after unpacking adjunctions to solve all the lifting problems as on the left below.

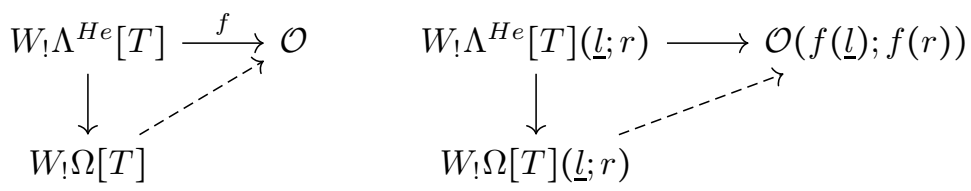

Repeating the argument in the proof of [17, Thm. 7.1], it suffices to build this lift for the mapping spaces between the leaves $\underline{l}$ and root $r$ of $T$. I.e., one needs only 
solve the rightmost lifting problem in (6.16), which needs only be equivariant with respect to the subgroup $\Gamma \leq H \times \Sigma_{l}$ encoding the $H$-set $\underline{l}$ (crucially, note that operadic compatibility is automatic). Since the condition on $\mathcal{O}$ guarantees that $\mathcal{O}(f(\underline{l}) ; f(r))$ is genuinely $\Gamma$-fibrant and the vertical map on the right side of (6.16) is (generalizing the formula in the proof of [7, Prop. 4.5])

$$
(\{1\} \rightarrow \Delta[1])^{\square H e} \square(\partial \Delta[1] \rightarrow \Delta[1])^{\square I n n(T)-H e},
$$

which is a genuine $\Gamma$-trivial cofibration, the result follows.

We will develop for $G$-inner horns most of the key results of [17] and [5, the proofs of which turn out to need only moderate modifications in order to generalize to the equivariant context.

The hardest of those results, concerning the tensor product, will be the subject of $\$ 7$. To finish this section, we collect a couple of easier results, starting with the analogue of [17, Lemma 5.1].

Proposition 6.17. Let $T \in \Omega_{G}$ be a $G$-equivariant tree and $E$ a $G$-equivariant subset of the inner edges of $T$. Then the generalized $G$-horn inclusion

$$
\Lambda^{E}[T] \rightarrow \Omega[T]
$$

is $G$-inner anodyne.

Proof. Since $E$ consists of a union of edge orbits, one immediately reduces to proving that maps of the form

$$
\Lambda^{E}[T] \rightarrow \Lambda^{E-G e}[T]
$$

are $G$-inner anodyne. In the non-equivariant case [17, Lemma 5.1] such maps can be described as single pushouts, but here we require multiple pushouts, naturally indexed by an equivariant poset which we now describe.

Firstly, let $T_{e}$ denote the (non-equivariant) tree component containing the edge $e$ and set $H \leq G$ to be its isotropy, resulting in a canonical identification $G \cdot H T_{e} \simeq T$. Writing $\operatorname{Inn}_{H e}\left(T_{e}\right)$ for the $H$-poset (under inclusion) of the inner faces of $T_{e}$ collapsing only edges in $H e$, it suffices to check that, for any $H$ equivariant convex subsets $B \subset B^{\prime} \subset \operatorname{lnn}_{H e}\left(T_{e}\right)$ it is

$$
\Lambda^{E}[T] \cup \bigcup_{g \in G, U \in B} \Omega[g U] \rightarrow \Lambda^{E}[T] \cup \bigcup_{g \in G, U \in B^{\prime}} \Omega[g U]
$$

$G$-inner anodyne. Without loss of generality, we may assume $B^{\prime}$ is obtained from $B$ by adding a single orbit $H V$ and, setting $\bar{H} \leq H$ to be the isotropy of $V$ in $\operatorname{lnn}_{H e}\left(T_{e}\right)$, we claim that (6.18) is a pushout of

$$
G \cdot{ }_{\bar{H}}\left(\Lambda^{E_{e}-H e}[V] \rightarrow \Omega[V]\right),
$$

where $E_{e}=E \cap T_{e}$ denotes the subset of inner edges of $T_{e}$ that are in $E$. This claim is straightforward except for the following: one needs to note that the $G$ isotropy of any faces in $\operatorname{lnn}_{E_{e}-H e}(V)$ (i.e. those faces missing from $\Lambda^{E_{e}-H e}[V]$ ) is indeed contained in $\bar{H}$, and this follows since $\bar{H}$ can also be described as the subgroup of $G$ sending the edge subset $H e \cap V=G e \cap V$ to itself.

This concludes the proof by nested induction on the order of $G$ and the number of $G$-orbits of $E$.

\footnotetext{
${ }^{2}$ Recall that a subset $B \subset \mathcal{P}$ of a poset $\mathcal{P}$ is called convex if $\bar{b} \leq b$ and $b \in B$ implies $\bar{b} \in B$.
} 
The following is the equivariant analogue of [17, Lemma 5.2]. Note that edge orbits of a $G$-tree $T$ are encoded by maps $G / H \cdot \eta \stackrel{G / H \cdot e}{\longrightarrow} T$ for some $H \leq G$.

Proposition 6.19. Suppose that $T$ has a leaf orbit and $U$ root orbit both isomorphic to $G / H$. Write $V=T \amalg_{G / H \cdot \eta} U$ for the grafted $G$-tree.

Then

$$
\Omega[T] \amalg_{\Omega[G / H \cdot \eta]} \Omega[U] \rightarrow \Omega[V]
$$

is inner G-anodyne.

Proof. Let Out $(V)$ denote the $G$-poset of outer faces (which have the form $V_{\nless \underline{e}}^{\leq e}$, and are hence not inner faces of any other face) of the grafted tree $V$, and Out $_{\phi T, U}(V)$ the $G$-subposet of those outer faces contained in neither $T$ nor $U$.

It suffices to show that for all $G$-equivariant convex subsets $B \subset B^{\prime}$ of Out $_{\phi T, U}(V)$ it is

$$
\Omega[T] \cup \Omega[U] \cup \bigcup_{R \in B} R \rightarrow \Omega[T] \cup \Omega[U] \cup \bigcup_{R \in B^{\prime}} R
$$

$G$-inner anodyne (indeed, (6.21) recovers (6.20) when $B=\varnothing, B^{\prime}=$ Out $_{\phi T, U}(V)$ ).

As before, we can assume $B^{\prime}$ is obtained from $B$ by adding the orbit $G S$ of a single outer face $S$. Letting $H \leq G$ denote the isotropy of $S$, one has that (6.21) is then the pushout (note that the $G$-isotropy of an inner face of the outer face $S$ is at most $H$ ) of

$$
G \cdot H\left(\Lambda^{\operatorname{Inn}(S)}[S] \rightarrow \Omega[S]\right),
$$

finishing the proof.

Remark 6.22. A key difference between the proofs of Propositions 6.17 and 6.19 versus their non-equivariant analogues is the need to check that the isotropies are correct when attaching equivariant horns.

\section{Tensor products}

Our goal in this section is to prove equivariant analogues of [17, Prop. 9.2], [5. Thm. 5.2] and [5, Thm. 4.2], which are the key technical results in their respective papers. These results concern the interaction of anodyne extensions with the tensor product and the join constructions, which we recall in $\$ 7.1$ and $\$ 7.4$. We now present our versions of the results, starting with the analogue of [17. Prop. 9.2].

Theorem 7.1. Let $S, T \in \Omega_{G}$ be $G$-trees and let $G \xi$ be an inner orbit edge of T. Then the map

$$
\partial \Omega[S] \otimes \Omega[T] \coprod_{\partial \Omega[S] \otimes \Lambda^{G \xi}[T]} \Omega[S] \otimes \Lambda^{G \xi}[T] \rightarrow \Omega[S] \otimes \Omega[T]
$$

is a G-inner anodyne extension if either

(i) both $S$ and $T$ are open $G$-trees (i.e. have no stumps);

(ii) at least one of $S, T$ is a linear $G$-tree (i.e. isomorphic to $G \cdot_{H}[n]$ for $[n] \in \Delta)$. 
The proof of Theorem 7.1 will be the subject of $\$ 7.3$. More specifically, the result will follow from Proposition 7.44 when $B=\varnothing$ and $B^{\prime}=\operatorname{IE}_{G \xi}(S \otimes T)$.

The following is the equivariant analogue of [5, Thm. 5.2].

Theorem 7.2. Let $S \in \Omega^{G}$ be a tree with a $G$-action such that $S \neq \eta$ and denote by $A \rightarrow \Omega[S] \otimes \Omega[1]$ the pushout product map

$$
\partial \Omega[S] \otimes \Omega[1] \coprod_{\partial \Omega[S] \otimes\{1\}} \Omega[S] \otimes\{1\} \rightarrow \Omega[S] \otimes \Omega[1]
$$

Then there is a factorization $A \rightarrow B \rightarrow \Omega[S] \otimes \Omega[1]$ such that

(a) $A \rightarrow B$ is a $G$-inner anodyne extension;

(b) there is a pushout (the join $S \star \eta$ is introduced in Definition 7.46 )

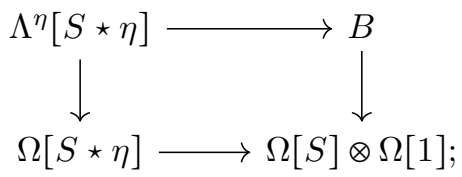

(c) letting $\eta \stackrel{r}{\rightarrow} S$ denote the root edge, the composite

$$
\Omega[1] \simeq \Omega[\eta \star \eta] \stackrel{r \star i d}{\longrightarrow} \Omega[S \star \eta] \rightarrow \Omega[S] \otimes \Omega[1]
$$

coincides with the composite

$$
\Omega[1] \simeq \eta \otimes \Omega[1] \stackrel{r \otimes i d}{\longrightarrow} \Omega[S] \otimes \Omega[1]
$$

Theorem 7.2 will be proven at the end of $\$ 7.3$ as a direct consequence of the arguments used in the proof of Proposition 7.44. We note that $\Lambda^{\eta}[S \star \eta]$ is an outer horn, lacking only the outer face $(S \star \eta)-\eta=(S \star \eta)^{\leq r} \simeq S$.

The following is the equivariant analogue of [5, Thm. 4.2]. Note that we use the notation $i: \Delta \rightarrow \Omega$ for the inclusion and $i^{*}: \mathrm{d}$ Set $\rightarrow \mathrm{s}$ Set for the restriction.

Theorem 7.4. Let $S \in \Omega^{G}$ be a tree with a G-action. Assume further that $S$ has at least two vertices and unary root vertex $G / G \cdot \Omega[1] \stackrel{v_{r}}{\longrightarrow} S$. Then a lift exists in any commutative diagram

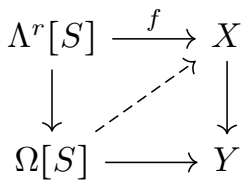

such that $X \rightarrow Y$ is a $G$-inner fibration between $G$ - $\infty$-operads and $f\left(v_{r}\right)$ is an equivalence in the $\infty$-category $i^{*}\left(X^{G}\right)$.

We will prove Theorem 7.4 at the end of $\$ 7.4$ 


\subsection{Tensor product}

To keep the proofs of Theorems 7.1 and 7.2 compact we will prefer to use broad poset language throughout. We start by defining tensor products in this framework.

Given $\underline{s} \in S^{+}, \underline{t} \in T^{+}$, we will let $\underline{s} \times \underline{t} \in(S \times T)^{+}$denote the obvious tuple whose elements $(s, t) \in \underline{s} \times \underline{t}$ are those pairs with $s \in \underline{s}, t \in \underline{t}$.

Definition 7.6. Given pre-broad posets $S, T$ (cf. Remark 5.2), their tensor product $S \otimes T$ is the pre-broad poset whose underlying set is $S \times T$ and whose relations are generated by relations of the form $\underline{s} \times t \leq(s, t)($ resp. $s \times \underline{t} \leq(s, t))$ for $s \in S, t \in T$ and $\underline{s} \leq s$ (resp. $\underline{t} \leq t)$ a broad relation in $S$ (resp. T).

Proposition 7.7. If $S, T$ are simple broad posets then so is $S \otimes T$. Further, for any non identity broad relation $\left(s_{1}, t_{1}\right) \cdots\left(s_{n}, t_{n}\right) \leq(s, t)$ in $S \otimes T$ one has:

(i) for all $0 \leq i, j \leq n$ it is $s_{i} \leq_{d} s, t_{j} \leq_{d} t$;

(ii) for $i \neq j$ either there exists $\underline{s}$ such that $s_{i} s_{j} \underline{s} \leq s$ or there exists $\underline{t}$ such that $t_{i} t_{j} \underline{t} \leq t$ (or both);

(iii) if both of the pairs $s_{i}, s_{j}$ and $t_{i}, t_{j}$ are $\leq_{d}$-comparable then $i=j$.

Proof. Note first that the "further" conditions suffice to check that $S \otimes T$ is a simple broad poset, i.e. that they imply antisymmetry and simplicity. Indeed, antisymmetry follows from combining (i) with the fact that $\leq_{d}^{S}, \leq_{d}^{T}$ are order relations while simplicity is a particular case of (iii).

(i) follows since the condition holds for generating relations and is preserved by transitivity. Similarly, (ii) holds for generating relations and is readily seen to be preserved by transitivity when applied to relations satisfying (i). Lastly, (iii) follows from (ii) and the $\leq_{d}$-incomparability result in Proposition 5.6 .

Remark 7.8. The main claim in Proposition 7.7 fails for non simple broad posets. As an example, let $S$ be the broad poset $\{a, b\}$ with generating relations $a b \leq a, a b \leq b$ (antisymmetry holds since $\leq$ decreases the size of tuples) and $T$ be $\{c\}$ with generating relation $\epsilon \leq c$. Then $(a, c) \leq(b, c) \leq(a, c)$ hold in $S \otimes T$.

Proposition 7.9. Let $S, T$ be trees. An edge $(s, t) \in S \otimes T$ has one of five types:

(leaf) it is a leaf if both $s \in S, t \in T$ are leaves;

(stump) it is a stump if $s \in S$ is a leaf and $t \in T$ is a stump or vice versa, or if both $s \in S, t \in T$ are stumps;

(leaf node) it is a node if $s \in S$ is a node and $t \in T$ is a leaf or vice versa. In fact $(s, t)^{\uparrow}=s^{\uparrow} \times t$ or $(s, t)^{\uparrow}=s \times t^{\uparrow}$, accordingly;

(null node) it is a node such that $\epsilon \leq(s, t)$ if $s \in S$ is a node and $t \in T$ a stump or vice versa. In fact $(s, t)^{\uparrow}=s^{\uparrow} \times t$ or $(s, t)^{\uparrow}=s \times t^{\uparrow}$, accordingly;

(fork) if $s \in S, t \in T$ are both nodes then there are exactly two maximal $f$ such that $\underline{f}<(s, t)$, namely $s \times t^{\uparrow}$ and $s^{\uparrow} \times t$. We call such $(s, t)$ a fork. 
Proof. Only the fork case requires proof. In fact, it is tautological that only $s \times t^{\uparrow}$ and $s^{\uparrow} \times t$ can possibly be maximal, hence one needs only verify that neither $s \times t^{\uparrow} \leq s^{\uparrow} \times t$ nor $s^{\uparrow} \times t \leq s \times t^{\uparrow}$. This follows since the $S$ coordinates of the pairs in the tuple $s^{\uparrow} \times t$ are $<_{d}$ than those in $s \times t^{\uparrow}$ and vice versa.

In order to simplify notation, we will henceforth write $e^{\uparrow S}=s^{\uparrow} \times t, e^{\uparrow T}=s \times t^{\uparrow}$ and $e^{\uparrow S, T}=s^{\uparrow} \times t^{\uparrow}$.

Proposition 7.10. Let $S, T$ be trees and consider the broad relation

$$
\underline{e}=\left(s_{1}, t_{1}\right)\left(s_{2}, t_{2}\right) \cdots\left(s_{n}, t_{n}\right) \leq(s, t)=e .
$$

in $S \otimes T$. Then:

(i) $\underline{e} \leq e^{\uparrow S}$ (resp. $\underline{e} \leq e^{\uparrow T}$ ) if and only if $s_{i} \neq s, \forall i$ (resp. $\left.t_{i} \neq t, \forall i\right)$;

(ii) $\underline{e} \leq e^{\uparrow S, T}$ if and only if both $s_{i} \neq s$ and $t_{i} \neq t, \forall i$.

Proof. Only the "if" directions need proof, and the proof follows by upward $\leq_{d}$ induction on $s, t$. The base cases of either $s$ or $t$ a leaf are obvious.

Otherwise, let $\underline{e}$ satisfy the "if" condition in (i). Since it must be either $\underline{e} \leq e^{\uparrow S}$ or $\underline{e} \leq e^{\uparrow T}$ we can assume it is the latter case. Writing $t^{\uparrow}=u_{1} \cdots u_{k}$, $\underline{e}=\underline{e}_{1} \cdots \underline{e}_{k}$ so that $\underline{e}_{i} \leq\left(s, u_{i}\right)$ (note that possibly $k=0$ ), the induction hypothesis now yields $\underline{e}_{i} \leq\left(s, u_{i}\right)^{\uparrow S}=s^{\uparrow} \times u_{i}$, and hence

$$
\underline{e}=\underline{e}_{1} \cdots \underline{e}_{k} \leq\left(s^{\uparrow} \times u_{1}\right) \cdots\left(s^{\uparrow} \times u_{k}\right)=s^{\uparrow} \times t^{\uparrow} \leq s^{\uparrow} \times t=e^{\uparrow S} .
$$

The proof of (ii) simply disregards the last inequality in (7.11).

Corollary 7.12. $\underline{e} \leq e^{\uparrow S, T}$ if and only if both $\underline{e} \leq e^{\uparrow S}$ and $\underline{e} \leq e^{\uparrow T}$.

Lemma 7.13. Let $S, T$ be trees. For any $e=(s, t) \in S \otimes T$ there exists a minimum $e^{\lambda} \in(S \times T)^{+}$such that $e^{\lambda} \leq e$. In fact, $e^{\lambda}=s^{\lambda} \times t^{\lambda}$.

Proof. The proof is by $\leq_{d}$ induction on $e$. The case of $s, t$ both leaves is obvious. Otherwise, for any non-identity relation either $f \leq e^{\uparrow S}<e$ or $f \leq e^{\uparrow T}<e$, and the analysis in the proof of Lemma 5.12 applies in either case to show that indeed $s^{\lambda} \times t^{\lambda} \leq \underline{f}$.

\subsection{Subtrees}

Definition 7.14. Let $S, T$ be trees. A subtree of $S \otimes T$ is a tree $U$ together with a broad poset map $U \hookrightarrow S \otimes T$ that is an underlying monomorphism. Further, a subtree is called full if the relations in $U$ coincide with those in its image and in that case we instead write $U \subset S \otimes T$.

We have the following characterization.

Proposition 7.15. $U \stackrel{\varphi}{\rightarrow} S \otimes T$ is full iff for each leaf $l \in U$ it is not $\epsilon \leq \varphi(l)$.

Proof. To simplify notation we will simply write $u$ for both an edge $u \in U$ and its image $\varphi(u) \in S \otimes T$ and instead decorate the broad relations as $\leq^{U}, \leq^{S \otimes T}$, and similarly write $u^{\lambda, U}, u^{\lambda, S \otimes T}$ following Lemmas 5.12 and 7.13 .

We need to show that a broad relation $\underline{u}=u_{1} \cdots u_{n} \leq S \otimes T u$ can not fail the conditions in Lemma 5.12 with respect to $U$. Further, we note that, since $S \otimes T$ 
has a $\leq_{d}$-maximal element $\left(r_{S}, r_{T}\right)$, the proof of Lemma 5.32 applies to show that the two $\leq_{d}$-relations on $\varphi(U)$ coincide. Thus, only condition (iii) of Lemma 5.12 could possibly fail, and this would happen only if $u_{1}^{\lambda, U} \cdots u_{n}^{\lambda, U}$ lacked some of the leaves in $u^{\lambda, U}$. But our hypothesis is that $l^{\lambda, S \otimes T} \neq \epsilon$ for $l$ any leaf of $U$, hence this is impossible.

Definition 7.16. Let $S, T$ be trees. A subtree $U \hookrightarrow S \otimes T$ is called

- elementary if all of its generating broad relations are of the form $e^{\uparrow S} \leq e$ or $e^{\uparrow T} \leq e$

- initial if $U$ contains the "double root" $\left(r_{S}, r_{T}\right) \in S \otimes T$.

Further, a maximal elementary subtree is a subtree that is not contained in any other. Note that, since one can graft new root vertices to $U$, Proposition 5.11 implies that maximal elementary subtrees are necessarily initial.

Remark 7.17. Maximal elementary subtrees are called percolation schemes in [17. Example 9.4].

Remark 7.18. As noted in Proposition 7.9, the relation $\epsilon \leq e$ is a decomposable relation of $S \otimes T$ whenever $e$ is a null node. As a consequence, any elementary tree containing such a generating relation is in fact an inner face of a larger elementary tree.

Lemma 7.19. If $U$ is a full face of $S$, then $U \otimes T \subset S \otimes T$, i.e., $U \otimes T$ contains all broad relations in its image.

Proof. Given a relation $\underline{e}=\left(s_{1}, t_{1}\right) \cdots\left(s_{n}, t_{n}\right) \leq(s, t)=e$ with all $s_{i} \in U$ (but not necessarily $s \in U)$, we first claim that there is a factorization $\underline{e} \leq \underline{u} \times t \leq(s, t)$ with $\underline{u}$ in $U$ and $\underline{u} \leq s$ in $S$. If $s \in U$ one simply takes $\underline{u}=s$. Otherwise Proposition 7.10 yields $\underline{e} \leq e^{\uparrow S} \leq e$ and the claim follows by $\leq_{d}$ induction on $e$.

To check the desired claim that $\underline{e} \leq e$ will be in $U \otimes T$ if $s_{i}, s \in U$, we again argue by $\leq_{d}$ induction on $e$, with the case $\underline{e} \leq e^{\uparrow T} \leq e$ being immediate and the case $\underline{e} \leq e^{\uparrow S} \leq e$ following by the result in the previous paragraph.

Definition 7.20. Let $S, T$ be trees and $A=\left\{a_{i}\right\}, B=\left\{b_{j}\right\}$ subsets of the sets of stumps of $S, T$, respectively, and let $v_{A}=\left\{\epsilon \leq a_{i}\right\}, v_{B}=\left\{\epsilon \leq b_{j}\right\}$ denote the corresponding vertices.

We say that a subtree $U \hookrightarrow S \otimes T$ misses $v_{A}$ and $v_{B}$ if one has a factorization $U \hookrightarrow\left(S-v_{A}\right) \otimes\left(T-v_{B}\right) \hookrightarrow S \otimes T$

Further, if $B=\varnothing$ (resp. $A=\varnothing$ ) we say simply that " $U$ misses $v_{A}$ " (resp. " $U$ misses $\left.v_{B} "\right)$.

Remark 7.21. In [17] similar notions of " $U$ missing an inner edge/leaf vertex are also defined, but we note that (due to Lemma 7.19) those notions are far more straightforward. In fact, as explained in the erratum to the follow up paper [5], the earlier treatment overlooked some subtle properties of stumps.

For instance, note that Corollary 7.24 below implies that the notion " $U$ misses $v_{A}$ and $v_{B}$ " does not coincide with the notion " $U$ misses $v_{A}$ and $U$ misses $v_{B}$ " whenever $A$ and $B$ are both non-empty. 
Lemma 7.22. Let $S, T$ be trees and $A=\left\{a_{i}\right\}, B=\left\{b_{j}\right\}$ subsets of the stumps of $S, T$, respectively. Then a broad relation

$$
\underline{f}=f_{1} f_{2} \cdots f_{k} \leq e
$$

in $S \otimes T$ is a broad relation in $\left(S-v_{A}\right) \otimes\left(T-v_{B}\right)$ if and only if

$$
e^{\lambda,\left(S-v_{A}\right) \otimes\left(T-v_{B}\right)} \leq \underline{f} .
$$

Proof. Only the "if" direction needs proof. We argue by $\leq_{d}$ induction on $e=$ $(s, t)$. The base case, that of $s$ a leaf of $S-v_{A}$ and $T$ a leaf of $T-v_{B}$, is obvious (we note that the proof will follow even when this case is vacuous).

Otherwise, either $f \leq e^{\uparrow S} \leq e$ or $f \leq e^{\uparrow T} \leq e$ and our assumption ensures, respectively, that $s \notin A$ or $t \notin B$. Writing $e^{\uparrow *}$ to denote either $e^{\uparrow S}$ or $e^{\uparrow T}$ as appropriate, this last observation guarantees that the relation $e^{\uparrow *} \leq e$ is in $\left(S-v_{A}\right) \otimes\left(T-v_{B}\right)$. Further, writing $\underline{f}=\underline{f}_{1} \cdots \underline{f}_{k}$ and $e^{\uparrow *}=e_{1} \cdots e_{k}$ so that $f_{i} \leq e_{i}$, the induction hypothesis shows that these last relations are also in $\left(S-v_{A}\right) \otimes\left(T-v_{B}\right)$.

Recalling Proposition 5.30 hence yields the following.

Corollary 7.23. A collection of broad relations of the form $\underline{g}_{i} \leq f_{i}, f_{1} \cdots f_{k} \leq e$ are all in $\left(S-v_{A}\right) \otimes\left(T-v_{B}\right)$ if and only if the composite relation $\underline{g}_{1} \cdots \underline{g}_{k} \leq e$ is.

Corollary 7.24. A subtree $U \hookrightarrow S \otimes T$ misses $v_{A}$ and $v_{B}$ if and only if, for $r$ the root of $U$, one has

$$
r^{\lambda,\left(S-v_{A}\right) \otimes\left(T-v_{B}\right)} \leq r^{\lambda, U} .
$$

Proof. This follows from Corollary 7.23 since any generating relation in $U$ is a factor of $r^{\lambda, U} \leq r$.

The following was first stated in [5. Prop 1.9 in erratum] and proven via a careful combinatorial analysis in 8 . We include here a short broad poset proof. Recall that $\mathrm{d}$ Set $\times \mathrm{d}$ Set $\stackrel{\otimes}{\rightarrow} \mathrm{d}$ Set is defined by setting $\Omega[S] \otimes \Omega[T]=H o m(-, S \otimes T)$ together with the requirement that $\otimes$ commutes with colimits in each variable.

Proposition 7.25. Let $S, T \in \Omega$ be trees which are either (i) both open; (ii) $S=$ $[n]$ is linear. Then the square

$$
\begin{array}{cc}
\partial \Omega[S] \otimes \partial \Omega[T] & \hookrightarrow \Omega[S] \otimes \partial \Omega[T] \\
\mathfrak{\downarrow} & \mathfrak{\downarrow} \\
\partial \Omega[S] \otimes \Omega[T] & \longrightarrow \Omega[S] \otimes \Omega[T]
\end{array}
$$

consists of normal monomorphisms. Further,

$$
\partial \Omega[S] \otimes \Omega[T] \underset{\partial \Omega[S] \otimes \partial \Omega[T]}{\bigsqcup} \Omega[S] \otimes \partial \Omega[T] \leftrightarrow \Omega[S] \otimes \Omega[T]
$$

is also a normal monomorphism.

In particular, (7.26) is a pullback square. 
Proof. Note first that since $\otimes$ commutes with colimits in each variable,

$$
\partial \Omega[S] \otimes \Omega[T]=\operatorname{colim}_{F \in \operatorname{Faces}(S)-\{S\}} \Omega[F] \otimes \Omega[T] .
$$

In the open case, since all faces are full, Lemma 7.19 implies that if $U \subset F_{S} \otimes T$ and $U \subset S \otimes F_{T}$ then it will be $U \subset F_{S} \otimes F_{T}$, showing that (7.27) is a monomorphism whenever (7.26) consists of monomorphisms. By skeletal induction, it thus suffices to check that the right and bottom maps in (7.26) are monomorphisms, and this will follow if for any $U \subset S \otimes T$ there exists a minimal face $F \subset S$ such that $U \subset F \otimes T$. Clearly $F=\left\{s \mid \exists_{t}(s, t) \in U\right\}$ will work once we show that this is indeed a face. This is clear when $U$ is elementary (in which case each vertex of $U$ either adds a vertex to $F$ or nothing at all) and holds in general since any $U$ is an inner face of an elementary subtree.

In the $S=[n]$ linear case the fact that $S$ is open still suffices to conclude that (7.27) being a monomorphism will follow once we show that (7.26) consists of monomorphisms. And, similarly, that $\partial \Omega[n] \otimes \Omega[T] \rightarrow \Omega[n] \otimes \Omega[T]$ is a monomorphism follows by the same argument, building $F$ in the same way. It remains to show that $\Omega[n] \otimes \partial \Omega[T] \rightarrow \Omega[n] \otimes \Omega[T]$ is a monomorphism. We note that the projection $\pi:[n] \otimes T \rightarrow T$ given by $\pi(k, e)=e$ is a map of broad posets. Given $U \hookrightarrow[n] \otimes T$ we claim that $\pi(U)$ is the minimal face such that $U \hookrightarrow[n] \otimes \pi(U)$, noting that this is implied by the more general claim that $U \hookrightarrow[n] \otimes F$ iff $\pi(U) \rightarrow F$. It now suffices to check this when $F$ is a maximal face, with the case of $F$ full being obvious from Lemma 7.19 and the stump outer face case following from Corollary 7.24 .

The claim that (7.26) is a pullback square is elementary (compare with the proof of [5, Prop. 1.9]).

\subsection{Pushout product filtrations}

This section features our main technical proofs, namely the proof of Theorem 7.1 and the related but simpler proof of Theorem 7.2 .

The majority of the ideas in this section are adapted from the (rather long) proof of [17, Prop. 9.2], but here we will need to significantly repackage those ideas. To explain why, we note that the filtrations in the proof of [17, Prop. 9.2] are actually divided into three nested tiers: an outermost tier described immediately following [17, Cor. 9.3], an intermediate tier described in the proof of [17, Lemma 9.9] and an innermost tier described in the proof of [17, Lemma 9.7]. However, in the equivariant case $G$ acts transversely to these tiers, i.e. one can not attach dendrices at an inner tier stage without also attaching dendrices in a different outer tier stage.

Our solution will be to encode the top two filtration tiers as a poset $\mathrm{IE}_{G \xi}(S \otimes$ $T$ ) on which $G$ acts (to handle the lower tier). To improve readability, however, we first describe our repackaged proof in the non-equivariant case, then indicate the (by then minor) necessary equivariant modifications.

We will make use of an order relation on elementary subtrees (cf. Definition [7.16) of $S \otimes T$.

Definition 7.29. Write $V \leq_{\text {lex }} U$ whenever $U$ is obtained from $V$ by replacing the intermediate edges in a string of broad relations $e^{\uparrow S, T} \leq e^{\uparrow S} \leq e$ occurring in $V$ with the intermediate edges in $e^{\uparrow S, T} \leq e^{\uparrow T} \leq e$ occurring in $U$. An illustrative 
diagram follows.
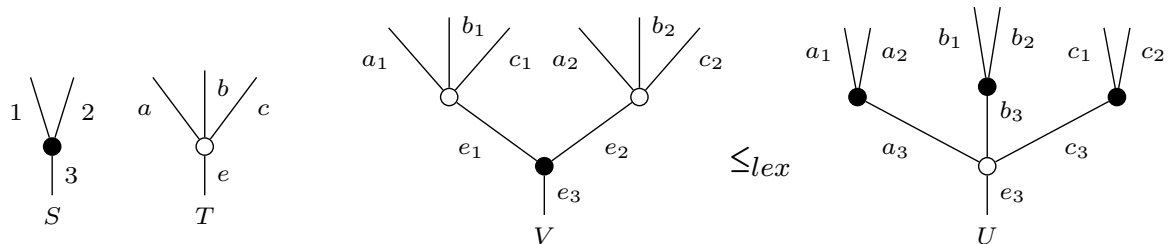

Remark 7.30. The $\leq_{l e x}$ relation is compatible with the grafting procedure in Remark 5.16. In particular, we will throughout assume that a relation $V \leq_{l e x} U$ can be built by first ungrafting $U$ (Proposition 5.19), then applying $\leq_{l e x}$ relations to each piece, and finally regrafting the pieces to obtain $V$.

In what follows we refer to generating relations of the form $e^{\uparrow S} \leq e$ (resp. $\left.e^{\uparrow T} \leq e\right)$ in an elementary subtree as $S$-vertices (resp. $T$-vertices). Also, given vertices $v=\left(e^{\uparrow *} \leq e\right), w=\left(f^{\uparrow *} \leq f\right)$ we write $v \leq_{d} w$ if $e \leq_{d} f$.

Proposition 7.31. Suppose $T$ is open (i.e. has no stumps). Then $\leq_{l e x}$ induces a partial order on the set of elementary subtrees of $S \otimes T$.

Further, $\leq_{\text {lex }}$ together with the inclusion $\rightarrow$ assemble into a partial order as well, and we denote this latter order simply by $\leq$.

Proof. One needs only check antisymmetry. Let $g(U)$ count pairs $\left(v_{S}, v_{T}\right)$ of a $S$-vertex and $T$-vertex in $U$ such that $v_{S} \leq_{d} v_{T}$. Since the generating relations of $\leq_{l e x}$ strictly increase $g, \leq_{l e x}$ is a partial order. Similarly, letting $h(U)=\#\{$ stumps of $U\}+\sum_{l \in\{\text { leaves and stumps of } U\}} d(l, r)$ (where $d(-, r)$ denotes "distance to the root", measured in generating $\leq_{d}$ relations), $\hookrightarrow$ increases $h$ and $\leq_{l e x}$ either (i) preserves $h$ if $e$ (as in Definition 7.29) is a fork; (ii) is an instance of $\hookrightarrow$ if $e$ is a null node (since $T$ is assumed open). Thus $\leq$ is a partial order.

Remark 7.32. Note that if $T$ is not open, then by Remark 7.18 it is possible for the combination of $\leq_{l e x}$ and $\hookrightarrow$ to fail antisymmetry.

Henceforth we will let $\xi$ denote a fixed inner edge of $T$.

Definition 7.33. An initial elementary subtree $U \hookrightarrow S \otimes T$ (cf. Definition 7.16) is called $\xi$-internal if it contains an edge of the form $(s, \xi)$, abbreviated as $\xi_{s}$, and the $T$-vertex $\xi_{s}^{\uparrow T} \leq \xi_{s}$.

For $T$ open, we will denote the subposet of such trees by $\left(\operatorname{IE}_{\xi}(S \otimes T), \leq\right)$.

Further, when $S$ is open one can modify the order in $\operatorname{IE}_{\xi}(S \otimes T)$ by reversing the $s_{\text {lex }}$ order (but not the $\hookrightarrow$ order). The resulting poset will be denoted $\mathrm{IE}_{\xi}^{\text {oplex }}(S \otimes T)$.

Lemma 7.34. Suppose $T$ is open. Let $U \hookrightarrow S \otimes T$ be an elementary subtree with root vertex a T-vertex $e^{\uparrow T} \leq e$ and suppose that $e^{\lambda, U} \leq e^{\uparrow S}$ (or, by Proposition 7.10, that none of the leaves in $e^{\lambda, U}$ have the same $S$ coordinate as e).

Then there exists an elementary subtree $V$ such that $V \leq_{l e x} U$ and $V$ contains the relations $e^{\uparrow S, T} \leq e^{\uparrow S} \leq e$.

Proof. We argue by induction on the sum of the distances (cf. proof of Proposition 7.31) between the leaves and stumps of $U$ and its root $e$. The base case, that of $U$ the elementary tree generated by $e^{\uparrow S, T} \leq e^{\uparrow T} \leq e$, is obvious. 
Otherwise, writing $e=(s, t)$, for each $t_{i} \in t^{\uparrow}$ either $\left(s, t_{i}\right)^{\uparrow S} \leq^{U}\left(s, t_{i}\right)$ or $\left(s, t_{i}\right)^{\uparrow T} \leq^{U}\left(s, t_{i}\right)$. Applying the induction hypothesis to each of the subtrees $U^{\leq\left(s, t_{i}\right)}$ (cf. Proposition 5.19) in the latter case yields trees $W_{i} \leq_{l e x} U^{\leq\left(s, t_{i}\right)}$, which after grafted yield a tree $W \leq_{l e x} U$ such that $W$ contains all relations $\left(s, t_{i}\right)^{\uparrow S} \leq\left(s, t_{i}\right)$. But now $W$ contains the relations $e^{\uparrow S, T} \leq e^{\uparrow T} \leq e$ and hence a final generating $\leq_{\text {lex }}$ relation yields the desired $V \leq_{\text {lex }} W \leq_{\text {lex }} U$.

Example 7.35. A typical illustration of the previous result follows.
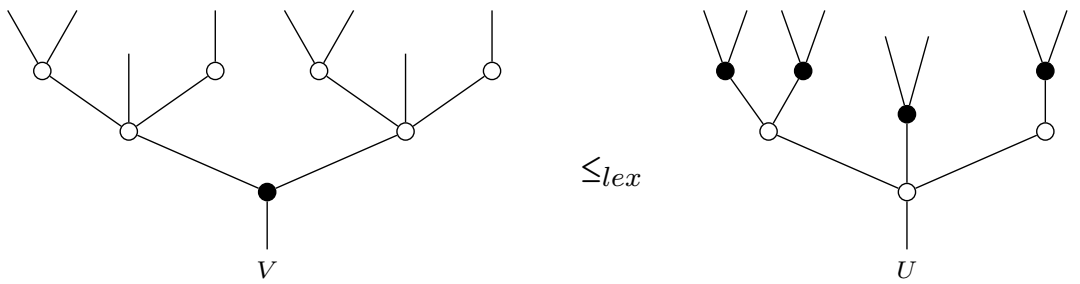

Lemma 7.36. Suppose $U \hookrightarrow S \otimes T, V \hookrightarrow S \otimes T$ are subtrees with common leaves and root. Then $F=U \cap V$ defines a full face of both $U$ and $V$.

Proof. As a set, $F$ could alternatively be defined as the underlying set of the composite inner face of $U$ that removes all inner edges of $U$ not in $V$, or vice versa. Thus, the real claim is that both constructions yield the same broad relations. Noting that the $\leq_{d}$ order relations on $U, V$ are induced from $S \otimes T$ (as argued in the proof of Proposition 7.15), this follows from Lemma 5.12,

Lemma 7.37. Suppose $T$ is open. If $F$ is a common face (resp. inner face) of two elementary subtrees $U, V$, then $F$ is also a face (resp. inner face) of an elementary subtree $W$ such that $W \leq U, W \leq V$ (resp. $\left.W \leq_{\text {lex }} U, W \leq_{\text {lex }} V\right)$. In fact, in the inner face case the $\leq_{l e x}$ inequalities factor through generating $\leq_{l e x}$ inequalities involving only trees having $F$ as an inner face.

Proof. Letting $r, \underline{l}$ denote the root and leaves of $F$, by Corollary 5.23 one can replace $U, V$ with $U_{\nless \underline{l}}^{\leq r}, V_{\nless \underline{l}}^{\leq r}$, reducing to the case where $F, U, V$ have exactly the same leaves and root. Thus, by Lemma 7.36 we are free to assume $F=U \cap V$.

If the root vertices $r^{\uparrow U} \leq r, r^{\uparrow V} \leq r$ coincide, the result follows by induction on $\leq$. Otherwise, we can assume that the root vertex of $U$ is $r^{\uparrow S} \leq r$ and that of $V$ is $r^{\uparrow T} \leq r$. Lemma 7.34 now applies to $V_{\nless r^{\uparrow F}}$, and one can hence build $W \leq_{\text {lex }} V$ with a strictly larger intersection with $U$, finishing the proof. $\bar{b} \in B$.

Recall that a subset $B$ of a poset $\mathcal{P}$ is called convex if $\bar{b} \leq b$ and $b \in B$ implies

Proposition 7.38. Let $S, T$ be trees and $\xi \in T$ an inner edge. Further, assume that either both $S$ and $T$ are open or that one of them is linear. Set

$$
A=\Omega[S] \otimes \Lambda^{\xi}[T] \coprod_{\partial \Omega[S] \otimes \Lambda^{\xi}[T]} \partial \Omega[S] \otimes \Omega[T]
$$

and regard $A$ and the $\Omega[V]$ below as subpresheaves of $\Omega[S] \otimes \Omega[T]$.

Then, for any convex subsets $B \subset B^{\prime}$ of the poset $\operatorname{IE}_{\xi}(S \otimes T)$ (or, in the special case of $S$ a linear tree and $T$ not open, of the poset $\operatorname{IE}_{\xi}^{\text {oplex }}(S \otimes T)$ ), one has that

$$
A \cup \bigcup_{V \in B} \Omega[V] \rightarrow A \cup \bigcup_{V \in B^{\prime}} \Omega[V]
$$


is an inner anodyne extension.

To simplify notation we will throughout the proof suppress $\Omega$ from the notation, e.g., $A \cup \cup_{V \in B} \Omega[V]$ will be denoted simply as $A \cup \cup_{V \in B} V$.

The key to the proof is given by the following couple of lemmas.

Lemma 7.39. Suppose that either $S, T$ are both open or that $T$ is linear.

Then, for $U \in \mathrm{IE}_{\xi}(S \otimes T)$ and $B$ convex such that $\{V \mid V<U\} \subset B$, any edge $\xi_{s}$ of $U$ with vertex $\xi_{s}^{\uparrow T} \leq \xi_{s}$ is a characteristic edge (in the sense of 17 , Lemma 9.7]), i.e., for each inner face $F$ of $U$ containing the edge $\xi_{s}$, then $F$ is in $A \cup \cup_{V \in B} V$ if and only if $F-\xi_{s}$ is.

Proof. Suppose first that $F-\xi_{s}$ is in $A$ but that $F$ is not.

Either $F-\xi_{s} \hookrightarrow S^{\prime} \otimes T$ or $F-\xi_{s} \hookrightarrow S \otimes T^{\prime}$ for $S^{\prime} \hookrightarrow S, T^{\prime} \hookrightarrow T$ some maximal subface where in the latter case $T^{\prime} \neq T-\xi$. Considering the cases in Notation 5.41, the stump cases are excluded by Corollary 7.24 and in the full cases Lemma 7.19 implies that the only possibility is for $F-\xi_{s}$ to have no edge with $S$-coordinate $s$ while $F$ does. Further, if $s$ were to be a root or leaf of $S$, $F-\xi_{s}$ would still contain a root or leaf with $S$-coordinate $s$ (this latter case uses the fact that $T$ is open). Thus, the only possibility is $F-\xi_{s} \hookrightarrow(S-s) \otimes T$ for $s$ an inner edge of $S$.

Now let $\xi_{s}^{\uparrow<s}=e_{1} \cdots e_{k}$ consist of the $\leq_{d}$-maximal $e_{i}=\left(s_{i}, t_{i}\right)$ such that both $e_{i}<_{d} \xi_{s}$ and $s_{i}<_{d} s$ and consider the subtree $U_{\nless \xi_{s} \uparrow s}^{\leq \xi_{s}}$. Then: (i) this tree has no leaf with $S$ coordinate $s$, or else that would be a leaf of $U$ (Remark 5.22), and thus also of $F$, so that it could not be $F-\xi_{s} \rightarrow(S-s) \otimes T$; (ii) the leaf tuple of this tree is hence $\xi_{s}^{\uparrow<s}$; (iii) by definition of $\xi_{s}^{\uparrow<s}$, all inner edges of this tree have $S$ coordinate $s$. But the condition $F-\xi_{s} \rightarrow(S-s) \otimes T$ now implies that $F$ contains none of the inner edges of $U_{\left\langle\xi_{s}^{<s}\right.}^{\leq \xi_{s}}$, so that Lemma 7.34 implies that $F$ is a subface of some $V<_{l e x} U$, hence contained in $A \cup \cup_{V \in B} V$.

Suppose now that $F-\xi_{s}$ is a subface of some $V \in B$. By Lemma 7.37 (and its proof) we can assume that in fact $F-\xi_{s}$ is an inner subface of $V$ and $V<_{\text {lex }} U$. Further, by the "in fact" part of Lemma 7.37 one can also assume that this is a generating $\leq_{l e x}$ relation. But then $V$ necessarily contains $\xi_{s}$, since generating $\leq_{l e x}$ relations do not add edges whose vertex is a $T$-vertex. Thus $F-\xi_{s} \hookrightarrow V$ implies $F \hookrightarrow V$.

Example 7.40. The following is a typical tree illustration of $U_{\Varangle \xi_{s}^{\uparrow<s}}^{\leq \xi_{s}}$. In words, this subtree always: (i) has $S$-vertices (in black), all of the same arity, below its leaves; (ii) has a $T$-vertex above its root $\xi_{s}$ (in white); (iii) all its remaining vertices are $T$-vertices (so that the edges marked $s$ have $S$-coordinate $s$ ).

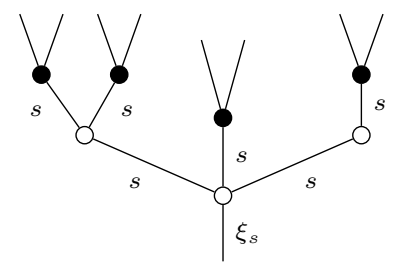

Remark 7.41. For the previous proof to work it is crucial for the tree $U_{\substack{<\xi_{s} \\\langle<}}^{<\xi_{s}}$ to in fact have inner edges, as is ensured by the fact that $\xi$ is not a stump of $T$. In this latter case we will instead need to use the following alternative lemma. 
Lemma 7.42. Suppose that $S$ is a linear tree (i.e. $S \simeq[n]$ for $[n] \in \Delta$ ).

Then for $U \in \mathrm{IE}_{\xi}^{\text {oplex }}(S \otimes T)$ the $\leq_{d}$-maximal edge of $U$ of the form $\xi_{s}$ is a characteristic edge (in the sense of Lemma 7.39).

Proof. Since $S$ is linear we will for simplicity label its edges as $0 \leq 1 \leq \cdots \leq n$.

Suppose first that $F-\xi_{s}$ is in $A$, so that repeating the argument in the proof of Lemma 7.39 we conclude it must be $F-\xi_{s} \rightarrow(S-s) \otimes T$ for $s<n$ (note that this makes sense even if $s=0$ is the leaf of $S$, which must be considered if $T$ is not open).

Since $s \neq n$, one can choose a $\leq_{d}$-minimal edge $a_{s+1}$ of $U$ such that $\xi_{s} \leq_{d} a_{s+1}$. Then: (i) the characterization of $\xi_{s}$ implies $a \neq \xi$; (ii) the characterization of $a_{s+1}$ implies that $U$ contains the $S$-vertex $a_{s}=a_{s+1}^{\uparrow S} \leq a_{s+1}$; (iii) since $U$ contains both $\xi_{s}$ and $a_{s}$ it contains the $T$-vertex $a_{s}^{\uparrow T} \leq a_{s}$, which can be rewritten as $a_{s+1}^{\uparrow S, T} \leq a_{s+1}^{S} . U$ therefore contains the relations $a_{s+1}^{\uparrow S, T} \leq a_{s+1}^{\uparrow S} \leq a_{s+1}$, which since $F$ must collapse $a_{s}=a_{s+1}^{\uparrow S}$ yields that $F$ is a subface of the tree $V \leq_{\text {oplex }} U$ obtained by replacing $a_{s}=a_{s+1}^{\uparrow S}$ with $a_{s+1}^{\uparrow T}$.

The case of $F-\xi_{s}$ a subface of some $V \in B$ follows by an argument identical to that in the proof of Lemma 7.39, except now noting that generating $\leq_{\text {oplex }}$ relations do not add edges whose vertex is a $S$-vertex.

Proof of Proposition 7.38. Without loss of generality we can assume that $B^{\prime}$ is obtained from $B$ by adding a single $\xi$-internal initial elementary tree $U$ with $\xi_{s}$ its corresponding edge.

We first note that the outer faces of $U$ are in $A \cup \bigcup_{V \in B} V$. Since a maximal outer face $\bar{U} \leftrightarrow U$ is always still elementary, $\bar{U}$ will be $\xi$-internal initial elementary unless (i) $\bar{U}=U^{\leq\left(e, r_{T}\right)}$ (resp. $\bar{U}=U^{\leq\left(r_{S}, e\right)}$ ) is a root vertex face, in which case $\bar{U} \hookrightarrow\left(S^{\leq e}\right) \otimes T$ (resp. $\left.\bar{U} \hookrightarrow S \otimes\left(T^{\leq e}\right)\right)$ and is hence in $A$; (ii) $\bar{U}=U_{\nless \xi_{s}}$ and $\bar{U}$ is no longer $\xi$-internal since it contains no $T$-vertices of the form $\xi_{\tilde{s}}^{\uparrow T} \leq \xi_{\tilde{s}}$. But then it would be $\bar{U} \rightarrow S \otimes T_{\nless \xi}$ (by either Lemma 7.19 or Corollary 7.24) and thus $\bar{U}$ is in $A$.

Finally, we let $\operatorname{lnn}_{\hat{\xi}_{s}}(U)$ denote the poset of inner faces of $U$ removing only edges other than $\xi_{s}$. We claim that for any convex subsets $C \subset C^{\prime} \subset \operatorname{lnn}_{\hat{\xi}_{s}}(U)$ the map

$$
A \cup \bigcup_{V \in B} V \cup \bigcup_{W \in C} W \rightarrow A \cup \bigcup_{V \in B} V \cup \bigcup_{W \in C^{\prime}} W
$$

is inner anodyne. We argue by induction on $C$ and again we can assume that $C^{\prime}$ is obtained from $C$ by adding a single $X \in \operatorname{lnn}_{\hat{\xi}_{s}}(U)$ not yet in the domain of (7.43). The concavity of $C, C^{\prime}$ and the characteristic edge condition in Lemmas 7.39, 7.42 then imply that the only faces of $X$ not in the source of (7.43) are precisely $X$ and $X-\xi_{s}$, showing that (77.43) is a pushout of $\Lambda^{\xi_{s}}[X] \rightarrow \Omega[X]$, finishing the proof.

In the $G$-equivariant case, given an inner edge orbit $G \xi$, we write $\mathrm{IE}_{G \xi}(S \otimes T)$ for the poset of initial elementary trees containing at least one $T$-vertex of the form $(g \xi)_{s}^{\uparrow T} \leq(g \xi)_{s}$ (alternatively, one has $\mathrm{IE}_{G \xi}(S \otimes T)=\cup_{g \in G} \mathrm{IE}_{g \xi}(S \otimes T)$ ). Note that in this case the group $G$ acts on the poset $\operatorname{IE}_{G \xi}(S \otimes T)$ as well. The following is the equivariant version of Proposition 7.38

Proposition 7.44. Let $S, T \in \Omega_{G}$ be $G$-trees and $\xi \in T$ an inner edge. Further, assume that either both $S$ and $T$ are open or that one of them is linear (i.e. of 
the form $G / H \cdot[n])$. Set

$$
A=\Omega[S] \otimes \Lambda^{G \xi}[T] \coprod_{\partial \Omega[S] \otimes \Lambda^{G \xi}[T]} \partial \Omega[S] \otimes \Omega[T] .
$$

and regard $A$ and the $\Omega[V]$ below as subpresheaves of $\Omega[S] \otimes \Omega[T]$.

Then for any $G$-equivariant convex subsets $B \subset B^{\prime}$ of $\operatorname{IE}_{G \xi}(S \otimes T)$ (or, in the special case of $S$ a linear tree and $T$ not open, of $\left.\mathrm{I}_{G \xi}^{\text {oplex }}(S \otimes T)\right)$ one has that

$$
A \cup \bigcup_{V \in B} \Omega[V] \rightarrow A \cup \bigcup_{V \in B^{\prime}} \Omega[V]
$$

is a $G$-inner anodyne extension.

Again we will suppress $\Omega$ from the notation of the proof.

Proof. Note that we are free to assume $S, T \in \Omega^{G} \subset \Omega_{G}$, i.e. that $S, T$ are actual trees with a $G$-action rather than $G$-indecomposable forests. Indeed, otherwise writing $S \simeq G \cdot{ }_{H} S_{e}, T \simeq G \cdot{ }_{K} T_{e}$ for $S_{e} \in \Omega^{H}, T_{e} \in \Omega^{K}$ yields a decomposition

$$
S \otimes T \simeq \coprod_{[g] \in H \backslash G / K} G \cdot H \cap g K g^{-1} S_{e} \otimes g T_{e},
$$

where when regarding $S_{e}, g T_{e} \in \Omega^{H \cap g K g^{-1}}$ we omit the forgetful functors.

In analogy with the non-equivariant case we can assume $B^{\prime}$ is obtained from $B$ by adding the $G$-orbit of a single $\xi$-internal initial elementary tree $U$ with $\xi_{s}$ the corresponding edge. Let $H \leq G$ denote the $G$-isotropy of $U$ in $\operatorname{IE}_{G \xi}(S \otimes T)$.

That the outer faces of any of the conjugates $g U$ are in $A \cup \cup_{V \in B} V$ follows by the corresponding non-equivariant argument in the proof of Proposition 7.38 ,

The key is now to prove the equivariant analogues of Lemmata 7.39 and 7.42. stating that $H \xi_{s}$ is a characteristic edge orbit of $U$, i.e., that for each inner face $F$ of $U$ with isotropy $\bar{H} \leq H$ and containing an edge $(h \xi)_{(h s)} \in H \xi_{s}$, then $F$ is in $A \cup \bigcup_{V \in B} V$ iff $F-\bar{H}(h \xi)_{(h s)}$ is (note the condition on isotropy). By equivariance, we may without loss of generality assume that $F$ contains $\xi_{s}$ itself.

When proving the equivariant analogue of Lemma 7.39 , in the case of $F-\bar{H} \xi_{s}$ in $A$ the argument in that proof yields that $F$ itself must already lack all the

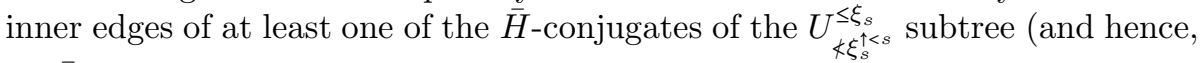
by $\bar{H}$-equivariance, all of them) and therefore applying Lemma 7.34 again shows that $F$ is a subface of some $V<_{\text {lex }} U$. In the case $F-\bar{H} \xi_{s}$ in some $V<$ $U$, repeating the argument in the proof we can again assume $V<_{l e x} U$ via a generating $\leq_{l e x}$ relation and such $V$ must likewise contain all the edges in $H \xi_{s}$.

Proving the equivariant analogue of Lemma 7.42 requires no changes to the proof, since defining $a_{s+1}$ in the same way one still concludes that $F$ must lack $a_{s}$ (in fact, $F$ must lack $\bar{H} a_{s}$, though that fact in not needed).

Lastly, we equivariantly modify the last two paragraphs in the proof of Proposition 7.38, setting $\ln n_{\overline{H \xi_{s}}}(U)$ to be the $H$-poset of inner faces of $U$ lacking only edges not in $H \xi_{s}$, we show by induction on $H$-equivariant concave subsets $C \subset C^{\prime} \subset \operatorname{lnn}_{\overline{H \xi_{s}}}(U)$ that the map

$$
A \cup \bigcup_{V \in B} V \cup\left(\bigcup_{g \in G, W \in C} g W\right) \rightarrow A \cup \bigcup_{V \in B} V \cup\left(\bigcup_{g \in G, W \in C^{\prime}} g W\right)
$$


is inner $G$-anodyne. Again we can assume that $C^{\prime}$ is obtained from $C$ by adding the $H$-orbit of a single $X$ with $H$-isotropy $\bar{H} \leq H$ and not in the domain of (7.45) (further, $X$ can be chosen to contain $\xi_{s}$ ). At this point an extra equivariant argument is needed: one needs to know that the $G$-isotropy of $X$ coincides with its $H$-isotropy $\bar{H}$. To see this, note that if it was otherwise then $X$ would be contained in both $U$ and a distinct conjugate $g U$, and Lemma 7.37 would imply that $X$ is already in the domain of (7.45). Finally, repeating the "characteristic edge (orbit)" argument we see that the map (7.45) is a pushout of

$$
G \cdot \bar{H}\left(\Lambda^{\bar{H} \xi_{s}}[X] \rightarrow \Omega[X]\right),
$$

finishing the proof.

We now adapt the previous proof to deduce the easier Theorem 7.2

Proof of Theorem 7.2. The argument follows by attempting to follow the proof of Proposition 7.44 when $T=[1]$ and $\xi=1$ (note that Definition 7.33 still makes sense, although the edge $1_{s}$ of $U$ may now possibly be the root, hence not internal). The only case where (the equivariant analogue) of Lemma 7.39 does not provide a characteristic edge orbit is when the root vertex of $U$ is $1_{r}^{\uparrow T} \leq 1_{r}$, in which case $U$ will be in $A$ unless (using the notation in the proof of Proposition (7.25) it is $\pi(U)=S$, so that in fact it is $U=S \otimes\{0\} \cup\left\{1_{r}\right\}$. Denoting this latest $U$ as $S \star \eta$ and noting that it is the maximum of the poset $\mathrm{IE}_{\xi}(S \otimes[1])$ one concludes that letting $A \rightarrow B$ denote

$$
A \rightarrow A \cup \bigcup_{V \in \mathrm{I}_{\xi}(S \otimes[1]), V \neq S \star \eta} V
$$

this is indeed inner $G$-anodyne. The pushout (7.3) follows by noting that the only face of $S \star \eta$ not in $B$ is $(S \star \eta)-\eta$ (in fact, the only other face $F$ such that $\pi(F)=S$ is $(S \star \eta)-0_{r}$, which is a common face of the elementary tree $V$ obtained by applying a $\leq_{l e x}$ relation to the root of $\left.S \star \eta\right)$.

\subsection{Dendroidal join}

We now turn to the equivariant version of the dendroidal join $\star$ discussed in [5, §4], which will be needed to understand the last piece of the filtration in Theorem 7.2, We recall that several categories of forests were discussed in $\$ 5.2$.

Definition 7.46. Given an object $F \in \Phi$ and $[n] \in \Delta$ we define $F \star[n] \in \Omega$ as the broad poset having underlying set $F \amalg[n]$ and relations

- $e_{1} \cdots e_{n} \leq e$ if $e_{i}, e \in F$ and $e_{1} \cdots e_{n} \leq^{F} e$;

- $i \leq j$ if $i, j \in[n]$ and $i \leq^{[n]} j$;

- $e_{1} \cdots e_{n} \leq i$ if $e_{j} \in F, i \in[n]$ and $e_{1} \cdots e_{n} \leq^{F} \underline{r}_{F}$. 
Example 7.47. As explained in [5, §4.3], one can readily visualize $\star$ when using tree diagrams, such as in the following example.
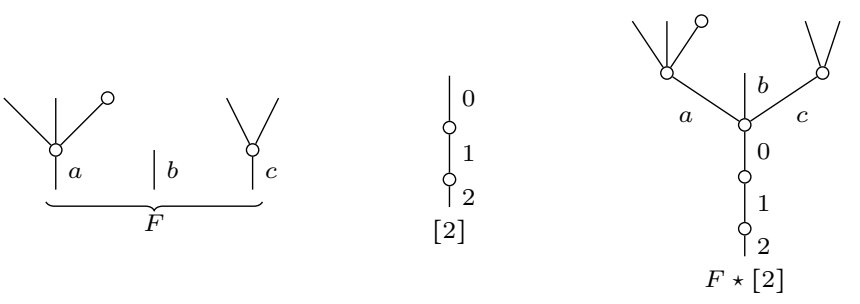

Further, note that when $F=\varnothing$ is the empty forest, it is $\underline{r}_{F}=\epsilon$, and since $\epsilon \leq \epsilon$, $\varnothing \star[n]$ adds a stump at the top of $[n]$.

$$
\begin{gathered}
0 \\
0 \\
1 \\
9 \\
\varnothing *[2]
\end{gathered}
$$

We now discuss the functoriality of $\star$. As implicit in the discussion in 5 , $\S 4.5], \star$ is only functorial with respect to some maps of forests. Indeed, it is clear from the third condition in Definition 7.46 that $\star$ will be functorial in $F$ precisely with respect to the maps in $\Phi_{w}$.

Moreover, the canonical inclusions $[n] \rightarrow F \star[n], F \rightarrow F \star[n]$ can be encoded thusly: letting $\Delta_{+}, \Phi_{w+}, \Phi_{i+}$ denote the categories $\Delta, \Phi_{w}, \Phi_{i}$ together with an additional initial object + , one has the following.

Proposition 7.48. * defines a bifunctor

$$
\Phi_{w+} \times \Delta_{+} \stackrel{-\star-}{\longrightarrow} \Phi_{i+}
$$

such that $+\star[n]=[n], F \star+=F$.

Note that $\star$ usually lands in $\Omega_{+}$, the only exceptions occurring when the second input is the additional initial object.

We now extend the join operation to presheaves by defining

$$
\mathrm{fSet}_{w} \times \mathrm{sSet} \stackrel{-\star-}{\longrightarrow} \mathrm{dSet}
$$

to be the composite (writing $u: \Xi \hookrightarrow \Xi_{+}$for the inclusion and $u^{*}$ :Set $\Xi_{+}^{\text {op }} \rightleftarrows$ Set ${ }^{\Xi^{o p}}: u_{*}$ for the standard adjunction)

$$
\mathrm{fSet}_{w} \times \operatorname{SSet} \stackrel{u_{*} \times u_{*}}{\longrightarrow} \operatorname{Set}^{\Phi_{w+}^{o p}} \times \operatorname{Set}^{\Delta_{+}^{o p}} \stackrel{\times}{\rightarrow} \operatorname{Set}^{\Phi_{w+}^{o p} \times \Delta_{+}^{o p}} \stackrel{\operatorname{Lan}_{\star}}{\longrightarrow} \operatorname{Set}^{\Phi_{i+}^{o p}} \stackrel{u^{*}}{\longrightarrow} \mathrm{dSet} .
$$

Remark 7.50. Unpacking (7.49) one can write (cf. [13, Defn. 1.2.8.1])

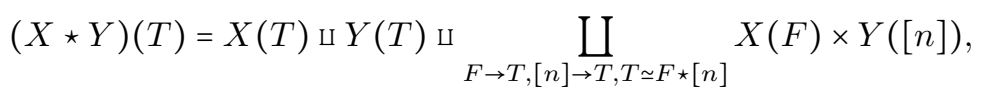

where $Y(T)=\varnothing$ when $T$ is not linear. 
Remark 7.52. Due to the passage through the $(-)_{+}$categories in (7.49), $\mathrm{fSet}_{w} \times$ sSet $\stackrel{-\star-}{\longrightarrow}$ dSet does not preserve colimits in each variable. Rather, the functors $F \star(-),(-) \star[n]$ preserve colimits when mapping into the under categories $\mathrm{dSet}_{F /}, \mathrm{dSet}_{[n] /}$. Therefore, $\star$ does nonetheless preserve connected colimits in each variable.

The following is an equivariant generalization of the key technical lemma [5, Lemma 4.10] combined with key arguments in the proof of [5, Thm. 4.2].

Proposition 7.53. Let $A \stackrel{f}{\rightarrow} B$ be a normal monomorphism in $\mathrm{fSet}_{w}^{G}$ (defined as in Proposition $6.5($ iii) ) and $C \stackrel{g}{\rightarrow} D$ be a left anodyne map in sSet. Then

$$
f \square^{\star} g: A \star D \coprod_{A \star C} B \star C \rightarrow C \star D
$$

is $G$-inner anodyne.

Proof. That $f \square^{\star} g$ is indeed a monomorphism whenever $f, g$ are monomorphisms follows directly from (7.51).

In lieu of Remark 7.52 concerning connected colimits, it suffices to consider the case where $f, g$ have the form $\partial \Phi_{w}[F] \rightarrow \Phi_{w}[F]$ and $\Lambda^{i}[n] \rightarrow \Delta[n], 0 \leq i<n$. But in that case $f \square^{\star} g$ is simply the inner horn inclusion

$$
\Lambda^{i}(F \star[n]) \rightarrow \Omega(F \star[n]) .
$$

Proof of Theorem 7.4. First note that the conditions on $S$ are equivalent to saying that $S \simeq F \star[1]$ for some $F \in \Phi_{w}^{G}$. One can thus rewrite the left vertical map in Theorem 7.4 as

$$
\left(\Lambda^{1}[1] \rightarrow \Delta[1]\right) \square^{\star}\left(\varnothing \rightarrow \Phi_{w}[F]\right)
$$

and denoting by $A \backslash(-)$ the right adjoint to sSet $\stackrel{A \star(-)}{\longrightarrow} \mathrm{dSet}_{A \star+/}^{G}$ standard adjunction arguments allows us to convert (7.5) into the equivalent lifting problem
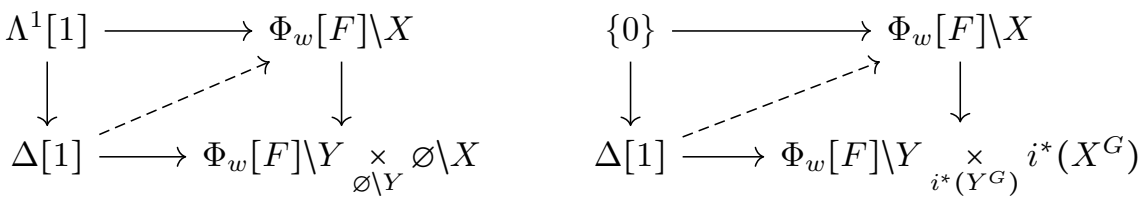

where the right hand diagram merely simplifies the notation on the left: $\varnothing \backslash Z \simeq$ $i^{*}\left(Z^{G}\right)$, where we caution that $\varnothing \neq \Phi_{w}[\varnothing]$, the former being the empty presheaf and the latter the representable presheaf on the empty forest.

Standard repeated applications of Proposition 7.53 (setting $A=\varnothing$ or $Y=$ * as needed) yield that: (i) $\Phi_{w}[F] \backslash X \rightarrow i^{*}\left(X^{G}\right), \Phi_{w}[F] \backslash Y \rightarrow i^{*}\left(Y^{G}\right)$ are left fibrations; (ii) $\Phi_{w}[F] \backslash X, \Phi_{w}[F] \backslash Y$ are left fibrant and thus $\infty$-categories; (iii) the rightmost map $\Phi_{w}[F] \backslash X \rightarrow \Phi_{w}[F] \backslash Y \times_{i^{*}\left(Y^{G}\right)} i^{*}\left(X^{G}\right)$ in (7.54) is a left fibration. Therefore, the map $\Phi_{w}[F] \backslash Y \times_{i^{*}\left(Y^{G}\right)} i^{*}\left(X^{G}\right) \rightarrow i^{*}\left(X^{G}\right)$ is itself a left fibration (it is a pullback of $\Phi_{w}[F] \backslash Y \rightarrow i^{\star}\left(Y^{G}\right)$ ) and since left fibrations conserve equivalences [12, Prop. 2.7] the image of the lower map in (7.54) is a equivalence. The result now follows since equivalences can be lifted over left fibrations between $\infty$-categories [12, Props. 2.4 and 2.7]. 
Remark 7.55. The interested reader may note that Proposition 7.53 and Theorem 7.4 have notably shorter proofs than the analogue results [5, Lemma 4.10] and [5. Thm. 4.2]. In fact, some of our arguments closely resemble the proof for the simplicial case as found in [13, Prop. 1.2.4.3]. The brevity of these arguments is the reason for our introduction of the forest category $\Phi_{w}$ : from our perspective, many of the arguments in [5] are replaced with the task of showing that the boundaries $\partial \Phi_{w}[F]$ satisfy the usual formal properties.

\section{Model structure on equivariant dendroidal sets}

We now adapt the treatment in [5] to equip the category $\mathrm{dSet}^{G}$ with a model structure where the cofibrations are the normal monomorphisms (cf. Prop. 6.5) and the fibrant objects are the $G$ - $\infty$-operads (cf. Defn. 6.12). Since we have already established the equivariant analogues of the main technical results needed (Theorems 7.1, 7.2 and 7.4), the proofs in 5] will now carry over to our context with only minor changes needed.

\subsection{Existence of the model structure}

Our goal in this first section is to establish Proposition 8.8, which abstractly builds the desired model structure on $\mathrm{dSet}^{G}$ by generalizing [5, Prop. 3.12].

As it turns out, adapting the treatment in [5, §3] requires only minimal modifications (essentially "adding $G$ to the statements therein"). As such, we will be brief in our discussion, and refer the reader to [5, §3] for extra details.

Recall the notation $J=N(0 \rightleftarrows 1)$ for the nerve of the groupoid generated by a single isomorphism between two distinct objects. As in [5, §3.2] we write $J_{d}=i_{!}(J)$ when regarding $J$ as a dendroidal set, and we will further regard $J_{d}$ as a $G$-dendroidal set by equipping it with the trivial $G$-action.

Following [5, §3.2], we define An, the class of $J$-anodyne extensions, to be the saturation of the $G$-inner horn inclusions together with the maps

$$
\{i\} \otimes \Omega[T] \coprod_{\{i\} \otimes \partial \Omega[T]} J_{d} \otimes \partial \Omega[T] \rightarrow J_{d} \otimes \Omega[T], \quad i=0,1, \quad T \in \Omega_{G} .
$$

A $G$-dendroidal set $X$ (resp. map $X \rightarrow Y$ ) is then called $J$-fibrant (resp. $J$ fibration) if it has the right lifting property with respect to the maps in An.

The following generalizes the necessary parts of [5, Prop. 3.3] (note that that result is slightly corrected in the erratum to [5]).

Proposition 8.2. Let $A \rightarrow B$ be a normal monomorphism in $\mathrm{dSet}^{G}$. Then:

(i) for $i=0,1$ the map $\{i\} \otimes B \amalg_{\{i\} \otimes A} J_{d} \otimes A \rightarrow J_{d} \otimes B$ is in An;

(ii) $\{0,1\} \otimes B \amalg_{\{0,1\} \otimes A} J_{d} \otimes A \rightarrow J_{d} \otimes B$ is a normal monomorphism, which is An if $A \rightarrow B$ is.

Proof. (i) follows since normal monomorphisms are built by attaching boundary inclusions $\partial \Omega[T] \rightarrow \Omega[T]$ for $T \in \Omega_{G}$. Similarly, (ii) follows from the " $S$ linear" cases of Proposition 7.25 and Theorem 7.1 (recall that $J_{d}$ is a simplicial set). 
As in [5, §3.4], for a $G$-dendroidal set $B$ we now write $\mathrm{dSet}^{G} / B$ for the category of $G$-dendroidal sets over $B$ and let $\operatorname{An}_{B}$ denote the class of maps in $\mathrm{dSet}^{G} / B$ whose image in $\mathrm{dSet}^{G}$ is in An.

Proposition 8.3. Write $\partial^{0}:\{0\} \rightarrow J_{d}, \partial^{1}:\{1\} \rightarrow J_{d}, \sigma: J_{d} \rightarrow\{*\}$ for the standard map $\S^{3}$ and abbreviate $\mathcal{J}=\left(J_{d} \otimes-, \partial^{0} \otimes-, \partial^{1} \otimes-, \sigma \otimes-\right)$.

Then whenever $B$ is normal the pair $\left(\mathcal{J}, \mathrm{An}_{B}\right)$ is a homotopical structure on $\mathrm{dSet}^{G} / B$ as defined in [4, Def. 1.3.14].

Proof. There are two parts: showing that $\mathcal{J}$ is an elementary homotopical datum as in [4, Def. 1.3.6] and that $\mathrm{An}_{B}$ is a class of anodyne extensions with respect to $\mathcal{J}$ as in [4, Def. 1.3.10]. We will refer to the axiom names used therein.

For the first claim, both axioms DH1 and DH2 follow from Proposition 7.25 since when $B$ is normal all monomorphisms over $B$ are normal monomorphisms.

For the second claim, axiom An0 follows by [4, Lemma 1.3.52] while An1,An2 follow from Proposition 8.2

Combining 4, Thm. 1.3.22, Prop. 1.3.31, Prop. 1.3.36, Lemma 1.3.52] now yields the following (this generalizes [5, Prop. 3.5, Remark 3.6]).

Proposition 8.4. For any normal $G$-dendroidal set $B$, the category $\mathrm{dSet}^{G} / B$ has a left proper cofibrantly generated model structure such that

- the cofibrations are the monomorphisms;

- J-anodyne extensions over $B$ are trivial cofibrations;

- the fibrant objects are the $X \stackrel{p}{\rightarrow} B$ such that $p$ is a J-fibration in $\mathrm{dSet}^{G}$;

- a map $X \stackrel{f}{\rightarrow} X^{\prime}$ between fibrant $X \rightarrow B, X^{\prime} \rightarrow B$ is a fibration in $\mathrm{dSet}^{G} / B$ iff $f$ is a $J$-fibration in $\mathrm{dSet}^{G}$.

The following generalizes [5, Lemma 3.7] again following from [4, Cor. 1.3.35].

Lemma 8.5. Let $X \rightarrow Y$ be a trivial fibration between normal $G$-dendroidal sets. Then any section $s: Y \rightarrow X$ is in An.

Fix once and for all a normalization $E_{\infty}$ of the terminal $G$-dendroidal set $*$, i.e. a trivial fibration $E_{\infty} \rightarrow *$ with $E_{\infty}$ a normal $G$-dendroidal set.

The following generalizes [5] Lemma 3.9].

Lemma 8.6. For any normal G-dendroidal set $X$ and map $a: X \rightarrow E_{\infty}$, the $\operatorname{map}(a, i d): X \rightarrow E_{\infty} \times X$ is in An.

Proof. Since $(a, i d)$ is a section of the projection $X \times E_{\infty} \rightarrow X$, this follows from Lemma 8.5.

The following generalizes [5, Lemma 3.10].

Lemma 8.7. Let $i: A \rightarrow B$ be a map of normal $G$-dendroidal sets and $p: X \rightarrow Y$ any map of $G$-dendroidal sets. Then $p$ has the right lifting property with respect to $i$ iff, for any map $B \rightarrow E_{\infty}$, the map $E_{\infty} \times X \stackrel{i d \times p}{\longrightarrow} E_{\infty} \times Y$ has the right lifting property with respect to $i$ in $\mathrm{dSet}^{G} / E_{\infty}$.

\footnotetext{
${ }^{3}$ Note that $\{*\}=i_{!}(*)$ denotes the terminal simplicial set, not the terminal dendroidal set.
} 
Proof. Given a lifting problem as on the left below, one obtains a lifting problem as on the right by arbitrarily choosing a map $B \rightarrow E_{\infty}$ (such a map always exists since $B$ is assumed normal). It is clear that the lifting problems are equivalent.
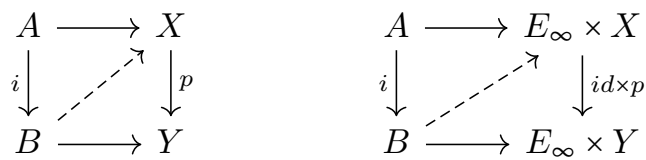

We finally obtain the model structure on $\mathrm{dSet}^{G}$, generalizing [5, Prop. 3.12].

Proposition 8.8. $\mathrm{dSet}^{G}$ is equipped with a left proper cofibrantly generated model structure such that

- the cofibrations are the normal monomorphisms;

- inner G-anodyne extensions are trivial cofibrations;

- the fibrant objects are the J-fibrant objects;

- the fibrations between fibrant objects are the J-fibrations.

Proof. As in [5, Prop. 3.12], the model structure is built via the adjunction

$$
p_{!}: \mathrm{dSet}^{G} / E_{\infty} \rightleftarrows \mathrm{dSet}^{G}: p^{*}
$$

where $p_{!}(X \rightarrow B)=X, p^{*}(X)=\left(E_{\infty} \times X \rightarrow E_{\infty}\right)$. Noting that both $p_{!}$and $p^{*}$ preserve all colimits it follows that condition (iii) in [4, Prop. 1.4.23] reduces to verifying that $p^{*} p_{!}(j)$ is a trivial cofibration in $\mathrm{dSet}^{G} / E_{\infty}$ whenever $j$ is. Since this follows from Lemma 8.6. the transfer model structure exists.

The claim that cofibrations are normal monomorphisms follows since all monomorphisms over $E_{\infty}$ are normal. The converse follows since all boundary inclusions $\partial \Omega[T] \rightarrow \Omega[T]$ are in the image of $p_{!}$, and the claim concerning anodyne extensions follows by the same argument. The fibrancy claims follow from Lemma 8.7. Lastly, left properness follows from that in Proposition 8.4 together with the observation that $p^{*}$ preserves both cofibrations and colimits and detects weak equivalences.

Remark 8.9. As in the case of the model structure on dSet or of the Joyal model structure on sSet, the trivial cofibrations (resp. fibrations) in Proposition 8.8 do not coincide with the inner $G$-anodyne extensions (resp. $J$-fibrations), but merely contain (resp. are contained in) them.

\subsection{Characterization of fibrant objects}

Much as in [5], the bulk of the work is now that of characterizing the fibrant objects as indeed being the $G$ - $\infty$-operads.

We will need to make use of the adjunction

$$
u^{*}: \mathrm{dSet}_{G} \rightleftarrows \mathrm{dSet}^{G}: u_{*}
$$

discussed in Proposition 5.53 . 
Definition 8.10. The class of normal monomorphims of $\mathrm{dSet}_{G}$ is the saturation of the maps of the form $u_{*}(A \rightarrow B)$ for $A \rightarrow B$ a normal monomorphism in $\mathrm{dSet}^{G}$. Further, a map $X \rightarrow Y$ in $\mathrm{dSet}_{G}$ is called a trivial fibration if it has the right lifting property with respect to normal monomorphisms.

We now extend some notation from [5, §6.1].

As usual, for $X$ an $\infty$-category, $k(X)$ will denote the maximal Kan complex inside $X$ and $\tau(X) \in$ Cat will denote its homotopy category.

Notation 8.11. For a $G$ - $\infty$-operad $X$ and simplicial set $K$ (thought of as having a trivial $G$-action), we define $X^{(K)} \in \operatorname{dSet}_{G}$ to have $T$-dendrices (recall $\left.T \in \Omega_{G}\right)$ the maps

$$
i_{!}(K) \otimes \Omega[T] \stackrel{a}{\rightarrow} X
$$

such that for each edge orbit $G / H \cdot \eta \stackrel{G / H \cdot e}{\longrightarrow} T$ the induced map

$$
K \stackrel{a_{e}}{\longrightarrow} i^{*}\left(X^{H}\right)
$$

factors through $k\left(i^{*}\left(X^{H}\right)\right)=k\left(\left(i^{*}(X)\right)^{H}\right)$.

Remark 8.12. Note that for $X$ a $G$ - $\infty$-category one always has $k\left(X^{G}\right) \subset$ $k(X)^{G}$, but that this inclusion is rarely an equality.

Notation 8.13. For a normal $G$-dendroidal set $A \in \mathrm{dSet}^{G}$ and a $G$ - $\infty$-operad $X$ we define $k(A, X) \in \mathrm{sSet}$ to have $n$-simplices the maps

$$
i_{!}(\Delta[n]) \otimes A \stackrel{b}{\rightarrow} X
$$

such that, for all element orbits $G / H \cdot \eta \stackrel{G / H \cdot a}{\longrightarrow} A$ the induced map

$$
\Delta[n] \stackrel{b_{a}}{\longrightarrow} i^{*}\left(X^{H}\right)
$$

factors through $k\left(i^{*}\left(X^{H}\right)\right)=k\left(\left(i^{*}(X)\right)^{H}\right)$.

Note that there are canonical isomorphisms

$$
H_{o m} \mathrm{sSet}(K, k(A, X)) \simeq \operatorname{Hom}_{\mathrm{dSet}_{G}}\left(u_{*}(A), X^{(K)}\right) .
$$

The following is the analogue of [5, Thm. 6.4]. Recall that a map $\mathcal{C} \rightarrow \mathcal{D}$ in Cat is called a categorical fibration if it has the right lifting property against the inclusion $1 \rightarrow[1]$, where $[1]=(0 \rightarrow 1)$.

Theorem 8.15. Let $p: X \rightarrow Y$ be a $G$-inner fibration between $G$ - $\infty$-operads. Then

$$
e v_{1}: X^{(\Delta[1])} \rightarrow Y^{(\Delta[1])} \times_{u_{*}(Y)} u_{*}(X)
$$

has the right lifting property with respect to inclusions $u_{*}(\partial \Omega[S] \rightarrow \Omega[S])$ for any $G$-tree $S \in \Omega_{G}$ with at least one vertex.

Consequently, (8.16) is a trivial fibration in $\mathrm{dSet}_{G}$ iff all maps $\tau i^{*}\left(X^{H} \rightarrow Y^{H}\right)$ for $H \leq G$ are categorical fibrations. 
Proof. Noting that it is $S \simeq G \cdot{ }_{H} S_{e}$ for some $S_{e} \in \Omega^{H}, H \leq G$, it suffices to deal with the case $S \in \Omega^{G}$.

The proof of the main claim now follows exactly as in the proof of [5, Thm. 6.4] by replacing uses of [5, Thm. 5.2] and [5, Thm. 4.2] with the equivariant analogues Theorems 7.2 and 7.4 .

For the "consequently" part, one needs only note that in the equivariant context there are now multiple $G$-trees with no vertices, namely the $G$-trees of the form $G / H \cdot \eta$.

The following is the analogue of [5, Prop. 6.7].

Proposition 8.17. Let $p: X \rightarrow Y$ be a $G$-inner fibration between $G$ - $\infty$-operads. If $\tau i^{*}\left(X^{H} \rightarrow Y^{H}\right)$ is a categorical fibration for all $H \leq G$ then, for any monomorphism between normal dendroidal sets $A \rightarrow B$, the map

$$
k(B, X) \rightarrow k(B, Y) \times_{k(A, Y)} k(A, X)
$$

is a Kan fibration between Kan complexes.

Proof. We will mainly refer to the proof of [5, Prop. 6.7] while indicating the main changes. First, note that it follows from Theorem [7.1(ii) that the map $\underline{\operatorname{Hom}}(B, X) \rightarrow \underline{\operatorname{Hom}}(B, Y) \times_{\underline{H o m}(A, Y) \underline{H o m}}(A, X)$ of simplicial mapping spaces is a $G$-inner fibration between $G$ - $\infty$-categories, and thus so is (8.18). As in [5, Prop. 6.7], it now suffices to check that (8.18) has the right lifting property against the "left pushout products"

$$
\partial \Delta[n] \times \Delta[1] \cup_{\partial \Delta[n] \times\{1\}} \Delta[n] \times\{1\} \rightarrow \Delta[n] \times \Delta[1]
$$

(i.e. thanks to [5, Lemma 6.5] one needs only consider the case $i=1$ of (8.10). A lifting problem

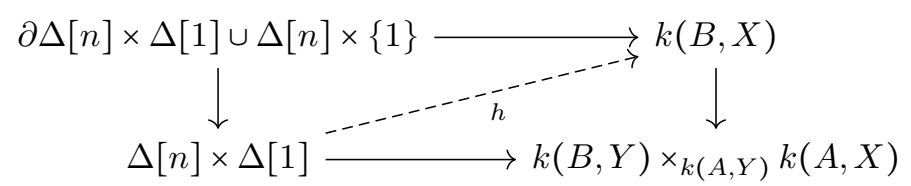

induces a (a priori non equivalent) lifting problem

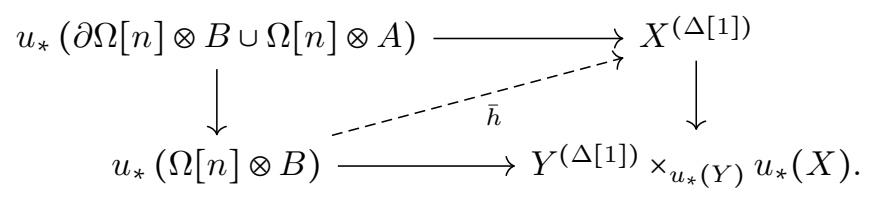

That the lift $\bar{h}$ in (8.20) exists follows from Theorem 8.15 and it hence remains to check that the adjoint map $i_{!}(\Delta[n] \times \Delta[1]) \otimes B \rightarrow X$ indeed provides the map $h$ in (8.19). I.e., one must check that for any element orbit $G / H \cdot b$ of $B$ the induced map $\Delta[n] \times \Delta[1] \rightarrow i^{*}\left(X^{H}\right)$ factors through $k\left(i^{*}\left(X^{H}\right)\right.$ ) (note that the existence of $\bar{h}$ only guarantees such a factorization for the restriction along $\{0,1, \cdots, n\} \times \Delta[1] \subset \Delta[n] \times \Delta[1])$. The $n=0$ case is immediate and the $n>0$ case 
follows by arguing using the "2-out-of-3 property", just as in the penultimate paragraph of the proof of [5, Prop. 6.7].

Standard arguments (setting $A=\varnothing$ of $Y=*$ just as in [5, Prop. 6.7] or as at the end of the proof of Theorem (7.4) finish the proof.

Combining the previous result with (8.14) now yields the following.

Corollary 8.21. Let $p: X \rightarrow Y$ be a $G$-inner fibration between $G$ - $\infty$-operads such that $\tau i^{*}\left(X^{H} \rightarrow Y^{H}\right)$ is a categorical fibration for all $H \leq G$. Then, for any anodyne extension of simplicial sets $K \rightarrow L$,

$$
X^{(L)} \rightarrow Y^{(L)} \times_{Y^{(K)}} X^{(K)}
$$

is a trivial fibration in $\mathrm{dSet}_{G}$.

We now obtain our sought generalization of [5, Thm. 6.10].

Theorem 8.22. A G-dendroidal set $X$ is $J$-fibrant iff it is a $G$ - $\infty$-operad. Further, a $G$-inner fibration $p: X \rightarrow Y$ between $G$ - $\infty$-operads is a $J$-fibration iff $\tau i^{*}\left(X^{H} \rightarrow Y^{H}\right)$ is a categorical fibration for all $H \leq G$.

Proof. It suffices to prove the "further" claim. Moreover, the "only if" direction is a direct consequence of [12, Cor. 1.6]. Unwinding definitions and adjunction properties it thus remains to show that

$$
X^{J_{d}} \rightarrow Y^{J_{d}} \times_{Y^{\{0\}}} X^{\{0\}}
$$

is a trivial fibration in $\mathrm{dSet}^{G}$ if $\tau i^{*}\left(X^{H} \rightarrow Y^{H}\right)$ is a categorical fibration for all $H \leq G$. We now note that since any map $J_{d} \rightarrow Z$ necessarily factors through $k(Z)$ the map

$$
u_{*}\left(X^{J_{d}} \rightarrow Y^{J_{d}} \times_{Y^{\{0\}}} X^{\{0\}}\right)
$$

coincides with the map

$$
X^{\left(J_{d}\right)} \rightarrow Y^{\left(J_{d}\right)} \times_{Y(\{0\})} X^{(\{0\})}
$$

which is a trivial fibration in $\mathrm{dSet}_{G}$ by Corollary 8.21. The result now follows since $\mathrm{dSet}^{G}$ is a reflexive subcategory of $\mathrm{dSet}_{G}$, so that $u_{*}(f)$ is a trivial fibration iff $f$ is.

The following follows exactly as in [5, Cor. 6.11].

Corollary 8.23. The weak equivalences in $\mathrm{dSet}^{G}$ are the smallest class containing the inner $G$-anodyne extensions, the trivial fibrations and closed under "2-out-of-3".

\section{Indexing system analogue results}

In this section we follow the lead of 2] and build variant model structures on $\mathrm{dSet}^{G}$ associated to indexing systems, a notion originally introduced in 2, Def. 3.22 , which we repackage (and slightly extend) in Definition 9.5.

Definition 9.1. A $G$-graph subgroup of $G \times \Sigma_{n}$ is a subgroup $K \leq G \times \Sigma_{n}$ such that $K \cap \Sigma_{n}=*$. 
Remark 9.2. $G$-graph subgroups are graphs of homomorphisms $G \geq H \rightarrow \Sigma_{n}$.

Definition 9.3. A G-vertex family is a collection

$$
\mathcal{F}=\coprod_{n \geq 0} \mathcal{F}_{n}
$$

where each $\mathcal{F}_{n}$ is a family of $G$-graph subgroups of $G \times \Sigma_{n}$ closed under subgroups and conjugation.

Further, a $H$-set $X$ for a subgroup $H \leq G$ is called a $\mathcal{F}$-set if for some (and hence any) choice of isomorphism $X \simeq\{1, \cdots, n\}$ the graph subgroup of $G \times \Sigma_{n}$ encoding the $H$-action on $\{1, \cdots, n\}$ is in $\mathcal{F}$.

Definition 9.4. Let $\mathcal{F}$ be a $G$-vertex family. A $G$-tree $T$ is called a $\mathcal{F}$-tree if for all edges $e \in T$ with isotropy $H$ one has that the $H$-set $e^{\uparrow}$ is a $\mathcal{F}$-set.

It is clear that whenever $T \rightarrow S$ is either an outer face or a quotient, $S$ being a $\mathcal{F}$-tree implies that so is $T$. However, the same is typically not true for inner faces and degeneracies.

Definition 9.5. A $G$-vertex family $\mathcal{F}$ is called a weak indexing system if $\mathcal{F}$ trees form a sieve of $\Omega_{G}$, i.e. if for any map $T \rightarrow S$ with $S$ a $\mathcal{F}$-tree then it is also $T$ a $\mathcal{F}$-tree. In this case we denote the sieve of $\mathcal{F}$-trees by $\Omega_{\mathcal{F}} \subset \Omega_{G}$.

Additionally, $\mathcal{F}$ is called an indexing system if every $\mathcal{F}_{n}$ contains all subgroups $H \times * \leq G \times \Sigma_{n}$ for $H \leq G, n \geq 0$.

Remark 9.6. Closure under degeneracies is simply the statement that $\mathcal{F}_{1}$ contains all subgroups $H=H \times \Sigma_{1} \leq G \times \Sigma_{1}$ for $H \leq G$.

Remark 9.7. Since Definition 9.5 may at first seem to be quite different from the original [2, Def. 3.22], we now address the equivalence between the two. To a $H$-set with orbital decomposition $H / K_{1} \sqcup \cdots \sqcup H / K_{n}$ one can associate the $G$-corolla with orbital representation as follows.

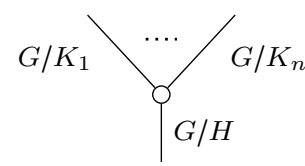

Note that for any of its roots $r$ one has that $r^{\uparrow}$ is a $G$-conjugate of the $H$-set $H / K_{1} \amalg \cdots \amalg H / K_{n}$. The conditions (cf. [2, Def. 3.22]) that indexing systems are closed under disjoint unions [2, Def. 3.19] and sub-objects [2, Def. 3.21] of $\mathcal{F}$-sets are then encoded by taking inner faces of $\mathcal{F}$-trees of the form
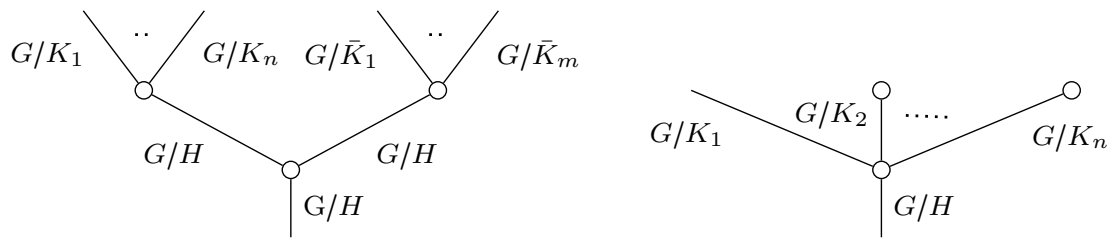
while the closure under self-induction [2, Def. 3.20] is similarly encoded by $\mathcal{F}$-trees as on the left below.
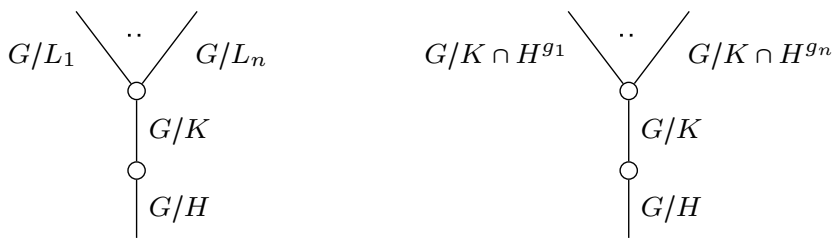

Closure under cartesian products 2, Def. 3.22] is in fact redundant, as the double coset formula $H / K \times H / L \simeq \amalg_{[g] \in L \backslash H / K} H / K \cap L^{g}$ allows such $H$-sets to be built using self inductions as displayed by the rightmost tree above (the case of products of sets with multiple orbits being then obtained via disjoint units).

Definitions 6.3, 6.11 and 6.12 admit weak indexing system analogues.

Definition 9.8. Let $\mathcal{F}$ be a weak indexing system.

A $\mathcal{F}$-boundary inclusion (resp. $\mathcal{F}$-inner horn inclusion) is a boundary inclusion $\partial \Omega[T] \rightarrow \Omega[T]$ (resp. inner horn inclusion $\Lambda^{G e}[T] \rightarrow \Omega[T]$ ) with $T \in \Omega_{\mathcal{F}}$.

A monomorphism is called $\mathcal{F}$-normal (resp. $\mathcal{F}$-anodyne) if it is in the saturation of $\mathcal{F}$-boundary inclusions (resp. $\mathcal{F}$-inner horn inclusions) under pushouts, transfinite compositions and retracts.

Finally, a $G$-dendroidal set $X$ is called a $\mathcal{F}$ - $\infty$-operad if it has the right lifting property with respect to all $\mathcal{F}$-inner horn inclusions.

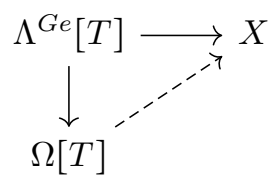

We now list the necessary modifications to extend the results in this paper to the indexing system case.

A direct analogue of Proposition 6.5 yields that $X \in \mathrm{dSet}^{G}$ is $\mathcal{F}$-normal (i.e. $\varnothing \rightarrow X$ is a $\mathcal{F}$-normal monomorphism) iff all dendrices $x \in X(T)$ have $\mathcal{F}$-isotropy, i.e. isotropies $\Gamma \leq G \times \Sigma_{T}$ that are graph subgroups for partial homomorphisms $G \geq H \rightarrow \Sigma_{T}$ such that the induced $G$-tree $G \cdot H T$ is a $\mathcal{F}$-tree.

It then follows that, much like normal dendroidal sets, $\mathcal{F}$-normal dendroidal sets form a sieve, i.e., for any map $X \rightarrow Y$ with $Y$ a $\mathcal{F}$-normal dendroidal set then so is $X$.

Noting that the subtrees of $S \otimes T$ are $\mathcal{F}$-trees whenever $S, T$ are $\mathcal{F}$-trees (since the generating vertices/broad relations of $S \otimes T$ are induced from those of $S, T)$, it follows that $\Omega[S] \otimes \Omega[T]$ is then $\mathcal{F}$-normal so that the sieve condition implies that Proposition 7.25 generalizes to the $\mathcal{F}$-normal case.

Likewise, the key results Theorem 7.1 and 7.2 immediately generalize by replacing the terms " $G$-tree" and " $G$-anodyne" with " $\mathcal{F}$-tree" and " $\mathcal{F}$-anodyne". This is because their proofs, while long, ultimately amount to identifying suitable edge orbits of suitable subtrees of $S \otimes T$ and then attaching the corresponding equivariant horns.

Likewise, Proposition 7.53 generalizes to the $\mathcal{F}$ case for the same reason, and hence so does Theorem 7.4, since its proof is an application of Proposition 7.53.

We can now prove Theorem 2.2 . 
proof of Theorem 2.2. The proof of the existence of the model structure follows just as in 88.1 . The only notable changes are as follows: in defining $J_{\mathcal{F}}$-anodyne extensions one uses only $\mathcal{F}$-inner horns and those maps in (8.1) for $T \in \Omega_{\mathcal{F}}$; the term "normal" is replaced with " $\mathcal{F}$-normal" throughout (note that any monomorphism over a $\mathcal{F}$-normal dendroidal set is a $\mathcal{F}$-normal monomorphism).

The characterization of the $J_{\mathcal{F}}$-fibrant objects as being the $\mathcal{F}$ - $\infty$-operads follows by repeating the arguments in 8.2 though some care is needed when adapting the definitions preceding Theorem 8.15 Firstly, letting $\Omega_{\mathcal{F}} \subset \Omega_{G}$ denote the sieve of $\mathcal{F}$-trees, one sets $\mathrm{dSet}_{\mathcal{F}}=\mathrm{dSet}^{\Omega_{\mathcal{F}}^{o p}}$, leading to an adjunction

$$
u^{*}: \mathrm{dSet}_{\mathcal{F}} \leftrightarrows \mathrm{dSet}^{G}: u_{*}
$$

allowing for the $\mathcal{F}$-normal monomorphisms of $\mathrm{dSet}_{\mathcal{F}}$ to be defined from the $\mathcal{F}$-normal monomorphisms in $\mathrm{dSet}^{G}$ just as in Definition 8.10,

For a $\mathcal{F}$ - $\infty$-operad and simplicial set $K$, one defines $X^{(K)} \in \mathrm{dSet}_{\mathcal{F}}$ just as in Notation 8.11 while for $A \in \mathrm{dSet}^{G}$ a $\mathcal{F}$-normal dendroidal set and $\mathcal{F}$ - - -operad $\mathrm{X}$ one defines $k(A, X) \in \mathrm{s}$ Set just as in Notation 8.13.

The proofs of Theorems 8.15 Proposition 8.17 and Theorem 8.22 now extend mutatis mutandis by using the $\mathcal{F}$ versions of Theorems 7.2 and 7.4 .

\section{References}

[1] C. Berger and I. Moerdijk. On an extension of the notion of Reedy category. Math. Z., 269(3-4):977-1004, 2011.

[2] A. J. Blumberg and M. A. Hill. Operadic multiplications in equivariant spectra, norms, and transfers. Adv. Math., 285:658-708, 2015.

[3] P. Bonventre and L. A. Pereira. Genuine equivariant operads. arXiv preprint: 1707.02226, 2017.

[4] D.-C. Cisinski. Les préfaisceaux comme modèles des types d'homotopie. Astérisque, (308):xxiv+390, 2006.

[5] D.-C. Cisinski and I. Moerdijk. Dendroidal sets as models for homotopy operads. J. Topol., 4(2):257-299, 2011.

[6] D.-C. Cisinski and I. Moerdijk. Dendroidal Segal spaces and $\infty$-operads. J. Topol., 6(3):675-704, 2013.

[7] D.-C. Cisinski and I. Moerdijk. Dendroidal sets and simplicial operads. J. Topol., 6(3):705-756, 2013.

[8] D.-C. Cisinski and I. Moerdijk. Note on the tensor product of dendroidal sets. Available at: https://arxiv.org/abs/1403.6507, 2014.

[9] S. R. Costenoble and S. Waner. Fixed set systems of equivariant infinite loop spaces. Trans. Amer. Math. Soc., 326(2):485-505, 1991.

[10] E. Getzler and J. Jones. Operads, homotopy algebra and iterated integrals. Available at: https://arxiv.org/abs/hep-th/9403055, 1995. 
[11] G. Heuts, V. Hinich, and I. Moerdijk. On the equivalence between Lurie's model and the dendroidal model for infinity-operads. Adv. Math., 302:8691043, 2016.

[12] A. Joyal. Quasi-categories and Kan complexes. J. Pure Appl. Algebra, 175(1-3):207-222, 2002. Special volume celebrating the 70th birthday of Professor Max Kelly.

[13] J. Lurie. Higher topos theory, volume 170 of Annals of Mathematics Studies. Princeton University Press, Princeton, NJ, 2009.

[14] M. Markl, S. Shnider, and J. Stasheff. Operads in algebra, topology and physics, volume 96 of Mathematical Surveys and Monographs. American Mathematical Society, Providence, RI, 2002.

[15] J. P. May. The geometry of iterated loop spaces. Springer-Verlag, BerlinNew York, 1972. Lectures Notes in Mathematics, Vol. 271.

[16] I. Moerdijk and I. Weiss. Dendroidal sets. Algebr. Geom. Topol., 7:14411470, 2007.

[17] I. Moerdijk and I. Weiss. On inner Kan complexes in the category of dendroidal sets. Adv. Math., 221(2):343-389, 2009.

[18] M. Stephan. On equivariant homotopy theory for model categories. Homology Homotopy Appl., 18(2):183-208, 2016.

[19] I. Weiss. Broad posets, trees, and the dendroidal category. Available at: https://arxiv.org/abs/1201.3987, 2012. 